\author{
Universidade de São Paulo \\ Instituto de Física
}

\title{
Sobre o estado fundamental de teorias de $n$-gauge abelianas topológicas
}

\author{
Javier Ignacio Lorca Espiro
}

Orientador: Prof. Dr. Paulo Teotônio-Sobrinho

Tese de doutorado apresentada ao Instituto de Física da Universidade de São Paulo, como requisito parcial para a obtenção do título de Doutor em Ciências.

Banca Examinadora:

Prof. Dr. Paulo Teotônio-Sobrinho - Orientador (IFUSP)

Prof. Dr. Fernando Tadeu Caldeira Brandt (IFUP)

Prof. Dr. Luis Gregório Godoy de Vasconcellos Dias da Silva (IFUSP)

Prof. Dr. Eliezer Batista (UFSC)

Prof. Dr. Eduardo Peres Novais de Sá (UFABC) 


\section{FICHA CATALOGRÁFICA \\ Preparada pelo Serviço de Biblioteca e Informação do Instituto de Física da Universidade de São Paulo}

\section{Lorca Espiro, Javier Ignacio}

Sobre o estado fundamental de teorias de calibre abelianas topológicas. São Paulo, 2017.

Tese (Doutorado) - Universidade de São Paulo. Instituto de Física. Depto. de Física Matemática

Orientador(a): Prof. Dr. Paulo Teotônio-Sobrinho Área de Concentração: Física Matemática.

Unitermos: 1. Topologia algébrica; 2. Teoria de calibre; 3. Cohomologia; 4. Homologia; 5. Mecânica quântica. 


\title{
University of São Paulo \\ Physics Institute
}

\section{On the ground state of abelian topological higher gauge theories}

\author{
Javier Ignacio Lorca Espiro
}

Supervisor: Prof. Dr. Paulo Teotônio-Sobrinho

Thesis submitted to the Physics Institute of the University of So Paulo in partial fulfillment of the requirements for the degree of Doctor of Science.

Examining Committee:

Prof. Dr. Paulo Teotônio-Sobrinho - Supervisor (IFUSP)

Prof. Dr. Fernando Tadeu Caldeira Brandt (IFUP)

Prof. Dr. Luis Gregório Godoy de Vasconcellos Dias da Silva (IFUSP)

Prof. Dr. Eliezer Batista (UFSC)

Prof. Dr. Eduardo Peres Novais de Sá (UFABC) 


\section{On the ground state of abelian topological higher gauge theories}

Author:

J. Lorca EsPIRO
Supervisor:

Dr. Paulo Teotônio Sobrinho

A document submitted in fulfilment of the requirements

for the degree of Doctor of Philosophy

FÍSICA TEÓRICA

Departmento de Física Matemática

June 2017 
"What they figured out (scientists), is one particular response to their actions, and this response gives this universe, and the reality that is behind this is laughing! 'Ha ha! They think they have found me out!"

Paul Feyerabend 
UNIVERSIDADE DE SÃO PAULO

\author{
Abstract \\ IFUSP \\ Departmento de Física Matemática \\ Doctor of Philosophy
}

On the ground state of abelian topological higher gauge theories

by J. Lorca EsPIRO

The following is a document that aims to fulfill the requirements to be eligible as a $\mathrm{Ph} . \mathrm{D}$. in the Física Matemática Department of the University of Sáo Paulo (USP) 


\section{Acknowledgements}

Many thanks to my family, Jorge, Isabel and Constanza, for the unconditional support and encouragement during this five years time. I thank my girlfriend Marie-Carmelle for the uttermost support and love during these last two (and difficult) of the Doctorate. I also specially thank my advisor, Professor Dr. Paulo Teotônio Sobrinhoand the research group lead by him for the time and knowledge. And finally, I thank my partners in crime in this research, for a fun journey in the depths of mathematical physics: Pablo Ibieta Jimenez and Ricardo Almeida. 


\section{Contents}

$\begin{array}{ll}\text { Abstract } & \text { ii }\end{array}$

$\begin{array}{ll}\text { Acknowledgements } & \text { iii }\end{array}$

List of Figures vi vi v v

List of Tables $\quad$ ix

1 Introduction 1

1.1 Topological order . . . . . . . . . . . . . . . . . . . . . . 1

1.2 Characterization and classification .............. 3

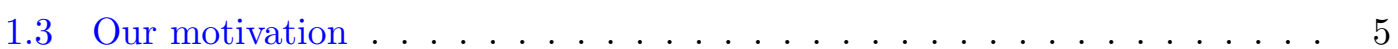

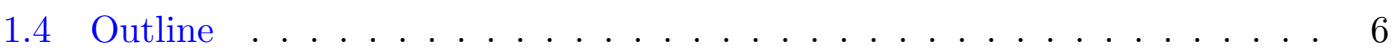

2 Gauge Theory Fundamentals $\quad \mathbf{8}$

2.1 Brief review of the differentio-geometric approach to gauge theories . . . . 9

2.2 Traditional approach to gauge theory . . . . . . . . . . . . . . . . 12

2.3 The categorical view of gauge theories . . . . . . . . . . . 16

2.3 .1 The 1 -gauge . . . . . . . . . . . . . . . 16

2.3 .2 The 2 -gauge. . . . . . . . . . . . . . . . . 20

2.4 Criticism of the $n$-group approach $\ldots \ldots \ldots \ldots \ldots$

3 The Toric Code and Quantum Double Models 27

3.1 Lattice gauge theory . . . . . . . . . . . . . . . . . . . . . 28

3.1.1 Observables, Wilson Loops and Gauge Fixing . . . . . . . . . . . . 31

3.2 Quantum Double models overview . . . . . . . . . . . . . . . . . 34

3.2 .1 The toric code . . . . . . . . . . . . . . 35

3.2 .2 The algebra: The Drinfeld double of a finite group . . . . . . . . 36

3.2.3 The quantum double model formalism . . . . . . . . . . . . . . . . . 39

3.2.4 Ribbon operators and elementary excitations . . . . . . . . . . 43

3.3 Examples of generalizations . . . . . . . . . . . . . . . . . 46

3.3.1 The "1-2 gauge" theory à la quantum double model . . . . . . . . 47

3.3.2 The "2-3 gauge" theory à la quantum double model . . . . . . . . 50

3.4 Comments . . . . . . . . . . . . . . . . . . . . 52 
4 Higher dimensional abelian gauge theories $\mathbf{5 4}$

4.1 Hilbert Space and Quantum States . . . . . . . . . . . . . . . . . . 55

4.1.1 Operators and Algebraic Relations . . . . . . . . . . . . 56

4.2 Dynamics . . . . . . . . . . . . . . . . . . . . 62

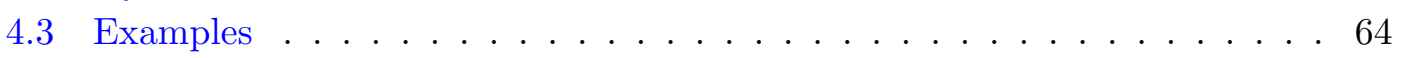

4.3.1 Abelian 1-gauge: Quantum Double Models . . . . . . . . . . . . 64

4.3.2 Abelian 1, 2-gauge theories . . . . . . . . . . . 67

5 Ground State Degeneracy for higher abelian gauge theories $\quad 68$

5.1 Projector Operators and Projection into $\mathcal{H}_{0} \ldots \ldots \ldots$. . . . . . . . . . . 68

5.1 .1 Gauge Equivalence . . . . . . . . . . . . . . . . . 72

5.2 The Ground State Degeneracy Theorem . . . . . . . . . . . . . . . . . . . . . . . 72

5.3 Examples . . . . . . . . . . . . . . . . . . . . . 75

5.3 .1 GSD of the Toric code . . . . . . . . . . . . . . . 75

5.3 .2 GSD of the 3D Toric Code on $T^{3} \ldots \ldots \ldots \ldots$

5.3 .3 Abelian 1,2-gauge theory . . . . . . . . . . . 76

5.3.4 Ground state of abelian 2-gauge and crossed module of racks . . . 77

5.4 Characterization of the Ground States . . . . . . . . . . . . . . . . . 78

5.4 .1 Measurement operators for the space $\mathcal{H}_{0} \ldots \ldots \ldots$. . . . . 79

5.4 .2 A geometrical description of the ground states . . . . . . . . 80

6 Final remarks $\quad \mathbf{8 3}$

6.1 Baby steps towards the study of the excited states . . . . . . . . . . 83

6.2 Summing up . . . . . . . . . . . . . . . . 88

6.3 Future work . . . . . . . . . . . . . . . . . . . . . 89

A Basic Differential geometry and Simplicial Decomposition 91

A.1 Basics of general topology . . . . . . . . . . . . . . . . 91

A.2 Simplices and Simplicial Complexes . . . . . . . . . . . . . . . 98

$\begin{array}{lr}\text { B A brief account on Homology } & 102\end{array}$

B.1 Homology Groups . . . . . . . . . . . . . . . . . . . . . 102

B.2 Singular Homology . . . . . . . . . . . . . . . . . . . . . . 106

B.3 Chain complexes, exact sequences and relative homology . . . . . . . . . 107

$\begin{array}{lr}\text { C Topics in Algebraic Topology } & 114\end{array}$

C.1 The universal coefficient Theorem . . . . . . . . . . . . . . . . . . . . . . . 114

C.2 Cohomology with coefficients in a chain complex . . . . . . . . . 118 


\section{List of Figures}

2.1 Commuting bundle map . . . . . . . . . . . . . . . . . . . . . 9

2.2 A gauge transformation . . . . . . . . . . . . . . . . 11

2.3 Diagram of a global Gauge transformation for a $G$-bundle . . . . . . . . . 11

2.4 "Parallelepiped" over a bundle $P$ with negative curvature vs a "paralleliped" in a Euclidean space $E$ (null curvature), the dashed arrow represents the fiber in each case . . . . . . . . . . . . . 15

2.5 Composition of paths $\gamma: y \rightarrow z$ and $\delta: x \rightarrow y \ldots \ldots \ldots \ldots$

2.6 Homotopic paths in $X$ space between $x_{0}$ and $x_{1} \ldots \ldots \ldots \ldots$

2.7 Homotopic paths $\delta: y \rightarrow x$ and $\gamma: y \rightarrow x \ldots \ldots \ldots \ldots \ldots$

2.8 Composition of morphisms defining a group . . . . . . . . . . . 21

2.9 Horizontal composition of 2-morphisms . . . . . . . . . . . . . . . . . 22

2.10 Vertical composition of 2 -morphisms . . . . . . . . . . . . . 22

2.11 Group operation of horizontal composition of 2 -groups $\ldots . . . .23$

3.1 A section of a discretized 2- $d$ manifold into a lattice, Vertices $v_{i}$ are shown, as well as oriented edges $e_{i}$ and oriented faces $f_{i}$. . . . . . . . 29

3.2 parallel transport $g_{e}$ and gauge transformation $g_{e}{ }^{\prime}$ over an edge $e \ldots . .30$

3.3 Holonomy $g_{f}$ of a triangular face $f$. The directions of the edges $e_{i}$ are depicted by the blue arrows while the direction of the face is depicted by the red circular arrow. Note that the only direction which is inconsistent between the two is $e_{3}$, hence the inverse element is taken. . . . . . . . . 30

3.4 A fusion of vertices, the local operation depicted has absorbed the edge $e$ without changing the value of the partition function by setting its group element to the identity . . . . . . . . . . . . . . 33

3.5 A square ordered lattice, vertex $(v)$ and plaquette $(p)$ sites $\ldots \ldots . . .35$

3.6 Elementary excitations: charge $(\circ)$ living on the verteces, and flux $(\otimes)$ living on the plaquettes . . . . . . . . . . . . 36

3.7 Oriented link with proper basis element $|g\rangle$ associated to it, equivalence to its inverse element $\left|g^{-1}\right\rangle$ and the inversion of the link orientation . . . 39

3.8 Definition of the operators for a QDM, on a vertex $\left(A_{s}^{g}\right)$ and on a plaquette $\left(B_{s}^{h}\right)$. Note that if $h$ is in the center of $G, B_{s}^{h}$ depends only on the plaquette $p$ and not on the vertex $v \ldots \ldots$. . . . . . . . 40

3.9 Equivalence on a parallel transport for the left and right shift operators . 42

3.10 a) Reference cell for the action of the vertex operator $A_{s}^{g}$. b) Reference cell for the action of the a plaquette operator $B_{s}^{h}$ in terms of local shift and projector operators . . . . . . . . . . . . . 4 4 43

3.11 A ribbon $\xi$ on an oriented lattice $\mathcal{L}$, from $s_{0}=\left(v_{0}, p_{0}\right)$ to $s_{1}=\left(v_{1}, p_{1}\right) \ldots 44$

3.12 Definition of a ribbon operator $F_{\xi}^{h, g}$. $\xi$ is the ribbon connecting the sites

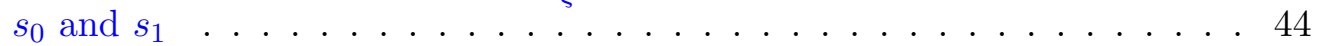


3.13 The commuting diagram of a Hopf Algebra . . . . . . . . . . . . . . . . 47

3.14 The right co-module . . . . . . . . . . . . . . . . 47

3.15 The right co-module . . . . . . . . . . . . . . . . . . . . . . . . . . . . . . . .

$3.16 \bar{A}_{v}^{g}, B_{p}^{h}$ and $C_{l}$ defined over local regions of $\mathcal{L}$, for $a, b, c, d, g, h, r, s, t, u, v \in$ $G_{1}$ and $\alpha, \beta, \gamma \in G_{0}$. Notice that $B_{p}^{h}$ is the same plaquette operator as the one defined for the QDM case. . . . . . . . . . . . . . . . 48

3.17 Section of an oriented lattice $\mathcal{L}$ that give support to the QDMv, where (i) the blue and green sectors have already been defined in Figure 4.4 and (ii) the new orange sector is associate to $l$-th shared link by two adjacent vertices containing discrete matter fields. . . . . . . . . . . . 49

3.18 Oriented lattice $\mathcal{L}$ (continuous) and its dual lattice $\mathcal{L}^{*}$ (dotted). . . . . . 50

3.19 Definition of the $A_{v}^{g}, \bar{B}_{p}^{h}$ and $D_{l}^{\tilde{\lambda}}$. Where $a, b, c, d, g \in G_{1}$ and $\tilde{\alpha}, \tilde{\beta}, \tilde{\gamma} \in$ $G_{2}$ with $a^{\prime}=f(\tilde{\lambda}) \cdot a, \tilde{\alpha}^{\prime}=\tilde{\alpha} * \tilde{\lambda}$ and $\tilde{\beta}^{\prime}=\tilde{\lambda}^{-1} * \tilde{\beta} \ldots \ldots . \ldots 51$

4.1 Diagrammatic description for $f \in \operatorname{hom}(C, G)_{0} \ldots \ldots \ldots 56$

4.2 A triangulated section of a 2-manifold . . . . . . . . . . . . . . . . 65

4.3 A regular squared section of a 2 -manifold . . . . . . . . . . . . . 65

4.4 Planar section of a oriented squared lattice $C(X)$ giving support to the QDM, with the pink and cyan sectors related to local the gauge $A_{g x^{*}}$ and local holonomy $B_{r y_{*}}$ operators, respectively. . . . . . . . . . 66

4.5 Definition of the local operators $A_{g x^{*}}$ and $B_{h y_{*}}$ with relation to the support regions depicted in FIG. 4.4. In this case $x \in K_{0}$ (i.e. vertices) and $y \in K_{2}$ (i.e. plaquetes) of a regular square lattice $C(X)$, with $a, b, c, d, g \in G_{1}$ and $h \in \hat{G}_{1} \ldots \ldots \ldots \ldots 6$

4.6 Definition of the local operators $A_{g x^{*}}$ and $B_{h y_{*}}$ with relation to the support regions depicted in FIG. 4.4. In this case $x \in K_{0}$ (i.e. vertices) and $y \in K_{2}$ (i.e. plaquetes) of a regular square lattice $C(X)$, with $a, b, c, d, g \in G_{1}$ and $h \in \hat{G}_{1} \ldots \ldots \ldots \ldots 6 \ldots \ldots \ldots \ldots \ldots$

5.1 Regular squared lattice embedded over a Torus $T^{2} \ldots \ldots \ldots$. . . . . . 75

5.2 hom $(C, G)_{0}$ for the toric code. . . . . . . . . . . . 75

5.3 Homology groups for the hom $(C, G)_{0}$ of the toric code. . . . . . . 76

5.4 hom $(C, G)_{0}$ for the abelian 1,2 -gauge theory. . . . . . . . . . 77

5.5 Homology groups for the abelian 1,2-gauge theory. . . . . . . . . . 77

5.6 A crossed module of racks . . . . . . . . . . . . . . . 78

5.7 A ground state of an abelian 2-gauge theory . . . . . . . . . . . 78

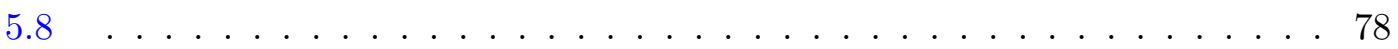

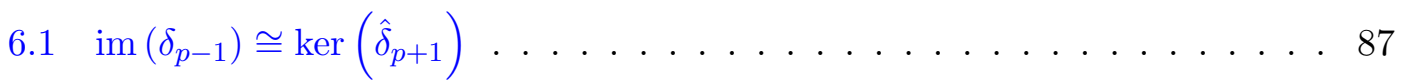

$6.2 \operatorname{im}\left(\hat{\delta}_{p+1}\right) \cong \operatorname{ker}\left(\delta_{p-1}\right) \ldots \ldots \ldots \ldots \ldots \ldots \ldots$

$6.3 \operatorname{im}\left(\hat{\delta}_{p+1}\right) \cong \operatorname{ker}\left(\hat{\delta}_{p}\right)$ and its dual $\operatorname{im}\left(\hat{\delta}_{p+1}\right) \cong \operatorname{ker}\left(\hat{\delta}_{p}\right) \ldots \ldots . . . .87$

$6.4 \operatorname{im} \widehat{\left(\delta_{p-1}\right)} \cong \operatorname{ker}\left(\delta_{p}\right)$ and its dual $\operatorname{im}\left(\delta_{p-1}\right) \cong \operatorname{ker}\left(\delta_{p}\right) \ldots \ldots . . \ldots 87$

A.1 Hausdorff axiom of separation, the open neighborhoods of $x\left(O_{x}\right)$ and $y$ $\left(O_{y}\right)$ do not intersect each other $\ldots \ldots \ldots . \ldots . \ldots . \ldots 2$ 
A.2 Open cover $M_{\alpha}$ go to an open subset $O_{\alpha}$ through the homeomorphic map

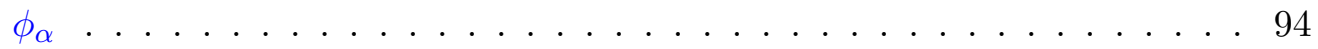

A.3 An orientable manifold (strip surface) . . . . . . . . . . . . . . . . 94

A.4 A non orientable manifold (a Möbius strip) . . . . . . . . . . . . . 94

A.5 Simplices in a $\mathbb{R}^{3}$ space . . . . . . . . . . . . . . . . . . . . 99

A.6 Schematic representation of a boundary operator acting over a 2-simplex 100

A.7 Triangulation of a closed annulus. It has 16 0-cells, 32 1-cells and 16 2-cells 101

B.1 a chain $\operatorname{map} \ldots \ldots \ldots \ldots$. . . . . . . . . . . . . . . . . . . . . . . . . . . . . . . .

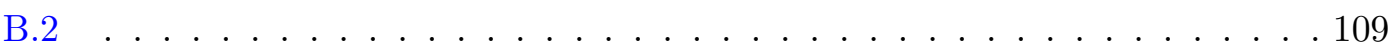

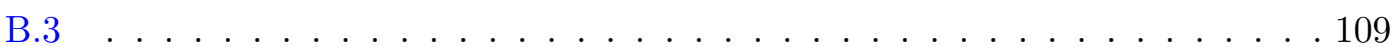

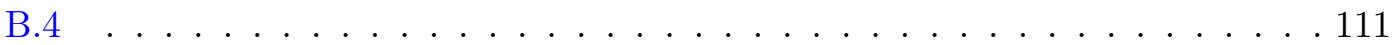

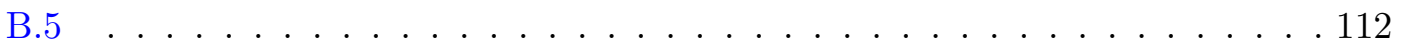

C.1 Diagrammatic description for $f \in \operatorname{hom}(C, G)_{0}$. Notice that the diagrams are not necessarily commutative. . . . . . . . . . . . 120 


\section{List of Tables}

3.1 Operations over a quantum double with $\mathbb{C}$-basis $\left\{g h^{*}: g, h \in G\right\} \ldots 37$

3.2 Local operators acting on a link $l, g, h \in G \ldots \ldots . \ldots . \ldots 42$ 
Dedicated to Jorge, Isabel, Constanza and Marie-Carmelle 


\section{Chapter 1}

\section{Introduction}

Throughout this work we embark on the two-fold task of giving an accessible and yet substantive account of discrete models with topological order, as well as to present our contributions to the field as part of the requirements for a Ph.D. degree at the University of São Paulo. We will first motivate the work which was undertaken by the group led by Dr. Paulo Teotônio-Sobrinho from a theoretical standpoint, and secondly, expose the promising results obtained as well as some of the perspectives for future works in this domain.

\section{$1.1 \quad$ Topological order}

The notions of symmetry and order have been central to understanding the mathematical structures of physical systems since the mid XIX century [1]. Symmetry has been typically included in physical theories by using ideas coming from group theory. These are usually included at the level of the action functional. During the XX century, quantum mechanics and later quantum field theory, were responsible for the spread of group theoretic techniques within the physics community. Ever since, this branch of mathematics has become an important part of the mathematical physics curricula. When a symmetry or correlation is observed on a many-body system, the term order is usually used. Furthermore, symmetry and order are typically characterized by a local order parameter. In opposition to this, we have sought in our work to understand the case in which there is an impossibility to find a local parameter to quantitatively describe a certain physical order. This fact is what naturally leads to the concept of topological order, which we will describe below.

To be more precise, orders can be broadly classified into two types [2]: 
- Global symmetry breaking orders. These are manifest in many physical systems. Its characterization is usually achieved by an explicit invariance of the basic interactions with respect to global symmetry operations. This is, when these are simultaneously performed on all of the fields in the system. At sufficiently low temperatures (or high coupling), such symmetries may often be spontaneously broken. These phenomena are mainly understood on the framework of the Landau's theory of phase transitions [3], through the use of local parameters and correlation functions [4].

It is worth mentioning, without going into details since it is out of the scope of this work, that symmetry breaking can be further classified into two types, these are explicit symmetry breaking and spontaneous symmetry breaking. Those are respectively distinguished according to whether the equations of motion fail to be invariant or the ground state fails to be invariant under these local symmetry operations.

- Topological orders. Studies about strongly correlated two-dimensional many-body systems show that there are theories where the mechanisms of spontaneous symmetry breaking and order parameters are not able to address the phase phenomena occurring at low temperatures. Even if global symmetry breaking is not taking place, the system can still exhibit what is known as topolgical order. This order may only be detected by non-local observables. The most important features of this new phase are a finite ground state degeneracy that depends on the topology of the system, as well as a phenomenon known as charge fractionalization (with respect to that of the constituent particles) and/or fractional statistics of the quasiparticles.

One of the first known examples of topological order, which has become some sort of an archetype for these systems, is the fractional quantum Hall (FQH) effect [5]. The electron filling factor for this system is $\nu=1 / n$ with $n$ odd. In this phase, the ground state is $n$-fold degenerate on a torus, while it is non-degenerate on a sphere, i.e. the ground state is dependent on the topology of the embedded space. In fact, the ground state degeneracy is responsible for the fractional quantization of Hall conductance and dictates the fractional charge and the exotic statistics of the quasiparticles found there [2]. We can also mention that bosonic systems at low temperatures are also known to posses features that can be interpreted as being of a topological order nature. For instance, electric charge polarization of the vacuum, in a manner similar to those found on systems such as topological insulators, has been reported for 2-dimensional QED systems [6-8]. At this point it is important to clarify that, even though the concept of topological order cannot be considered to be a consensus among the physics community, 
the properties we have already mentioned above are the ones we will understand by topological order throughout this document.

As expressed before, it is our understanding that topological order is still not fully characterized and remains a working definition. However, broadly speaking (and by no means trying to be complete), it is possible to recognize the following subtypes:

- Long Range Entanglement (LRE): In this case, there are entangled states that cannot be separated without changing the topology in which the system is defined;

- Symmetry Protected Topological order (SPT): In this case, there are entangled states that cannot be separated without breaking the symmetry that allows them to exist.

This work is mostly concerned with the first case (LRE) as the models we present have this characteristic.

\subsection{Characterization and classification}

The discovery of the aforementioned systems with topological order has encouraged the theoretical study and their subsequent description since the early 1980's. A common theoretical approach has been to build broad classes of exactly soluble models with topological features. Our results are in line with this research, representing a systematization of a general subclass of models with abelian properties. However, the relevance of these models must be understood as not necessarily describing physical systems accurately. Instead, in the spirit of having at hand many computable toy models that are inspired by physical systems, all of them have key topological features observed experimentally. The situation resembles the way in which models like the Ising model or the Potts model have been important for their study in statistical mechanics.

In spite of the successes achieved by means of modeling described above, two problems remain to be solved: the complete characterization and the subsequent classification. These are of uttermost importance for both the condensed matter community and the quantum information community. While the condensed matter interest is obvious, the quantum information community's interest comes from the fact that many of the toy models studied can be interpreted as quantum codes with robust characteristics and promising implementation possibilities. As for the first problem, topological phases are partially described by Topological Quantum Field Theories (TQFTs) [9, 10]. Thus, in principle, an identification of TQFTs that can possibly describe gapped phases of gauge 
theories can be used as a means of a partial classification of topological phases. In the absence of any further global symmetry and for the case of gapped phases of finite 1gauge theories, a famous classification of TQFTs has been given by Dijkgraaf and Witten $[9,11]$. Concretely, Dijkgraaf-Witten topological actions with gauge group $G$ have been used to classify gapped phases of matter with global symmetry group $G$. In fact, this is the current understanding of what is known as symmetry protected topological (SPT) phases [12-17] which is described by the group cohomology classification of SPT phases [18]. A more general classification of SPT phases was proposed in [19]. Furthermore, it was argued that the ground state could depend on a (emergent) discrete symmetry that was never identified explicitly, except of being $\mathbb{Z}_{n}$ for the FQH. These questions are not going to be tackled over the course of this research as we prefer to focus on the formulation and understanding of abelian topological systems. Also, it is worth to mention the works of [20], where bosonic lattice realizations of SPT phases with higher form symmetry are presented, and [21, 22], where is discussed their connection to fault-tolerant logical gates in topological quantum codes.

A particular theoretical interest has been given to the two dimensional models, which have been studied thoroughly. Some models have been particularly popular, notably the string nets [23] and the quantum double models (QDM) [24-26]. The latter is particularly important for the formalism that will be presented in this work. In a nutshell, quantum double models can be understood as a many-body system in a two dimensional lattice. The most simple and well-known of these models is the so called Toric Code (TC) [24, 27], which can be interpreted either as a many body interacting system over a 2 -dimensional lattice or as a lattice gauge theory with $\mathbb{Z}_{2}$ gauge group. The Toric Code emerged initially as a correction code in the context of quantum computation and was later realized its potential as a suitable toy model illustrating topological order. Furthermore, the ground state is degenerate and directly dependent on the topology of the lattice. When the embedding surface of the lattice is a Torus, the ground state is known to have a 4 -fold degeneracy. Completing the picture, when local perturbations are applied to the ground state, it exhibits excitations that can be interpreted as quasiparticles with exotic statistics (anyons) that regain bosonic and/or fermionic statistics when braided among themselves.

The TC situation stated in the previous paragraph is generally true for more QDM: from a condensed matter perspective, they can be interpreted as a spin quantum system and, from a field theoretic perspective, as a $(2+1) d$ gauge theory. Their exotic statistics (mostly localized quasi particles) are determined by representations of the braid group, elements of which are called anyons. However, the generalization from the two dimensional models to the three dimensional case is far from being straightforward and has met some non-trivial problems on the way. For instance, anyon excitations, which 
appear naturally in the two dimensional case, are not smoothly translated into three or four dimensional cases, due mainly to the non-equivalence of their respective topologies. We can argue that since particles (point like objects) in three dimensions can only behave as bosons or fermions, should anyonic behavior exist, it must come from extended objects (one dimensional or two dimensional objects) [28]. Hence, in higher dimensions, in order to have exotic statistics, the quasi particles may be represented by loops, knots, surfaces, etc. This presents a threat to the elegant classification given in the two dimensional case by the braid group and foreshadows the appearance of other different structures like the motion group and the loop braid group [29-31] in higher dimensions. For this reason, the possibility of finding analogs of the two dimensional anyons is considerably reduced, and several works are intended to study and characterize the representations of the aforementioned groups, see for instance [32, 33].

However, not everything has been half successful on the field. One of the most important triumphs of the last decades in mathematical physics has been produced when taking a field theory perspective. In fact, when viewed as gauge theories on the lattice, these models have allowed to define new quantities that are shown to be topological invariants [34-37]. This has fostered interactions between the related physics community and the algebraic topology community. In fact, the results presented here are manifestly algebrotopological in their essence.

\subsection{Our motivation}

We have mentioned some of the phenomenological motivations to study these systems, but we want to emphasize that our interest remains mathematico-theoretical and can be summarized as follows:

(i) The models presented have proven to be a suitable machinery to build toy models with desired topological order characteristics and, to be of great importance to condense matter physics [5, 38]. In addition, they are greatly appreciated in theoretical and mathematical physics through their applications in the field of quantum computation $[24,27]$.

(ii) On a more technical level, the mathematical tools employed are generally varied and far-reaching. For instance, certain approaches have been developed using fusion categories, representation categories, Hopf algebras and homology/cohomology theory techniques, among others. This feature gives the modeling task a big possibility of exploration [39-42]. 
Consequently, we take the route of algebraic topology to construct and study a class of models that live in arbitrary dimensions and therefore, escape the Dijkgraaf and Witten classification. We will see that, by sacrificing the non-commutativity of the degrees of freedom, we are able to produce models involving generalized gauge fields in all dimensions. Furthermore, the most important feature of this formalism is that it clarifies, by means of an explicit expression, the connection between the topology and the ground state subspace of the model. Moreover, related algebro-topological techniques allow us to characterize the ground state subspace in a comprehensive way. The models describe gapped phases of abelian gauge theories and can be interpreted as describing topological phases of matter [43-45] when taking the geometrical chain complex to be a discretization of a compact manifold. Thus, our construction allows us to get a variety of models exhibiting intrinsic topological order $[23,46,47]$ that can be thought of as prototypical models of topological phases of matter in higher dimensions. Great interest regarding such models arose due to possible applications in fault tolerant quantum computation [24, 48, 49].

\subsection{Outline}

We emphasize that, in an attempt to present a mostly self-contained document, we have included several Appendices with technical information to facilitate the reading experience of this thesis. As for the body of this work, the document is organized as follows:

(1) In order to get a grasp of the geometrical ideas behind the construction presented here, we dedicate Chapter 2 to review the most important features of the traditional approach to gauge theories. The notion of a gauge group will be later replaced by a more general mathematical object, namely, a chain complex of abelian groups. By the end of the Chapter, we include generalizations of these ideas that involve categorical methods. We will see later that these theories, in their abelian version, are described by our formalism.

(2) In Chapter 3, we discuss the types of models from which we have drawn most of the inspiration for proper generalization, the quantum double models defined in two dimensions. As a motivational section, by the end of the chapter, we also include a glimpse of some successful attempts, developed by our research group, to consider degrees of freedom beyond the traditional gauge, based on Hopf algebraic methods. The latter represent much of the focus of the research group we are part of. Those were discussed in several publications $[50,51]$ and ignited many of the ideas that turned out to be relevant in the present discussion. 
(3) In Chapter 4, we introduce the formalism developed. Here, the notion of gauge configuration is replaced by the notion of maps between two chain complexes. It will be seen that, in this context, it is quite natural to construct a Hamiltonian formulation of generalized gauge theories that can be defined in arbitrary dimensions.

(4) In Chapter 5, we study the ground state of these models. We show them to have a degenerate ground state subspace whose basis elements are in one-to-one correspondence with the cohomology classes having coefficients in the chain complex of abelian groups [52]. Furthermore, this formalism allows to explicitly show that the ground state degeneracy (GSD) is a topological invariant, for which we give a closed formula in terms of the 0-th cohomology group with coefficients in the chain complex of abelian groups. Moreover, due to a theorem by Brown [52], the GSD can be understood as exhibiting contributions from each dimension.

(5) Finally, in Chapter 6, we discuss possible branchings of this work as well as prospective applications of the same.

To finalize this introduction, it is important to say that most of the material presented is currently being peer reviewed as a paper representing the outcome of a collaborative effort between Pablo Ibieta Jimenez, Ricardo Almeida and Paulo Teotônio-Sobrinho, the first two of whom are colleagues in the research group led by the latter and all of whom I am greatly indebted and thankful to.

\section{J. Lorca Espiro.}




\section{Chapter 2}

\section{Gauge Theory Fundamentals}

This chapter aims to be introductory and motivational. We will briefly discuss some general notions of gauge theories, mainly from a physical point of view without entering in technical subtleties. Following Wilson [53] in his seminal paper, we will describe the fundamentals of a discretization of Quantum Field Theories (QFT), leading to what is known as Lattice Gauge Theories ( $L G T$ ) with the aim of extrating the most fundamental ideas to later generalize in the following chapters.

We will be focusing in the continuous theory. By this we mean the Field Theory that assumes the continuum of the space-time manifold or, differently phrased, the fabric in which the physical events will occur are considered to be continuous. From this perspective, a Lagrangian Functional $\mathcal{L}$ with the desired physical information and/or restrictions can be constructed and hence the dynamics of the model are easily obtained through the field equations derived from it.

A word of warning is needed at this moment: The gauge theories, as presented here, are endorsing an approach which, in a sense, is the "inverse problem" of the procedure we will be presented in the later chapters. The difference lies in that, since in this chapter the Lagrangian/Hamiltonian functional is assumed to exist from first principles, it contains all information to derive the dynamical equations. On the contrary, in our formalism, the Lagrangian/Hamiltonian is ad hoc, to fulfill certain mathematical requirements. Thus, the Lagrangian/Hamiltonian is obtained through direct calculation involving the basic operators of the theory, as it will be shown in later chapters. 


\subsection{Brief review of the differentio-geometric approach to gauge theories}

Remark 2.1.1. Before starting this first section we recommend to go through the summary of topology and differential geometry of Appendix A). It is hoped that this will suffice to understand this (very brief and non-technical) description of the mathematical structure of a gauge theory in its continuous version and later on its discrete analog.

The geometrical support assumed in these theories is recognized as a bundle, which we describe next:

Definition 2.1.2 (bundle). A bundle can be considered as a natural extension of the concept of manifold and consist of the triple $(E, p, M)$ where $E$ is called the total space, $M$ is the base space of the bundle, usually a smooth manifold, and the map $p: E \rightarrow M$ which is called the projection.

Furthermore, for each $x \in M$, the inverse map $p^{-1}(x)$ is the called the fiber (or fibre) of the bundle at the point $x$. In this way we can define a cross section to be a map $s: M \rightarrow E$ such that $(p \circ s)(x)=x$ for each $x \in M$, that is, locally $s(x)=p^{-1}(x)$. Let us denote the fiber by $E_{x}:=p^{-1}(x)$. It is clear that each fiber can be provided with some further mathematical structure, i.e. being a vector space, a tensor space, a group, etc. Hence, when the operations defined on the fiber $E_{x}$ vary smoothly from fiber to fiber, $p$ is a called vector, tensor, etc - bundle respectively.

Following the canonical construction, we need provide a way to connect and operate between different bundles, this is done through what is known as bundle maps:

Definition 2.1.3 (Bundle maps). A bundle map is a pair of maps $\alpha$ and $\bar{\alpha}$ that makes the diagram of figure 2.1 commuting

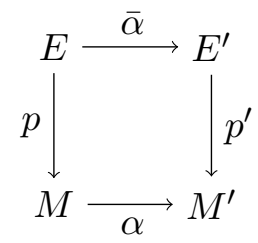

Figure 2.1: Commuting bundle map

In other words, for maps between bundles, the previous commutative diagram implies that we require that for each $x \in M, \bar{\alpha}: E_{x} \rightarrow E_{\alpha(x)}$ behaves as a morphism in the 
respective structure (linear map, in the case of vector bundles, group homomorphism in the case of bundles of groups, etc.).

Notice that, because of the bundle encompassing the manifold $M$ as well, its mathematical structure is translated into the bundle. Therefore, when the manifold is smooth, it allows us to define the exterior derivative and mainly the entire apparatus of differential geometry. On the other hand, apart from the exterior derivative, defined over a manifold, on a bundle we can define the covariant derivative:

Definition 2.1.4 (Covariant derivative). Let $E$ be a smooth vector bundle over a differentiable manifold $M$. Let us also denote the space of smooth sections of $E$ as $s(E)$. A connection on $E$ is an $\mathbb{R}$-linear map $\nabla: s(E) \rightarrow s\left(E \otimes T^{*} M\right)$ such that the Leibniz rule $\nabla(\sigma f)=(\nabla \sigma) f+\sigma \otimes d f$ holds for all smooth functions $f$ on $M$ and all smooth sections $\sigma$ of $E$.

This is a well known differential operator in different areas of physics, specially in General relativity, where the connection is related to the Christoffel symbols [54] of the local expressions, which are generally known in the coordinate free language as affine connections. Moreover, since this presentation is coordinate independent, it allows to define a covariant derivative beyond the affine ones. In fact, this is evident when considering $\mathbf{X}$, a tangent vector field on $M$ (i.e. a section of the tangent bundle $T M$ ). One can then define a covariant derivative along $\mathbf{X}$, i.e. $\nabla_{\mathbf{X}}: s(E) \rightarrow s(E)$, by contracting $\mathbf{X}$ with the resulting covariant index in the connection $\nabla$ (i.e. $\nabla_{\mathbf{X}} \sigma=(\nabla \sigma)(\mathbf{X})$ ). Thus defined, the covariant derivative satisfies the following properties:

(i) Linearity on the argument

$$
\nabla_{\mathbf{X}}\left(\sigma_{1}+\sigma_{2}\right)=\nabla_{\mathbf{X}} \sigma_{1}+\nabla_{\mathbf{X}} \sigma_{2}, \text { for } \sigma_{1}, \sigma_{2} \in s(E)
$$

(ii) Linearity on the connection space

$$
\nabla_{\mathbf{X}_{1}+\mathbf{X}_{2}} \sigma=\nabla_{\mathbf{X}_{1}} \sigma+\nabla_{\mathbf{X}_{2}} \sigma, \text { for } \mathbf{X}_{1}, \mathbf{X}_{2} \in T M,
$$

(iii) Leibniz rule

$$
\nabla_{\mathbf{X}}(f \sigma)=f \nabla_{\mathbf{X}} \sigma+\mathbf{X}(f) \sigma, \text { for } f \text { a zero form and }
$$

(iv) Natural for zero-forms $\nabla_{f} \mathbf{X} \sigma=f \nabla_{X} \sigma$

Conversely, any operator satisfying the above properties defines a connection on $E$, and a connection in this sense is also known as a covariant derivative on $E$. This gives rise to the concept of gauge transformation: 


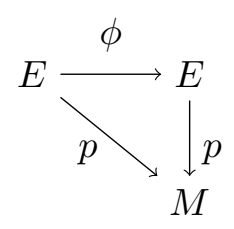

Figure 2.2: A gauge transformation

Definition 2.1.5 (Gauge transformation). A gauge transformation of a bundle $\phi: E \rightarrow$ $M$ is a just a bundle automorphism (with the identity map on the base space) as is shown in the diagram of figure 2.2

Among all the possible structures of bundles, the $G$-bundle is the one considered when constructing a gauge theory with applications in physics. We pass on to define it:

Definition 2.1.6 ( $G$-bundle). Consider the bundle $(E, p, Y)$ and let $G$ be a Lie group. We identify the manifold $Y$ with a right (resp. left) $G$-space, with a smooth right $G$-action $\mu$ such that for $g \in G$ and $y \in Y$

$$
\begin{aligned}
& \mu: E \rightarrow E, \\
& \mu:(y, g) \mapsto y \triangleleft g,
\end{aligned}
$$

where $\triangleleft$ as the right action of $g \in G$ over $y \in Y$.

We call the smooth map $f$ a $G$-equivariant map if $f: E \rightarrow E$ is such that $f(y \triangleleft g)=$ $f(y) \triangleleft g \forall y \in E$ and $g \in G$. A $G$-bundle is said to be locally trivial if $E$ is a $G$-space in which the action of $G$ preserves fibers, i.e. $y \in E_{x}$ implies $y \triangleleft g \in E_{x}$ for every $g$. This makes each fiber into a $G$-space. If $E$ is a $G$-bundle, the action of $G$ gives, for each element $g \in G$, a gauge transformation showed in figure 2.3, which represents a

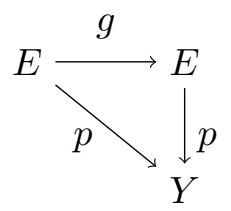

Figure 2.3: Diagram of a global Gauge transformation for a $G$-bundle

global gauge transformation. It is worth to mention that there are more general gauge transformations than these, one for every invertible $G$-equivariant map from $E$ to $E$.

We stop in a particular class of $G$-bundles, in fact, the most important kind. These are the basic structure of most of the physics descriptions of gauge theories such as the Yang-Mills Theory: 
Definition 2.1.7 (Principal $G$-bundle). A principal $G$-bundle is a $G$-bundle satisfying that for any $y, y^{\prime} \in E_{x}$ there exits a unique $g \in G$ such that $y \triangleleft g=y^{\prime}$. Note also that if we have $y_{o} \in E_{x}$, the map

$$
\begin{aligned}
& \mu: E_{x} \times G \rightarrow E, \\
& \mu:\left(y_{o}, g\right) \mapsto y_{o} \triangleleft g,
\end{aligned}
$$

is an isomorphism of $G$-spaces. In other words, a principal $G$-bundle is a locally trivial bundle of $G$-spaces, where every fiber is isomorphic, as a right- $G$-space, to $G$.

\subsection{Traditional approach to gauge theory}

We will readily present the formalism on which traditionally gauge theories have been constructed. The treatment here presented closely follows the one on [39]. We can sum up the constituents of a Gauge Theory as possesing mainly three basic ingredients:

(i) $M$ : an $n$-manifold, i.e. Space-Time;

(ii) $G$ : a compact Lie group, i.e. a gauge group;

(iii) $P$ : a principal $G$-bundle,

where a local trivialization $P \simeq M \times G$ of the principal $G$-bundle is used. This trivialization is known as a gauge in the physics context. Let us denote the set of $p$-forms defined on this trivial fiber bundle $P$ to be $\Omega^{p}(P)$. Henceforth, the basic dynamical variable is a connection 1-form $\mathbf{A} \in \Omega^{1}(P)$ called the gauge field that takes account of the parallel transport over the bundle.

In this trivialization, the curvature $\mathbf{F} \in \Omega^{2}(P)$ is defined to be

$$
\mathbf{F}:=d_{\mathbf{A}} \mathbf{A}=d \mathbf{A}+[\mathbf{A} \wedge \mathbf{A}],
$$

where $[\cdot \wedge \cdot]$ is a Lie bracket that takes values over the Lie algebra elements. We also introduce the notation for the covariant derivative to be $d_{\mathbf{A}}(\cdot)$, in this case, applied to the connection 1-form A. Now, if a map $h: M \rightarrow G$ is considered to be acting on the dynamical variables, it can be shown that they transform locally in the following way

$$
\begin{aligned}
\mathbf{A} \mapsto \mathbf{A}^{\prime} & =h^{-1} \mathbf{A} h+h^{-1} d h, \\
\mathbf{F} \mapsto \mathbf{F}^{\prime} & =h^{-1} \mathbf{F} h
\end{aligned}
$$


This operation is called a Gauge Transformation.

Over the previous set up an Action Functional

$$
\mathcal{S}=\int_{M} \mathcal{L}
$$

can be constructed through the use of a Lagrangian Density $\mathcal{L}=\mathcal{L}(\mathbf{A}, \mathbf{F}) \in \Omega^{4}(P)$. This functional encodes the entire theory, the information contained is made explicit by deriving the equations of motion through the variational principle $\delta S=0$. Moreover, the symmetries of the theory are imposed directly over the Lagrangian. The gauge symmetry can be characterized by the relation

$$
\delta \int_{M} \mathcal{L}(\mathbf{A}, \mathbf{F})=\delta \int_{h^{*} M} \mathcal{L}\left(\mathbf{A}^{\prime}, \mathbf{F}^{\prime}\right)=0,
$$

where the, already introduced, map $h$ was used, as well as the transformation equations (2.4). Therefore, the term gauge refers to the redundant degrees of freedom when the equations of motion are insensitive to the gauge transformation. The gauge freedom is thus, incorporated as a representation of a Lie group, directly into the Lagrangian density, referred to as the symmetry group or the gauge group of the theory. Every Lie group has a Lie algebra of group generators associated and for each group generator necessarily arises a corresponding vector field called the gauge field.

Let us now consider a parallel transport over a path $\gamma:[0,1] \rightarrow M$, such that the following differential equation holds for a cross section $s: M \rightarrow P$ of the bundle

$$
d_{\mathbf{A}} s=0,
$$

geometrically speaking this means that the section $s$ stays in the same direction as the tangent vector $\frac{d \gamma}{d t}$ along its path, as well as not changing the size. Using the same local trivialization exposed before, the solution to equation (2.7) is formally expressed through the path ordered integral

$$
\operatorname{hol}(\gamma):=g_{\gamma}=\mathcal{P} \exp \left(-\int_{\gamma} \mathbf{A}\right)
$$

where $\mathcal{P}(\cdot)$ stands for a path ordering operator, where $\gamma(0)=e$ the identity element, and $\gamma(1)=g_{\gamma}$. It can be shown that, when the map $h$ is acting throughout the path, this quantity is modified according to the following conjugation rule

$$
g_{\gamma}^{\prime}=h^{-1}(\gamma(0)) g_{\gamma} h(\gamma(1))
$$


Note that if the path $\gamma=\bar{\gamma}$ is a closed loop, i.e. $\bar{\gamma}(0)=\bar{\gamma}(1)$, therefore

$$
g_{\bar{\gamma}}^{\prime}=h^{-1} g_{\bar{\gamma}} h
$$

which turns this quantity into a class function.

The class function hol $(\gamma)$ defined in equation (2.10) is called holonomy around the path $\gamma$ and is intimately related to the curvature previously defined. We will briefly show this by introducing an order parameter $\epsilon \in \mathbb{R}$ such that the re-scaling $\mathbf{A} \rightarrow \epsilon \mathbf{A}$ takes place. Let us also consider a closed loop $\bar{\gamma}=\partial \sigma$ where $\sigma$ is a 2-cochain. In this case, the following quantities have an explicit dependency on $\epsilon$ as

$$
\begin{aligned}
g_{\partial \sigma}(\epsilon) & =\mathcal{P} \exp \left(-\epsilon \int_{\partial \sigma} \mathbf{A}\right)=\mathbf{I}-\epsilon \int_{\partial \sigma} \mathbf{A}+\epsilon^{2} \int_{\partial \sigma^{1}} \int_{\partial \sigma^{2}} \mathcal{P}\left(\mathbf{A}_{1} \mathbf{A}_{2}\right)-\mathcal{O}\left(\epsilon^{3}\right), \\
F_{\sigma}(\epsilon) & =\int_{\sigma} \mathbf{F}(\epsilon, \mathbf{A})=\epsilon \int_{\partial \sigma} \mathbf{A}+\epsilon^{2} \int_{\sigma}[\mathbf{A} \wedge \mathbf{A}],
\end{aligned}
$$

so that to first order the following relation holds

$$
\left.\frac{d}{d \epsilon} g_{\partial \epsilon}(\epsilon)\right|_{\epsilon=0}=-\left.\frac{d}{d \epsilon} F_{\sigma}(\epsilon)\right|_{\epsilon=0}
$$

where we have used Stokes' theorem for the first order terms in order to make the equality explicit. Equation (2.11) shows that, up to first order, holonomy $g_{\partial \sigma}$ and the integration of the curvature $\mathbf{A}$ over the 2-cochain $\sigma$ have essentially the same information. This fact will be utterly important on the construction of a lattice gauge theory, which is basically a first order theory. In a sense, holonomy can be considered to have the basic information of the gauge theory in terms of the parallel transport and, moreover, it is a class function from which observables of the theory can be built from.

A condition known as flat connection

$$
d_{\mathbf{A}}=\mathbf{F}=0
$$

is used very often. We can interpret this condition in the following way: Imagine a parallel transport of a section $s$ of the bundle from the same point $p_{0}$ to the point $p_{11}$, following the direction $\frac{d \gamma_{1}}{d t}$ where $\gamma_{1}$ is a path over the manifold $M$. Now, from the same point $p_{0}$ consider a second direction $\frac{d \gamma_{2}}{d t}$ and perform a parallel transport to the point $p_{21}$, where again $\gamma_{2}$ is a path over the manifold $M$. Now, from the point $p_{11}$ perform a parallel transport following the direction $\frac{d \gamma_{2}}{d t}$ to the point $p_{12}$, and same thing from the point $p_{21}$ to the point $p_{22}$ following the direction $\frac{d \gamma_{1}}{d t}$. The previous situation is depicted in figure 2.4 . 

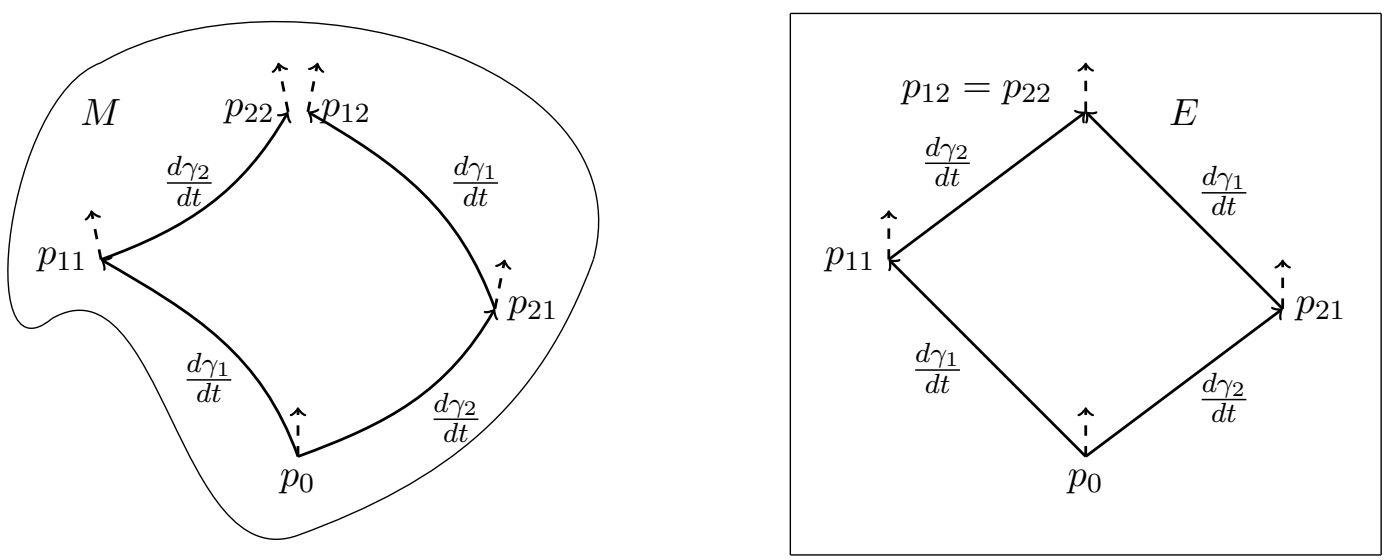

FigURE 2.4: "Parallelepiped" over a bundle $P$ with negative curvature vs a "paralleliped" in a Euclidean space $E$ (null curvature), the dashed arrow represents the fiber in each case

Remark 2.2.1. In a flat (Euclidean) space this operation is equivalent to construct a parallelepiped in which the points $p_{12}$ and $p_{22}$ will coincide, however in a general geometry, like for instance a bundle, this is not the case and the difference between these two points up to second order (i.e. $\mathcal{O}\left(\epsilon^{2}\right)$ ) is the curvature $\mathbf{F}$. Hence, by imposing flat connections we are considering a difference in this construction up to a first order (i.e. $\mathcal{O}(\epsilon)$ ), which is consistent with identifying the holonomy as having the information of the curvature.

Let us remember that holonomy is a class function, thus, a function $\sigma$ of the type $\sigma\left(g_{\gamma}\right)=\sigma\left(h^{-1} g_{\gamma} h\right)$ where the holonomy is calculated over a loop, is also a class function. One remarkable feature of class functions is that according to group theory, any such function can be expanded in characters of irreducible representations. Recalling that an irreducible representation (or irrep) is a nonzero representation that has no proper subrepresentation. Consequently, we can expand the holonomy as

$$
\chi_{V}\left(g_{\gamma}\right) \doteq \sum_{i}\left\langle\nu_{i}^{*}\left|\rho_{V}\left(g_{\gamma}\right)\right| \nu_{i}\right\rangle
$$

where $\nu_{i}$ is the basis of the representation space $V$ and $\nu_{i}^{*}$ its dual basis, $\rho_{V}\left(g_{\gamma}\right)$ denotes the matrix representation matrix. Thus, the assignment $\mathbf{A} \mapsto \chi_{V}\left(g_{\gamma}\right)$ is called a Wilson loop. 


\subsection{The categorical view of gauge theories}

Although the material covered so far is quite standard, we embark ourselves on a presentation of a less well known approach with focus on generalizing its main features. We will refer this program as the $n$-group approach to higher dimensional gauge theories. We will see that it uses a different level of abstraction by the means of introducing some category theory, more specifically the idea of a functor. It represents a more efficient way to describe the features the construction discussed up to now. However, its generalization to higher dimensions is not exempt of technical problems, for which we will deal at the end of the Chapter.

\subsubsection{The 1-gauge}

In this case, we can understand a connection for any parallel transport along a path as being a functor that sends a path groupoid to the same object of a group and every morphism in the path groupoid to an element of the group. The previous idea can be formalized in the following theorem $[55,56]$

Theorem 2.3.1 (hol Functor). For any Lie group $G$ and any smooth manifold $M$, there is a one-to-one correspondence between:

(1) Connections on the trivial principal G-bundle over $M$.

(2) $\mathfrak{g}$-valued 1-forms on $M$, where $\mathfrak{g}$ is the Lie algebra of $G$, and

(3) Smooth functors hol: $\mathcal{P}_{1}(M) \rightarrow G$, where $\mathcal{P}_{1}(M)$ is the path groupoid of $M$.

Since we have covered the first two points in the previous section, we will concentrate on the third one. We start by discussing the concept of path groupoid. Suppose $M$ is a manifold. Then the path groupoid $\mathcal{P}_{1}(M)$ is a roughly a category in which objects are points of $M$ and a morphism from $x$ to $y$ is a path from $x$ to $y$. We compose paths by gluing them end to end. Hence, given a path $\delta$ from $x$ to $y$, and a path $\gamma$ from $y$ to $z$ :

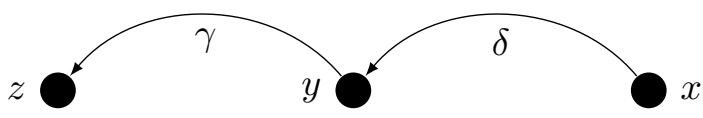

Figure 2.5: Composition of paths $\gamma: y \rightarrow z$ and $\delta: x \rightarrow y$

such that, the composition $\gamma \delta$ is a path from $x$ to $z$, built from $\delta$ and $\gamma$.

However, a technical detail arises when considering this composition to be well defined and associative. We then consider either piecewise smooth paths or lazy paths, which 


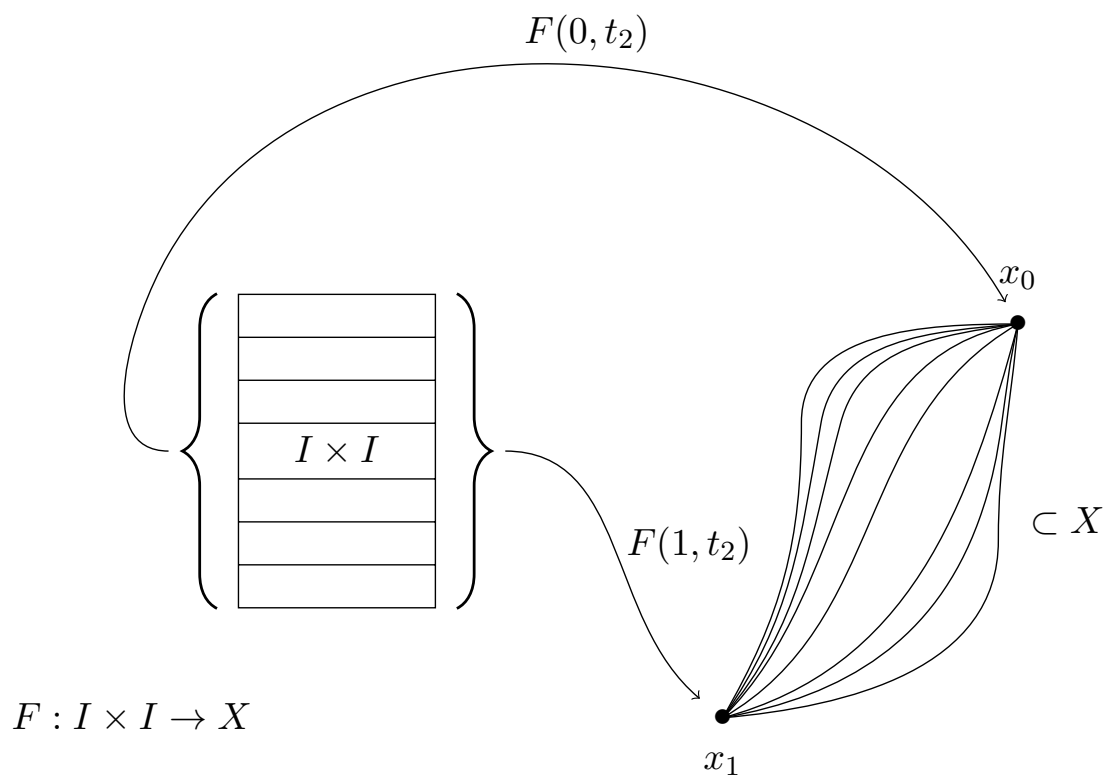

Figure 2.6: Homotopic paths in $X$ space between $x_{0}$ and $x_{1}$.

we will describe in what follows. A path $\gamma:[0,1] \rightarrow M$ is said to be lazy if it is smooth and also constant in a neighborhood of $t=0$ and $t=1$. In this way, composition can be defined in the usual way

$$
\gamma \delta:[0,1] \rightarrow M \quad(\gamma \delta)(t)=\left\{\begin{array}{l}
\delta(2 t) \quad \text { if } 0 \neq t \neq \frac{1}{2} \\
\gamma(2 t-1) \quad \text { if } \frac{1}{2} \neq t \neq 1
\end{array}\right.
$$

therefore, by taking $\gamma$ and $\delta$ to be lazy its composition is also lazy, i.e. closed under composition. However, in order for the composition operation to be associative we need to take homotopy equivalence classes of lazy maps as follows. We say that two lazy paths $\delta$ and $\gamma$ are homotopic, or lie in the same homotopy class if there is a smooth map

$$
\Sigma:[0,1]^{2} \rightarrow M \quad \text { such that } \quad \begin{aligned}
\Sigma(0, t) & =\gamma(t), \\
\Sigma(1, t) & =\delta(t)
\end{aligned}
$$

with its associated diagram

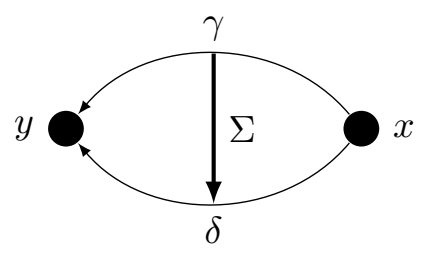

Figure 2.7: Homotopic paths $\delta: y \rightarrow x$ and $\gamma: y \rightarrow x$

However, if we are to associate connections to these paths, parallel transport along 
homotopic classes of lazy paths gives different results unless the connection is flat, i.e. has zero curvature. This forces for a more ellaborate equivalence between paths. Hence, the concept of thin homotopy arises. A thin homotopy is one that sweeps out a surface that has zero area. In other words, if $\Sigma$ such that the rank of the differential $d \Sigma$ is less than 2 at every point. If two paths differ by a smooth reparametrization, they are thinly homotopic. Given the latter considerations, we define the path groupoid $\mathcal{P}_{1}(M)$ as:

Definition 2.3.2 (Path groupoid). The path groupoid $\mathcal{P}_{1}(M)$ is the category where:

(1) Objects are points in $M$

(2) Morphisms are thin homotopy classes of lazy paths in $M$

(3) Denoting the thin homotopy class of $\gamma$ as $[\gamma]$, composition is defined by $[\gamma][\delta]=$ $[\gamma \delta]$, for two thin homotopy classes $[\gamma]$ and $[\delta]$ in $M$.

(4) For any point $x \in M$, the identity $1_{x}$ is the thin homotopy class of the constant path at $x$.

To be precise, we can regard a group to be a groupoid with only one object, that is, a category where every morphism $\gamma: x \rightarrow y$ has an inverse $\gamma^{-1}: y \rightarrow x$ satisfying $\gamma \gamma^{-1}=1_{x}$ and $\gamma^{-1} \gamma=1_{y}$. In $\mathcal{P}_{1}(M)$, the inverse is defined using the concept of a reverse path as $[\gamma]^{-1}:=\left[\gamma^{-1}\right]$. In fact, let us take a group $G$, then it can be regarded as a category in the following way:

(i) There is only one object

(ii) Morphisms from $\bullet$ to $\bullet$ are elements of $G$

(iii) Composition of morphisms is multiplication in the group $G$

(iv) The identity morphism 1 • is the identity element of $G$

The groupoid status is ensured by the fact every element has an inverse. Conversely, any groupoid with one object is a group, so these two notions will be used interchangeably.

Continuing with the construction, we recall that a connection gives a map from $\mathcal{P}_{1}(M)$ to $G$, which says how to transform a particle when we move it along a path. More precisely: if $G$ is a Lie group, any connection on the trivial $G$-bundle over $M$ yields a map We recognize that to be the holonomy 2.8.

The functor hol must send all the points of $M$ to a unique object of $G$. More interestingly, it must send thin homotopy classes of paths in $M$ to elements of $G$, such that preserves 


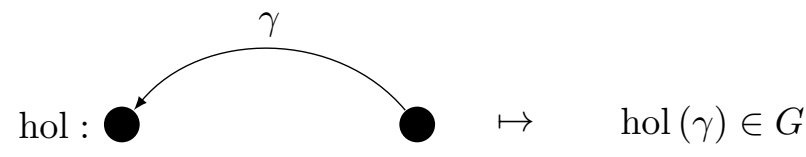

composition, i.e. :

$$
\operatorname{hol}(\gamma \delta)=\operatorname{hol}(\gamma) \operatorname{hol}(\delta)
$$

for any two thin homotopy paths $\gamma, \delta$ in $M$, and identities:

$$
\operatorname{hol}\left(1_{x}\right)=1 \in G \text {. }
$$

The former properties can be seen to be fulfilled by connections. Indeed, when computing the variation of hol $(\gamma)$ when the path $\gamma$ is varied, it can be shown that is null if the homotopy is thin. The composition preservation is obvious from the composition of paths and finally, the path-ordered exponential along a constant path is just the identity, as expected.

The only additional condition that needs to be imposed is that hol $(\gamma)$ depends smoothly on the path $\gamma$, but if this condition is dropped, a generalized connection on a smooth manifold $M$ can be actually defined to be the functor hol : $\mathcal{P}_{1}(M) \rightarrow G$. Furthermore, this construction has been used in loop quantum gravity to define a natural measure on the space of generalized connections, so a Hilbert space of complex-valued squareintegrable functions on the space of generalized connections [57-59]. However, in the continuous case, it is possible to use the theory of smooth groupoids, since any Lie group is a smooth groupoid, and so is the path groupoid of any smooth manifold.

Heuristically, suppose we have a smooth function $F:[0,1]^{n} \times[0,1] \rightarrow M$, which can be thought of as a parametrized family of paths. And suppose that for each fixed value of the parameter $s \in[0,1]^{n}$, the path $\gamma_{s}$ given by $\gamma_{s}(t)=F(s, t)$ is lazy. Then our functor hol : $\mathcal{P}_{1}(M) \rightarrow G$ gives a function

$$
\begin{aligned}
{[0,1]^{n} } & \rightarrow G \\
s & \mapsto \operatorname{hol}\left(\gamma_{s}\right) .
\end{aligned}
$$

If this function is smooth whenever $F$ has the above properties, then the functor hol : $\mathcal{P}_{1}(M) \rightarrow G$ is smooth. Hence, starting from this comment, the following lemma can be proven:

Lemma 2.3.3. There is a one-to-one correspondence between smooth functors hol: $\mathcal{P}_{1}(M) \rightarrow G$ and Lie $(G)$-valued 1 -forms $A$ on $M$. 
which is the basis of the Theorem 2.3.1 that allows the entire construction we have been discussing up to now.

\subsubsection{The 2-gauge}

As a motivation, we also review the construction of the so called 2-gauge connections. The main idea is that a connection is a way to describe how particles transform as they move along paths. A 2 connection will tell how 2-dimensional objects, namely strings, transform when they sweep out surfaces. The generalization from the construction of the 1-gauge case to the 2-gauge case is enclosed in the following Theorem:

Theorem 2.3.4 (hol 2-Functor). For a Lie 2-group $\mathcal{G}$ and any smooth manifold $M$, there is a one-to-one correspondence between:

(i) 2-connections on the trivial principal $\mathcal{G}$-2-bundle over $M$,

(ii) pairs consisting of a smooth $\mathfrak{g}$-valued 1 -form $A$ and a smooth $\mathfrak{h}$ valued 2 -form $B$ on $M$, such that

$$
\underline{t}(B)=d A+A \wedge A
$$

where we use $\underline{t}: \mathfrak{h} \rightarrow \mathfrak{g}$, the differential of the map $t: H \rightarrow G$, to convert $B$ into a $\mathfrak{g}$-valued 2 -form, and

(iii) smooth 2-functors

$$
h o l: \mathcal{P}_{2}(M) \rightarrow \mathcal{G}
$$

where $\mathcal{P}_{2}(M)$ is the path 2-groupoid of $M$.

Although we will not prove this theorem, we will try to dissect all its components in order to have a grasp of the physical model behind it. We proceed heuristically, as we did in the previous section. We begin by describing the 2-path groupoid:

Definition 2.3.5 (2-groupoid). A 2-groupoid is a 2 category where:

(a) Every morphism $f: x \rightarrow y$ has an inverse, $f^{-1}: y \rightarrow x$, such that

$$
f^{-1} f=1_{x} \quad \text { and } \quad f f^{-1}=1_{y}
$$

(b) Every 2-morphism $\alpha: f \Rightarrow g$ has a vertical inverse, $\alpha_{\text {vert }}^{-1}: f \Rightarrow g$, such that

$$
\alpha_{\text {vert }}^{-1} \cdot \alpha=1_{f} \quad \text { and } \quad \alpha \cdot \alpha_{\text {vert }}^{-1}=1_{f}
$$


(c) Every 2-morphism $\alpha: f \Rightarrow g$ has vertical inverse, $\alpha_{\text {hor }}^{-1}: f^{-1} \Rightarrow g^{-1}$, such that

$$
\alpha_{\text {hor }}^{-1} \circ \alpha=1_{1_{x}} \quad \text { and } \quad \alpha \circ \alpha_{\text {hor }}^{-1} \circ \alpha=1_{1_{x}} .
$$

Consequently, and in an analogous way as described for a path (1) groupoid, a path 2-groupoid $\mathcal{P}_{2}(M)$ of a manifold $M$ are thin homotopy classes of lazy surfaces, as a somewhat naive generalization. Just as a group (1-group) was a groupoid with one object, we define a 2-group to be a 2-groupoid with one object. The structure of a 2-group is better understood by the equivalent notion of a crossed module, that we will define below. In fact, 2-groups were introduced originally as crossed modules in the 1950 's that later were recognized as being equivalent to 2-groupoids [60-62].

A 2-group $\mathcal{G}$ has the following elements:

(i) one object

(ii) morphisms

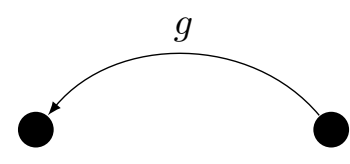

(iii) and 2-morphisms

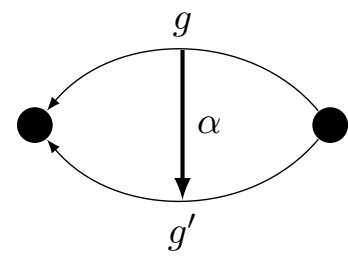

The same way the morphisms form a group under composition, as in Figure 2.8:

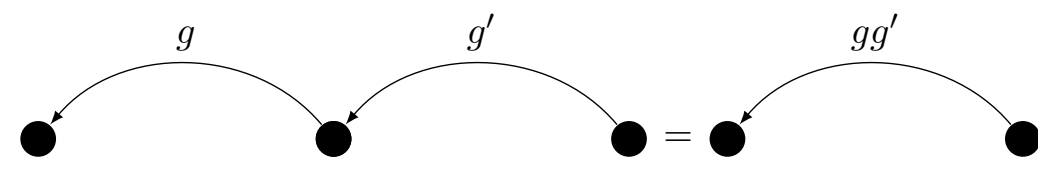

FIGURE 2.8: Composition of morphisms defining a group

The 2-morphisms form a group under horizontal composition, as in Figure 2.9:

as well as having a vertical

Vertical composition is also associative with identity and inverse.But the 2-morphisms do not form a group under this operation, because a given pair may not be composable: 


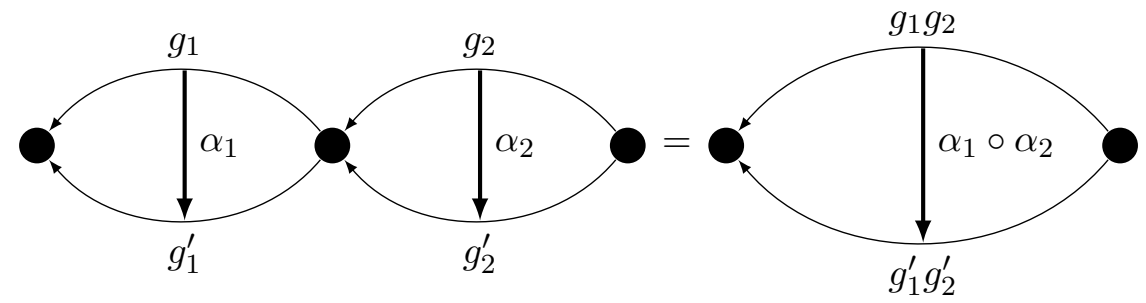

Figure 2.9: Horizontal composition of 2-morphisms

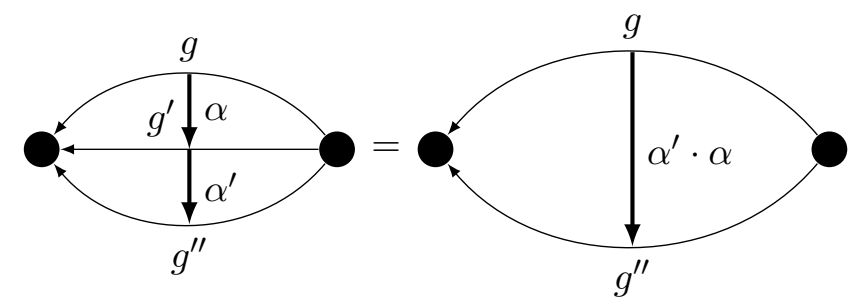

FIGURE 2.10: Vertical composition of 2-morphisms

their source and target may not match up. Also, horizontal and vertical composition are consistent between each other, meaning that the two ways one can read a composition diagram do not depend on which one is being done first.

As it was mentioned before, the concept of 2-groups and crossed modules are in oneto-one correspondence. Since crossed modules are easier to grasp, we embark on its description during the next paragraphs. We can construct a crossed module $(G, H, t, \alpha)$ from a 2- group as follows:

(1) Let $G$ be the set of morphisms in $\mathcal{G}$, made into a group with composition as the group operation, as in Figure 2.8.

(2) Let $H$ be the set of all 2-morphisms whose source is the identity

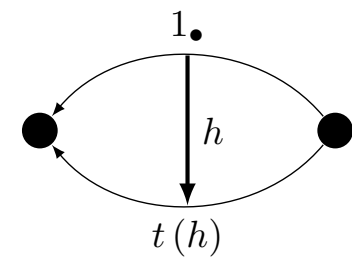

That can be turned into a using the horizontal composition as the group operation showed in Figure 2.11

(3) Hence, the latter equation suggests $t: H \rightarrow G$ to be a group homomorphism. This is, $t\left(h h^{\prime}\right)=t(h) t\left(h^{\prime}\right)$, for all $h \in H$. 


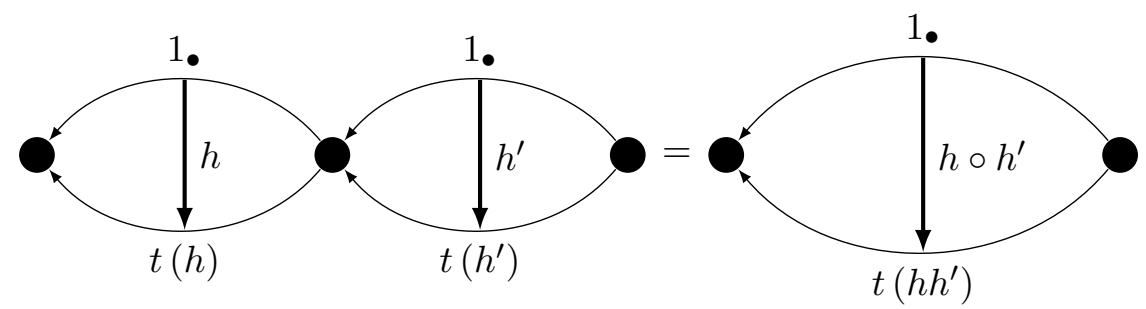

FIGURE 2.11: Group operation of horizontal composition of 2-groups

(4) The last element is an automorphism $\alpha: G \rightarrow$ Aut $(H)$, with the two natural operations:

$$
\alpha(g)\left(h h^{\prime}\right)=\alpha(g)(h) \alpha(g)\left(h^{\prime}\right) \quad \text { and } \quad \alpha\left(g g^{\prime}\right)(h)=\alpha(g)(h) \alpha\left(g^{\prime}\right)(h),
$$

for all $g, g^{\prime}$ in $G$ and $h, h^{\prime} \in H$. These are arranged as to make the following diagram consistent:

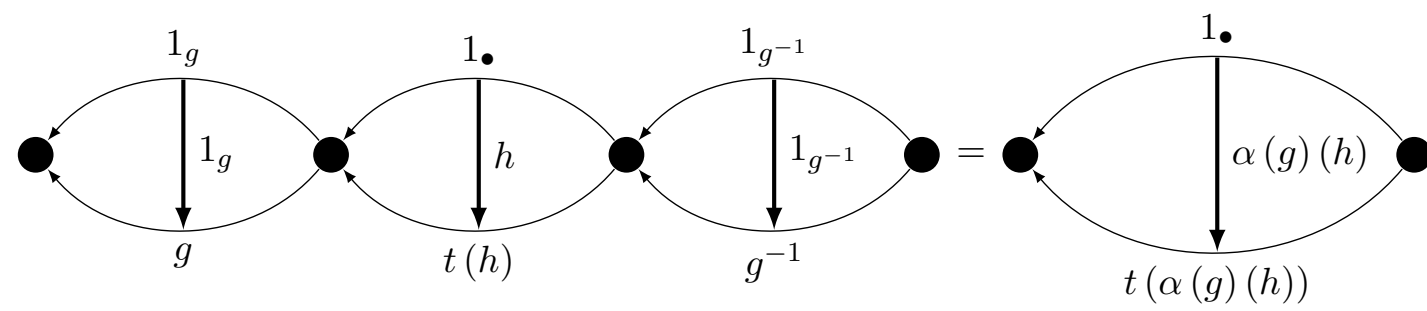

This is,

(5) For all $g \in G$ and $h \in H$, we need the action $\alpha$ to be $G$-equivariant

$$
t(\alpha(g) h)=g t(h) g^{-1},
$$

and

(6) For all $h, h^{\prime} \in H$, we need them to satisfy the Peiffer identity

$$
\alpha(t(h)) h^{\prime}=h h^{\prime} h^{-1}
$$

Where the Piffer identity is obtained by considering the following diagram where, over

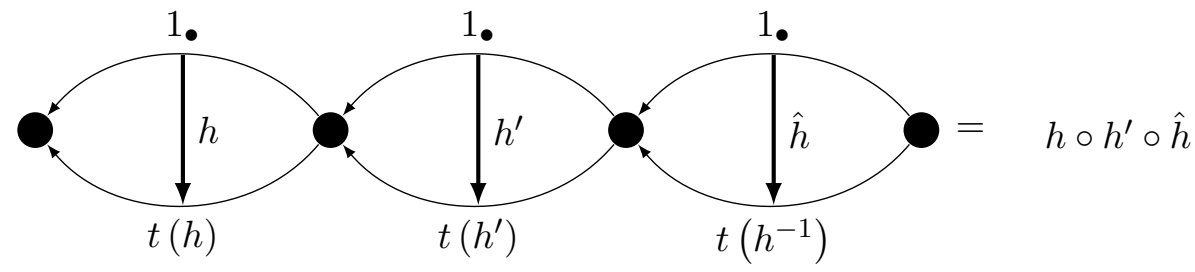


the horizontal composition, $\hat{h}$ stands for the horizontal inverse of $h$. The previous diagram can then be rewritten to in the following equivalent form:

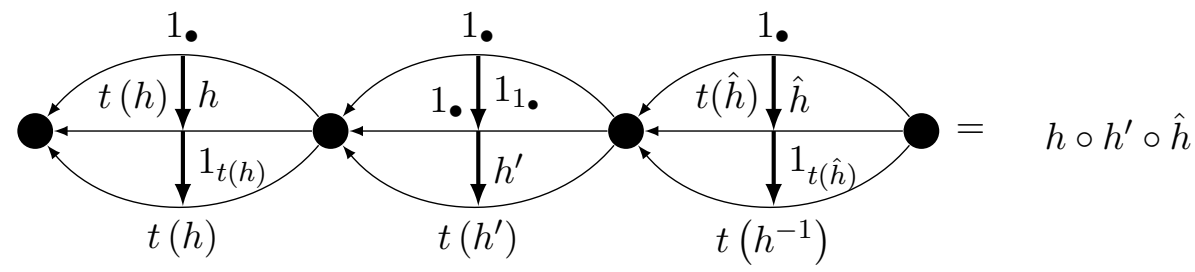

which, due to consistency must be unambiguously composed. Hence, by composing horizontally first we get:

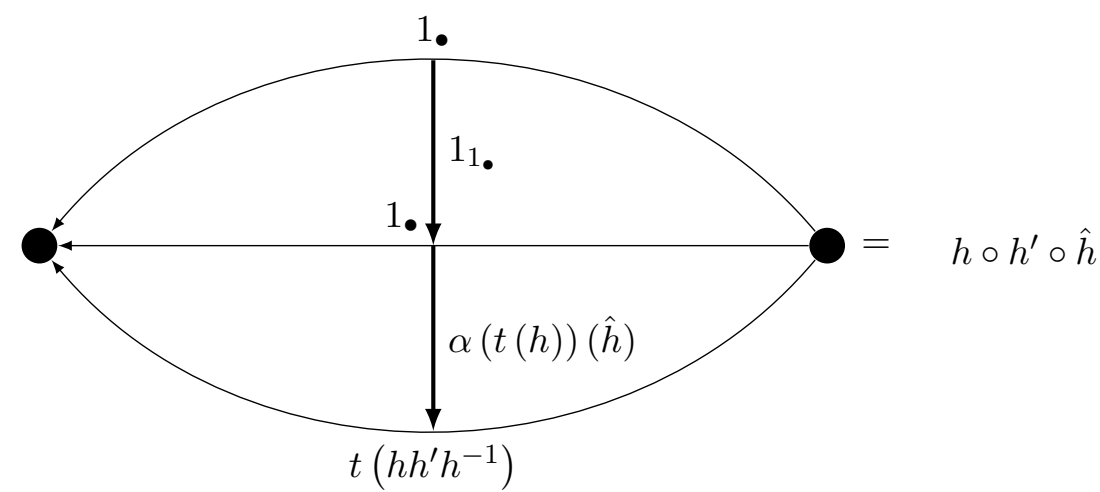

which give us the Peiffer identity when composing vertically. Summing up:

Definition 2.3.6 (Crossed modules). A crossed module is the tetrad $(G, H, t, \alpha)$, where $G$ and $H$ are groups, $t: H \rightarrow G$ is homomorphism and $\alpha: G \rightarrow \operatorname{Aut}(H)$ is an action, such that:

(i) $t(\alpha(g) h)=g(t(h)) g^{-1}$. This is, $t$ is $G$-equivariant for all $g \in G$ and $h \in H$, and

(ii) $\alpha(t(h)) h=h \circ h^{\prime} \circ \bar{h}$. This is, Peiffer identity holds for all $h, h^{\prime} \in H$.

The equivalence between a 2-group $\mathcal{G}$ and its crossed modulo $(G, H, t, \alpha)$ lies in realizing that 2-morphisms in $\mathcal{G}$ are the same as pairs $(g, h) \in G \times H$ defined as $(g, h): g \mapsto t(h) g$, given by the following 2-morphism diagram:

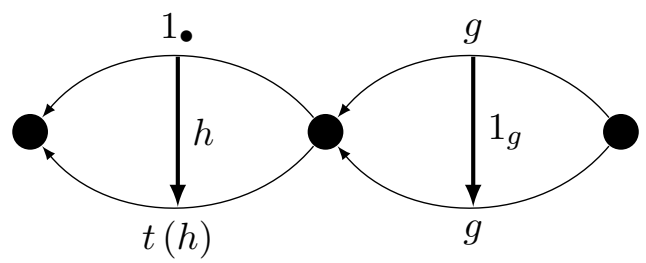


After this discussion, we formalize the equivalence between 2-groups and crossed modules with the following: theorem:

Theorem 2.3.7. Given a crossed module $(G, H, t, \alpha)$, there is a unique 2 -group $\mathcal{G}$ where:

(i) the group of morphisms is $G$,

(ii) a 2-morphism $\alpha: g \Rightarrow g^{\prime}$ is the same as the pair $(g, h) \in G \times H$ with $g^{\prime}=t(h) g$,

(iii) the vertical composite of $(g, h)$ and $\left(g^{\prime}, h^{\prime}\right)$, when they are composable, is given by

$$
(g, h) \cdot\left(g^{\prime}, h^{\prime}\right)=\left(g^{\prime}, h h^{\prime}\right)
$$

(iv) the horizontal composite of $(g, h)$ and $\left(g^{\prime}, h^{\prime}\right)$ is given by

$$
(g, h) \circ\left(g^{\prime}, h^{\prime}\right)=\left(g g^{\prime}, h \alpha(g)\left(h^{\prime}\right)\right),
$$

Conversely, given a 2-group $\mathcal{G}$, there is a unique crossed module $(G, H, t, \alpha)$ where:

(i) $G$ is the group of morphisms of $\mathcal{G}$,

(ii) $H$ is the group of 2-morphisms with source equal to 1. ,

(iii) $t: H \rightarrow G$ assigns to each 2-morphisms in $H$ its target,

(iv) the action $\alpha$ of $G$ on $H$ is given by

$$
\alpha(g) h=1_{g} \circ h \circ 1_{g^{-1}} .
$$

Hence, a Lie 2-group is understood to be a 2-group for which the groups $G$ and $H$ in its crossed module are Lie groups, with the maps $t: H \rightarrow G$ and $\alpha: G \rightarrow \operatorname{Aut}(H)$ being smooth, noticing that Aut $(H)$ is a Lie group in its own right. We stop here in order to discuss and criticize the latter exposition as a viable way to generalize gauge theories.

\subsection{Criticism of the $n$-group approach}

We have presented the fundamentals of gauge theory, first from a traditionally continuous perspective aiming towards a discrete version perspective that will be discussed in the next Chapter, along with some important examples. Also, we have shortly discussed the $n$-group program up to 2 -gauge. We can see that an immediate problem arises when generalizing from 1-gauge to 2-gauge dimensions, namely, the mathematical structures required to be satisfied are quite strongly tied: $\mathfrak{g}$-equivariancy and Peiffer identity. 
As for the modeling is concerned, several applications of the latter formalism are given in $[55,56]$. An interesting model has also been developed by our group, based on the topological invariant of Crane and Yetter [64] which is currently been peer reviewed. It is, however, the opinion of the author that the situation becomes even more cumbersome for 3-gauge, since further structure conditions, similar to those of Peiffer and $\mathfrak{g}$-equivariancy, appear. This situation undermines the flexibility of the models based on the $n$-group formalism approach. Therefore, there is a call for a different program aiming to generalizations. One of this possibilities has has been pursued in this work, with what we consider to be promising results, that we will pass on to describe over the next Chapters. 


\section{Chapter 3}

\section{The Toric Code and Quantum Double Models}

By now we expect to have introduced the most important concepts behind a gauge theory, in Chapter 2. We refer to:

(i) parallel transport,

(ii) curvature and

(iii) Wilson loops.

We will see that these have corresponding discrete analogs on its immediate discrete version, lattice gauge theory:

(i) parallel transport on a link,

(ii) holonomy and

(iii) state sum averaging,

respectively.

We will also present a generalization that have turn out to be quite successful at addressing and encompassing different physical attributes of a broad class of very interesting systems. Indeed, we present the poster child of lattice gauge theories, namely, the quantum double models (QDM), from which we drawn the inspiration for many of the features to be kept in the higher dimensional version presented later. 
We choose to go in a historical fashion, introducing an important particular case of the QDM: the Toric Code (TC). The Toric Code is known to be a 2-dimensional exactly solvable model that shows the main features of a topologically ordered system, this is, topologically dependent ground state degeneracy, anyonic excitations and non trivial statistics. Although Kitaev's TC appeared initially as a quantum error correcting code, it was soon realized the potential it had as a toy model to study its topologically ordered features, such as non-abelian statistics. For a good account of the QDM from a field theoretical point of view we refer the reader to $[65,66]$, however we are more concern with the practical construction that have led the research in recent years.

\subsection{Lattice gauge theory}

This discrete version is the basis of the models presented later and nearly all the ideas coming up from this formalism will have their analog in the models we will present over this and the following chapters. Let us now take the firsts steps towards a discretized version of a gauge theory. We will start by discretizing the manifold $M$ to construct what is known as a lattice. This construction is sufficient to account for the main features of a gauge theory and it is flexible enough to add more mathematical structure on top. For the sake of rigor, we will adopt a more formal approach on what follows.

Definition 3.1.1. A lattice $L$ is given by finite sets $\mathcal{V}, \mathcal{E}, \mathcal{F}$ as follows. the elements of the sets are called vertices, edges and faces respectively. There are two maps $\partial^{1}, \partial^{2}$, called boundary maps. The image of an element is called its boundary. $\partial^{1}$ assigns to each edge $e \in \mathcal{E}$ a set of two vertices $\left\{v_{1}, v_{2}\right\} \subseteq \mathcal{V}$. $\partial^{2}$ assigns to each face $f \in \mathcal{F}$ a sequence of edges $\left\{e_{1}, e_{2}, \cdots, e_{k}\right\} \subseteq \mathcal{E}$, determined up to cyclic permutations and up to reversal of the order, such that each edge $e_{i}$ has one vertex in common with the preceding edge $e_{i-1}$ and the other vertex in common with the following edge $e_{i+1}$.

Remark 3.1.2. In Appendix A, the decomposition of manifolds is treated by the related idea of Simplicial complexes as a generalization to higher dimensions $(n \geq 3)$.

Figure 3.1 depicts a triangulation of a section of a 2 - $d$ manifold in order to construct a lattice.

Another important concept is that of the orientation that we define in what follows

Definition 3.1.3. (1) Consider a lattice. If for an edge $e \in \mathcal{E}$ we distinguish between its boundary vertices by calling one initial vertex and the other final vertex, we call this an orientation $o_{e}$ of the edge. If all edges are oriented the lattice is called edge-oriented. 


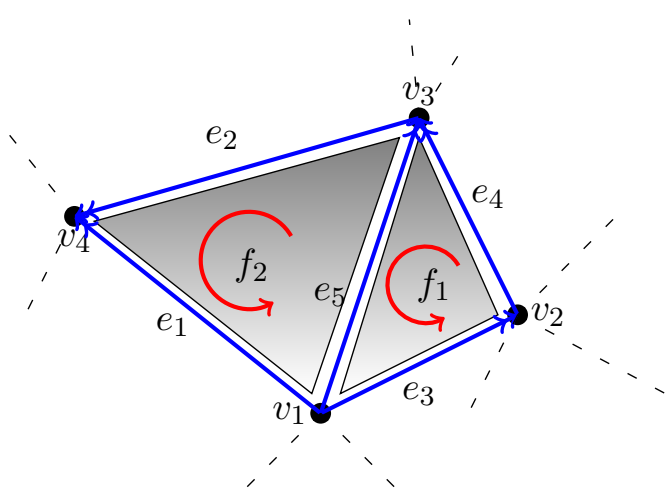

Figure 3.1: A section of a discretized 2- $d$ manifold into a lattice, Vertices $v_{i}$ are shown, as well as oriented edges $e_{i}$ and oriented faces $f_{i}$.

(2) Consider a lattice. If for a boundary of a face $\partial^{2} f$ the order of the corresponding edges $e_{i} \in \partial^{2} f$ is distinguished up to cyclic permutations, we call this an orientation $o_{f}$ of the face. If all faces are oriented the lattice is called face-oriented.

(3) If a lattice is both edge-oriented and face-oriented we simply call it oriented.

Now that the manifold has been characterized as an oriented lattice $L$, the next step is to define the relations between the continuous dynamical variables and its discrete analogs. We will begin by stating how to construct a discretized connection

- The assignment $\mathcal{E} \rightarrow G ; e \mapsto g_{e} ; \forall e \in \mathcal{E}, g_{e} \in G$. i.e. we assign a group element $g_{e}$ for each edge $e$ of the lattice. This represents the parallel transport of $A$ along $e$ in the direction from the initial vertex $v_{1}$ to the final vertex $v_{2}$.

- The assignment $\mathcal{V} \rightarrow G ; v \mapsto h_{v} ; \forall v \in \mathcal{V}, h_{v} \in G$. i.e. we assign a group element $h_{v}$ for each vertex $v$ of the lattice. This will modify the connection by transforming $g_{e}$ as

$$
g_{e}{ }^{\prime}=h_{v_{1}}^{-1} g_{e} h_{v_{2}}
$$

where $v_{1}$ is the initial vertex and $v_{2}$ is the final vertex. This is the discrete analogue of a gauge transformation.

The previous situation is exemplified on the figure 3.2.

Since we have defined the a discrete connection performing a parallel transport, we need now to define a discrete analog of the curvature. As we showed in the previous section, up to first order (and considering flat connections) we can think of the holonomy $g_{\partial \sigma}$ as to be a discrete version of the curvature 2-form F computed over a 2-cochain who's boundary is the closed loop over which the holonomy is being calculated (see equation 


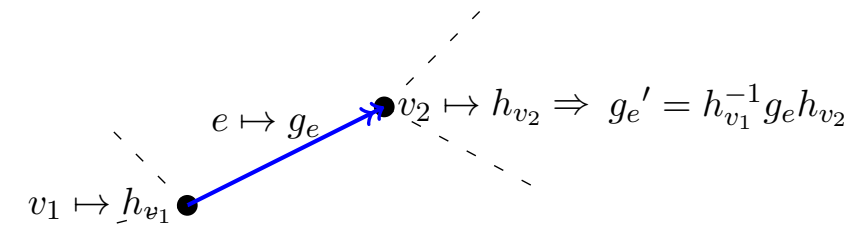

Figure 3.2: parallel transport $g_{e}$ and gauge transformation $g_{e}{ }^{\prime}$ over an edge $e$

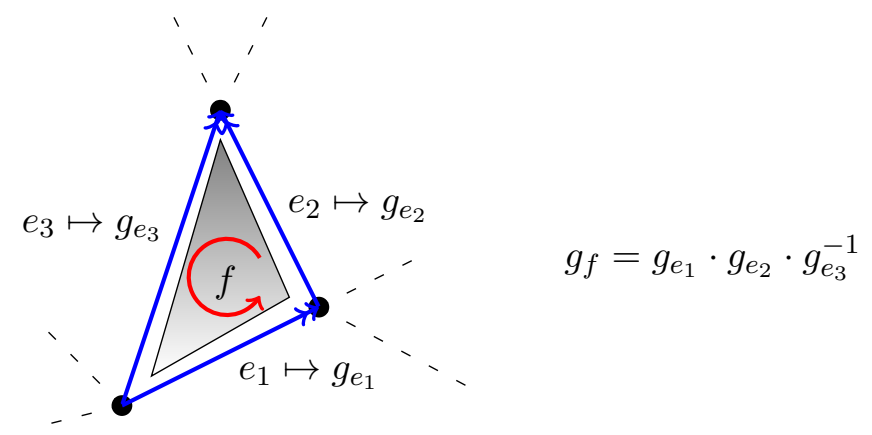

Figure 3.3: Holonomy $g_{f}$ of a triangular face $f$. The directions of the edges $e_{i}$ are depicted by the blue arrows while the direction of the face is depicted by the red circular arrow. Note that the only direction which is inconsistent between the two is $e_{3}$, hence the inverse element is taken.

(2.11)). Let us now consider a face $f \in \mathcal{F}$ with oriented edges that we will denote by $\left(e_{1}, e_{2}, \cdots, e_{k}\right)$, using the assignment previously defined we have $\left(e_{1}, e_{2}, \cdots, e_{k}\right) \mapsto$ $\left(g_{e_{1}}, g_{e_{2}}, \cdots, g_{e_{k}}\right)$ respectively. To compute the discrete holonomy we just multiply all the elements according to its relative orientation over the face as follows

$$
g_{f} \doteq g_{e_{1}}^{o_{1}} \cdot g_{e_{2}}^{o_{2}} \cdots g_{e_{k}}^{o_{k}}
$$

where $o_{i}= \pm 1$ depending on the relative orientation of the edge $e_{i}$ with the face $f$. In figure 3.3 the situation is exemplified for a triangular face.

What we have described is the appropriate notions of the discrete analogs of the dynamical variables of a pure Gauge Theory. The observables of the theory will be calculated using the the concept of Wilson loops already described. Let us then recall the path integral formulation of a pure gauge theory,

$$
\begin{aligned}
\mathcal{Z} & =\int_{M}\left[\mathcal{D} A_{i}\right] \exp \left[-\sigma_{i}\left(g_{\bar{\gamma}}^{(i)}\right)\right], \\
g_{\bar{\gamma}}^{(i)} & =-\mathcal{P} \exp \left(\int_{\bar{\gamma}} \mathbf{A}_{i}\right),
\end{aligned}
$$

where the measure $\left[\mathcal{D} A_{i}\right]$ is over the $i$-th 1 -form connection and $\bar{\gamma}$ is a closed loop. We have allowed for several connections to be present while a Wilson loop can be constructed 
from it. We also recall that this path integral stands for only a formal device and it is not necessarily well defined on its measure. On the other hand, as we already argued, the function $\sigma_{i}\left(g_{\gamma}^{(i)}\right)$ is a class function and henceforth also it will be it's exponential.

Let us now use the fact that the exponential of a class function is also a class function, to finally write

$$
\exp \left[-\sigma\left(g_{\gamma}\right)\right]=\sum_{V}(\operatorname{dim} V) \alpha_{V} \chi_{V}\left(g_{\gamma}\right)
$$

where we have expanded the class function $\exp \left[-\sigma\left(g_{\gamma}\right)\right]$ in characters of the irrep $\chi_{V}\left(g_{\gamma}\right), \alpha_{V}$ are the irrep coefficients of the expansion (see [68] for a comprehensive account on group theory and representations). The dimension of the finite vector space $V($ written $\operatorname{dim} V)$ is also included with later normalization purposes. More on this on later chapters.

In this discretization we should also consider the case of work with a possibly ill defined measure. The connection will be replaced by finite set of group variables playing the role of the parallel transport along edges of a lattice. Thus the natural integral over connections is the Haar measure at each edge. Schematically, the expression will be substituted for

$$
\int[\mathcal{D} A] \rightsquigarrow \int \prod_{e} d g_{e}
$$

where we have adjusted the latter so it can be written in terms of the lattice's elements.

Putting all together by using equations (3.4) and (3.5), the path integral equation (3.3) takes the discretized general form

$$
\mathcal{Z} \rightsquigarrow \mathcal{Z}_{L}=\int \prod_{e} d g_{e} \sum_{V_{f}}\left(\prod_{f} \operatorname{dim} V_{f} \alpha_{V_{f}}\right) \prod_{f} \chi_{V_{f}}\left(g_{f}\right) .
$$

Remark 3.1.4. We expect this to be a finite integral since it is an integral of a product of bounded functions over a compact space.

\subsubsection{Observables, Wilson Loops and Gauge Fixing}

Let us allow the coefficients $\alpha_{V}$ of equation (3.6) to be dependent of both representations: the vector space $V$ and faces $f$. We can rewrite this expression such that the summation over the representations go to the front. Again, we are assuming this expression to be convergent by representing an integral of a product of bounded functions over a compact 
space. So the partition function $\mathcal{Z}_{L}$ reads

$$
\mathcal{Z}_{L}=\sum_{V_{f}}\left(\prod_{f} \operatorname{dim} V_{f} \alpha_{V_{f}, f}\right) \int \prod_{e} d g_{e} \prod_{f} \chi_{V_{f}}\left(g_{f}\right),
$$

expression that is known as state sum. Furthermore, let us define the following quantity

$$
\mathcal{W}_{L}\left(V_{f}, o_{f}, g_{e}, o_{e}\right) \doteq \int \prod_{e} d g_{e} \prod_{f} \chi_{V_{f}}\left(g_{f}\right)
$$

where $o_{f}$ stands for face orientation and $o_{e}$ stands for edge orientation. Using (3.8), equation (3.7) can finally stated as

$$
\mathcal{Z}_{L}=\sum_{V_{f}}\left(\prod_{f} \operatorname{dim} V_{f} \alpha_{V_{f}, f}\right) \mathcal{W}_{L}\left(V_{f}, o_{f}, g_{e}, o_{e}\right)
$$

Remark 3.1.5. Constructing a lattice gauge theory will ultimately be related to make sense of the defined term (3.8) and have a clear algorithm on how to calculate it's value.

As it was argued on the previous section, observables are linked to Wilson loops $\left(\gamma_{W}\right)$ and it's expectation value is readily calculated by the standard formula

$$
\left\langle\gamma_{W}\right\rangle_{L} \doteq \frac{\mathcal{Z}_{L}\left[\bar{\gamma}_{W}\right]}{\mathcal{Z}_{L}}
$$

where

$$
\mathcal{Z}_{L}\left[\bar{\gamma}_{W}\right] \doteq \sum_{V_{f}}\left(\prod_{f} \operatorname{dim} V_{f} \alpha_{V_{f}, f}\right) \mathcal{W}_{L}\left[\bar{\gamma}_{W}\right]\left(V_{f}, o_{f}, g_{e}, o_{e}\right)
$$

and

$$
\mathcal{W}_{L}\left[\bar{\gamma}_{W}\right]\left(V_{f}, o_{f}, g_{e}, o_{e}\right) \doteq \int \prod_{e} d g_{e} \chi_{W}\left(g_{\bar{\gamma}}\right) \prod_{f} \chi_{V_{f}}\left(g_{f}\right) .
$$

In equation (3.12) the term $\chi_{W}\left(g_{\bar{\gamma}}\right)$ is the character of the irrep of the holonomy over the closed loop $\bar{\gamma}$ related to the Wilson observable. In general, the partition function (3.11) has the property that certain group of integral may be removed without changing its value. The corresponding group element is just set to some fixed value, usually the neutral element. In lattice gauge theory this is known as gauge fixing, since one may think of this as fixing unphysical degrees of freedom in the representation of the connection.

On the other hand, certain local operations over the lattice $L$ may not change the value of some factors of the equation (3.11). This fact will be extremely important when the topological features of the gauge theory are taken into consideration. We will define 
the following operation that, under certain conditions, does not change the value of the partition function:

Definition 3.1.6. Let $L$ be a lattice and $e$ an edge in $L$ which is bound by two distinct vertices $v_{1}, v_{2}$. Then we define a new lattice $L^{\prime}$ as follows. The vertices of $L^{\prime}$ are the vertices of $L$ except for $v_{1}$ and $v_{2}$ which are replaced by a single vertex $v$. The edges of $L^{\prime}$ are the edges of $L$ except for $e$. The faces of $L^{\prime}$ are the faces of $L$. The boundary maps of $L^{\prime}$ are those of $L$, except that $e$ is removed from boundaries of faces and $v_{1}$ and $v_{2}$ are replaced with $v$ in boundaries of edges. The transition from $L$ to $L^{\prime}$ is called a fusion of vertices. The situation is depicted in figure (3.4)
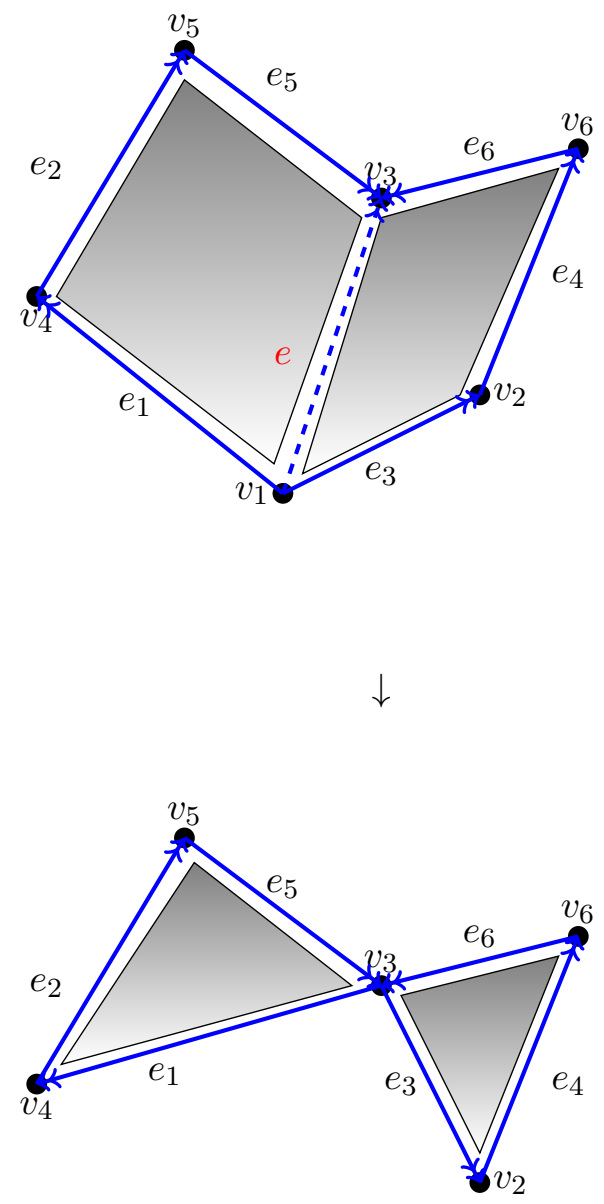

FiguRE 3.4: A fusion of vertices, the local operation depicted has absorbed the edge $e$ without changing the value of the partition function by setting its group element to the identity .

The following proposition is derived from the last procedure:

Proposition 3.1.7. Let $L$ be a lattice and e be an angle in $L$ whose initial and final vertex are distinct. Let $L^{\prime}$ be a lattice obtained by removing e through a fusion move. If $\mathcal{W}_{L}\left(V_{f}, o_{f}, g_{e}, o_{e}\right)=\mathcal{W}_{L}\left(V_{f}, o_{f}\right)$, then $\mathcal{W}_{L}\left(V_{f}, o_{f}\right)=\mathcal{W}_{L^{\prime}}\left(V_{f}, o_{f}\right)$ for any labeling $V_{f}$ of 
faces with representations. In particular, the partition functions are equal $\mathcal{Z}_{L}=\mathcal{Z}_{L^{\prime}}$, if well defined.

Proof. The proof is by construction and straightforward. Setting the target edge $e$ to the identity will not change the value of the factor $\mathcal{W}_{L}\left(V_{f}, o_{f}\right)$, regarded the orientation is still respected. It follows that, this proviso, $\mathcal{Z}_{L}=\mathcal{Z}_{L^{\prime}}$ where $L$ and $L^{\prime}$ are related through vertex fusion moves.

The following corollary is derived from the above:

Corollary 3.1.8. Integrals in (3.7) can be removed without changing the partition function, as long as the set of edges for which this is done does not contain any closed loop

Proof. The vertex fusion move allows to repeat this procedure indefinitely as long as the vertices in question don't coincide, the latter is excluded by not allowing closed paths under this operation. By construction we have $\mathcal{Z}_{L}=\mathcal{Z}_{L^{\prime}}$ where $L$ and $L^{\prime}$ are related through finite vertex fusion moves.

\subsection{Quantum Double models overview}

We insist that the construction shown on the previous section goes beyond the mere discretization of a continuous gauge theory. In fact, it allows us to construct a lattice gauge theory from the start by using generalized classes functions as weight actions. This means that we are not necessarily expecting a certain lattice gauge model to be a discretized version of a continuous one. Hence, we have freedom to create and study new toy models with certain physical and/or mathematical characteristics that we can explore. The step of upgrading to more dimensions can be easily made by considering a simplicial complex, or more generally a $C W$-complex instead of a lattice, carrying with them all the topological machinery of singular homology and related techniques. We refer the reader to Appendix B for a review of these ideas.

From a historical perspective, and even though we do not describe enroll on their description, we mention can mention other topological quantum gauge theories that have been introduced. Among them, the Levin-Wen model [23], which is considered to be a generalization of the quantum double model for unitary tensor categories, and henceforth, provides a general recipe to translate mathematical objects into physical concepts and vice versa. 


\subsubsection{The toric code}

Consider a planar (ordered) square lattice (see figure 3.5). The order has been depicted as arrows on the black links of the physical lattice. In this case (although irrelevant due to the gauge group in question) we have considered a direction from left to right to each horizontal link, and from bottom to top for each vertical link. Now, proceed to associate to each link a Hilbert space with basis elements indexed by $\mathbb{Z}_{2}$. Physically, this is still a spin model with two degrees of freedom on each link.

The lattice can also be indexed by a group of links converging into a vertex $v \in \mathcal{V}$ (see figure 3.5), where $\mathcal{V}$ is the set of vertex of the lattice. Similarly, the lattice can also be indexed by by a group of links forming a plaquette $p \in \mathcal{P}$, where $\mathcal{P}$ is the set of plaquettes on the lattice. with this rearrangement, the TC is thought as being a dynamical model and the Hamiltonian is a summation of vertex and plaquette operators in the following way

$$
H_{T C}=-\sum_{v \in \mathcal{V}} A_{v}-\sum_{p \in \mathcal{P}} B_{p}
$$

where the operators $A_{v}$ and $B_{p}$ are defined in terms of the $\sigma_{x}$ and $\sigma_{z}$ Pauli matrices (see $[24,27]$ for more details). These operators are constructed in a way that they commute $\forall v, p$ in the lattice.

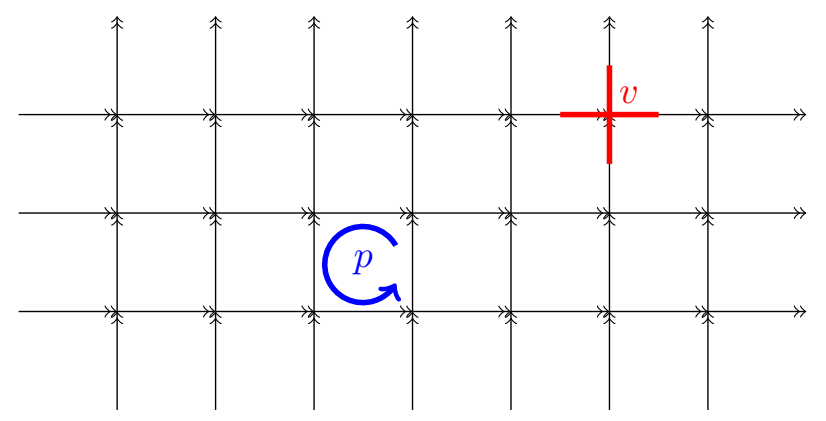

Figure 3.5: A square ordered lattice, vertex $(v)$ and plaquette $(p)$ sites

Moving on to briefly describe the elementary excitations of the system (see figure 3.6), we can say that they correspond to certain chains of $\sigma_{x}$ and $\sigma_{z}$ operators (ribbon operators) which create two quasi-particles at the end points of the ribbon. A string of $\sigma_{x}$ operators gives magnetic charges (called fluxions), denoted by $m$, and a chain of $\sigma_{z}$ operators gives electric charges (called chargeons), denoted by $e$. Moreover, the movement of quasiparticles is equivalent to extending the corresponding ribbons. Such an extension is used to study their braidings properties and statistic.

Similarly, the braiding can also be constructed by simultaneous application of $\sigma_{x}$ and $\sigma_{z}$ chains, i.e. the fusion of $m$ and $e$ denoted $\epsilon:=m \otimes e$. These three particles together with 


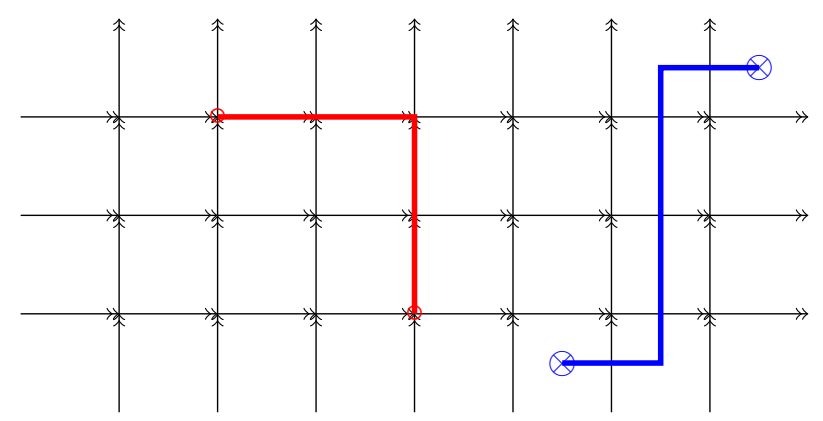

FIGURE 3.6: Elementary excitations: charge (०) living on the verteces, and flux $(\otimes)$ living on the plaquettes

the vacuum give the system of anyons corresponding to the group $\mathbb{Z}_{2}$; they are indeed the four irreducible representations of the quantum double of $\mathbb{Z}_{2}$, denoted by $D\left(\mathbb{Z}_{2}\right)$.

\subsubsection{The algebra: The Drinfeld double of a finite group}

Behind the QDM there is a rich mathematical structure. In fact, the QDM get their name from the Drinfeld Double algebraic structure. We will review this algebraic duality succintively, mainly from the mathematical standpoint, we will later elaborate on its physical realizations. In what follows we will heavily follow [69].

\section{Notation.}

- We will denote by $\mathbb{C}$ the set of complex numbers and $\mathbb{C}^{x}$ its multiplicative group.

- We will denote by $x^{*}$ the complex conjugate of $x \in \mathbb{C}$ and $|x|^{2}=x x^{*}$.

- The identity element of a group $G$ will be denoted by $e$.

- For a subgroup $K$ of $G$ and $g \in K, Z_{K}(g)$ denotes the centralizer of $g$ in $K$ : $Z_{K}(g)=\{h \in K: h g=g h\}$.

- We will write $g \sim^{K} g^{\prime}$ if there exists $h \in K$ such that $h g h^{-1}=g^{\prime}$ (dropping the $K$ when not required).

- The conjugacy class of $g \in G$ will be denoted by $\bar{g}$, i.e. $\bar{g}=\{h g h-1: h G\}$.

- The representations of the group are considered always to be over complex numbers, and for a representation $\rho$ of a group, $\rho^{*}$ is its complex conjugate representation.

- The character of $\rho$ is denoted by $\chi(\cdot, \rho)=\operatorname{tr}_{\rho}(\cdot)$, and $\mathbf{1}$ will be the trivial representation, i.e. $\operatorname{tr}_{\mathbf{1}}(\cdot)=\mathbf{1}$. 
- $\delta$ denotes the Kronecker delta function and for any relation $p, \delta_{p}=1$ if $p$ holds and otherwise $\delta_{p}=0$.

- The size of a set $X$ is denoted by $|X|$.

- Finally, equivalence of categories, and isomorphism of groups and representations are shown by $\simeq$.

Definition 3.2.1 (Quantum Double). Consider now a finite group $G$. The quantum double or Drinfeld double of $G$ denoted by $D(G)$, is a Hopf algebra containing $\mathbb{C}(G)$. $D(G)$ can be described by the $\mathbb{C}$-basis $\left\{g h^{*}: g, h \in G\right\}$ and the operations of the Hopf algebra $\delta, m, \mathcal{S}$ (see table 3.1).

\begin{tabular}{|c|c|}
\hline Operation & Action over a $\mathbb{C}$-basis $\left(\right.$ with $g=\sum_{h} g h^{*}$ and $\left.h^{*}=e h^{*}\right)$ \\
\hline product & $m\left(g_{1} h_{1}^{*}, g_{2} h_{2}^{*}\right)=\delta_{h_{2}, g_{2}^{-1} h_{1} g_{2}}\left(g_{1} \cdot g_{2}\right) h_{2}^{*}$ \\
co-product & $\delta\left(g h^{*}\right)=\sum_{h_{1} \cdot h_{2}=h} g h_{1}^{*} \otimes g h_{2}^{*}$ \\
identity & $\eta=\sum_{h} e h^{*}$ \\
co-Identity & $\varepsilon\left(g h^{*}\right)=\delta_{h, e}$ \\
Antipode & $\mathcal{S}\left(g h^{*}\right)=g^{-1}\left(g h^{-1} g^{-1}\right)^{*}$ \\
\hline
\end{tabular}

TABLE 3.1: Operations over a quantum double with $\mathbb{C}$-basis $\left\{g h^{*}: g, h \in G\right\}$

As for what the representation of this group is concern, a general reference to be consulted is [68]. Consider an element $a \in G$ and let $\pi$ be a representation of $Z(a)$ over the vector space $W$ encompassing the basis $\left\{w_{1}, \cdots, w_{d}\right\}$. Define the vector space $V_{(\bar{a}, \pi)}$ with basis $\left\{\left|b, w_{i}\right\rangle: b \in a, 1 \leq i \leq d\right\} . V_{(\bar{a}, \pi)}$ is a representation of $D(G)$ as follows. For any $b \in \bar{a}$ fix $k_{b} \in G$ such that $b=k_{b} a k_{b}^{-1}$. (Let $k_{a}=e$.) Observe that $k_{g b g^{-1}}^{-1} g k_{b}$ is always in $Z(a)$, and then for any $w \in W, b \in \bar{a}$, and $g h^{*} \in D(G)$ define

$$
g h^{*}|b, w\rangle=\delta_{h, b}\left|g b g^{-1}, \pi\left(k_{g b g^{-1}}^{-1} g k_{b}\right) w\right\rangle
$$

which gives a representation of $D(G)$. On the other hand, the character of this representation $\chi(\bar{a}, \pi)$ has the form

$$
\chi_{(\bar{a}, \pi)}\left(g h^{*}\right)=\delta_{h \in \bar{a}} \delta_{g h, h g} \operatorname{tr}_{\pi}\left(k_{h}^{-1} g k_{h}\right) .
$$


If $\pi$ is an irreducible representation (irrep) of $Z(a)$, then the representation $V_{(\bar{a}, \pi)}$ of $D(G)$ is irreducible as well. Conversely, all irreps of $D(G)$ are of the above form and are indexed by conjugacy classes of $G$ and irreps of the centralizer of a fixed element in the corresponding conjugacy class.

The trivial representation of $D(G)$ is indexed by $\mathbf{0}=(e, \mathbf{1})$. Moreover, the (charge) conjugation of $(\bar{a}, \pi)$, which, following [69], is denoted by $(\bar{a}, \pi)^{v}$, is isomorphic to $\left(a^{-1}, \pi^{*}\right)$. The conjugacy class $\bar{a}$ of an irrep $(\bar{a}, \pi)$ is called its magnetic charge and $\pi$ is its electric charge. $(\bar{a}, \pi)$ is called a chargeon if $a=e$ and a fluxion if $\pi=\mathbf{1}$.

Irreducible representations of $D(G)$ are orthogonal to each other with respect to the following inner product

$$
\left\langle\chi_{1}, \chi_{2}\right\rangle=\frac{1}{|G|} \sum_{g, h}\left(\chi_{1}\left(g h^{*}\right), \chi_{2}\left(g h^{*}\right)\right),
$$

then the multiplicity of the irrep $(\bar{a}, \pi)$ in the character $\chi$ is equal to $\left\langle\chi_{(\bar{a}, \pi)}, \chi\right\rangle$.

Definition 3.2.2 (Fusion rules). Let $(\bar{a}, \pi)$ and $\left(\overline{a^{\prime}}, \bar{\pi}^{\prime}\right)$ be two irreps of $D(G)$. Then using the co-product (see table 3.1$),(\bar{a}, \pi) \otimes\left(\bar{a}^{\prime}, \pi^{\prime}\right)$ is also a representation of $D(G)$, since in a base $v \otimes w$ given by $\delta\left(g h^{*}\right) v \otimes w$, that is isomorphic to the direct sum of irreducible ones:

$$
(\bar{a}, \pi) \otimes\left(\overline{a^{\prime}}, \pi^{\prime}\right)=\bigoplus_{(\bar{h}, \rho)} N_{(\bar{a}, \pi)\left(\overline{\left.a^{\prime}, \pi^{\prime}\right)}\right.}^{(\bar{h}, \rho)}(\bar{h}, \rho)
$$

where $N_{(\bar{a}, \pi)\left(\overline{\left.a^{\prime}, \pi^{\prime}\right)}\right.}^{\left(\bar{h},{ }^{\prime}\right.}(\bar{h}, \rho)$ is a non-negative integer.

In fact, in [69], it is said that the aforementioned numbers may be computed by the use of the Verlinde formula in the following way: define the matrix $S$ whose rows and columns are indexed by irreps of $D(G)$ and

$$
S_{(\bar{a}, \pi)\left(\bar{a}^{\prime}, \pi^{\prime}\right)}=\frac{1}{|Z(a)| \cdot\left|Z\left(a^{\prime}\right)\right|} \sum_{h: h a^{\prime} h^{-1} \in Z(a)} \operatorname{tr}_{\pi}\left(h a^{\prime-1} h^{-1}\right) \operatorname{tr}_{\pi^{\prime}}\left(h^{-1} a^{-1} h\right),
$$

then the $N_{X Y}^{Z}$ are computed in terms of $S$ as

$$
N_{X Y}^{Z}=\sum_{U} \frac{S_{X U} S_{Y U} S_{Z U}^{*}}{S_{0 U}}
$$

where the summation runs over all irreps $U$, and $\mathbf{0}=(e, \mathbf{1})$ is the trivial representation. To link these topics more strongly to what we presented on the previous chapter, we will denote by $\mathcal{Z}(G)$ the category of finite dimensional representations of $D(G)$ over the complex numbers. Hence, every object of $\mathcal{Z}(G)$ is isomorphic to a direct sum of simple 
objects, i.e., irreducible representations. In this context, $\mathcal{Z}(G)$ is braided monoidal category also interpreted as a fusion category [70], where the fusion rules are given by the Verlinde formula (eq. 3.19). Moreover, the R-matrix in this case is given by

$$
R=\sum_{g \in G} g^{*} \otimes g
$$

that defines the braiding $C_{X, Y}=P R: X \otimes Y \rightarrow Y \otimes X$ of two representations $X$ and $Y$, where $P$ is the transposition of $X$ and $Y$, i.e. $C_{X, Y} v \otimes w=\sum_{g} g w \otimes g^{*} v$. Also, $\mathcal{Z}(G)$ is a modular tensor category [71].

\subsubsection{The quantum double model formalism}

We are now in position to discuss the main ingredients of the QDM. We recall that the famous TC model can be considered as a QD representation of the group $\mathbb{Z}_{2}$. To introduce the dynamic of the model we will consider a natural generalization of the Kitaev Hamiltonian (3.13) to include finite groups. As it was the case with the TC, consider a planar lattice with oriented links (edges). We associate to each link $l$ of the lattice $\mathcal{L}$ the Hilbert space $\mathcal{H}_{l}:=\mathbb{C}(G)$ with orthonormal basis $\{|g\rangle: g \in G\}$ where now $G$ is a finite group. Hence, the full Hilbert space of the model is

$$
\mathcal{H}:=\bigotimes_{l \in \mathcal{L}} \mathcal{H}_{l}
$$

We assume that the direction of a link can be reversed (see figure 3.7) changing the basis vector corresponding from $|g\rangle$ to $\left|g^{-1}\right\rangle$ (and extending linearly). Physically, this will account for the process of parallel transport on a regular gauge theory.

$$
\left\{|g\rangle=\left\{\left|g^{-1}\right\rangle\right.\right.
$$

FiguRE 3.7: Oriented link with proper basis element $|g\rangle$ associated to it, equivalence to its inverse element $\left|g^{-1}\right\rangle$ and the inversion of the link orientation

A pair $s=(v, p)$ of adjacent vertex $v$ and plaquette $p$ is referred to as a site. For any these sites $s=(v, p)$ we define the operators $A_{s}^{g}$ and $B_{s}^{h}, g, h \in G$ which are depicted in figure 3.8. In this figure we are depicting the elements of the lattice in an obvious way. The operators act directly over the converging links of a vertex $v$ (in the case of $A_{s}^{g}$ ) and over the links constituting a plaquette $p$ (in the case of $B_{s}^{h}$ ) 

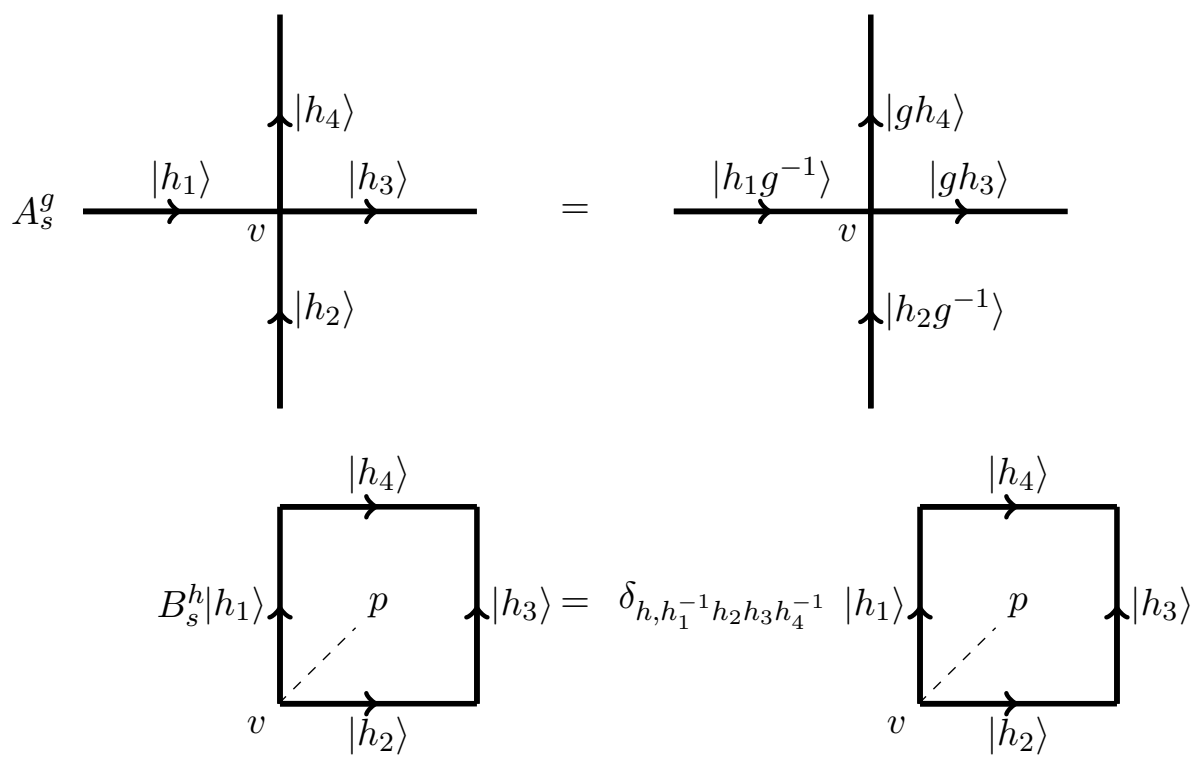

Figure 3.8: Definition of the operators for a QDM, on a vertex $\left(A_{s}^{g}\right)$ and on a plaquette $\left(B_{s}^{h}\right)$. Note that if $h$ is in the center of $G, B_{s}^{h}$ depends only on the plaquette $p$ and not on the vertex $v$.

Note that $A_{s}^{g}$ depends only on the vertex $v$ and not on $p$, therefore we can write $A_{v}^{g}$ instead of $A_{s}^{g}$. On the other hand, if $h$ is in the center of $G, B_{s}^{h}$ become independent of $v$, so we can write $B_{p}^{h}$ instead of $B_{s}^{h}$, and are called the vertex and plaquette operators, respectively.

The following relations are fulfilled by the previous operators:

$$
\begin{aligned}
A_{s}^{g} A_{s}^{g^{\prime}} & =A_{s}^{g g^{\prime}}, \\
\left(A_{s}^{g}\right)^{\dagger} & =A_{s}^{g^{-1}}, \\
B_{s}^{h} B_{s}^{h^{\prime}} & =\delta_{h, h^{\prime}} B_{s}^{h} \\
\left(B_{s}^{h}\right)^{\dagger} & =B_{s}^{h} \\
A_{s}^{g} B_{s}^{h} & =B_{s}^{g h g^{-1}} A_{s}^{g},
\end{aligned}
$$

which can be obtained by an explicit calculation using the definitions of figure 3.8 .

Remark 3.2.3. The set of equations (3.22) allows us to conclude that $g h^{*} \mapsto A_{s}^{g} B_{s}^{h}$ is an isomorphism between the quantum double $D(G)$ and the algebra of operators acting on the site $s[69]$. 
The situation for different sites $\left(s \neq s^{\prime}\right)$ is as follows

$$
\begin{aligned}
{\left[A_{s}^{g}, A_{s^{\prime}}^{g^{\prime}}\right] } & =0 \\
{\left[A_{s}^{g}, B_{s^{\prime}}^{h^{\prime}}\right] } & =0 \\
{\left[B_{s}^{h}, B_{s^{\prime}}^{h^{\prime}}\right] } & =0
\end{aligned}
$$

this means, these operators naturally commute. By defining

$$
\begin{aligned}
A_{v} & :=A_{s} \\
& :=\frac{1}{|G|} \sum_{g \in G} A_{s}^{g},
\end{aligned}
$$

and

$$
\begin{aligned}
B_{p} & :=B_{s} \\
& :=B_{s}^{e},
\end{aligned}
$$

we can construct the Hamiltonian

$$
H_{Q D}=-\sum_{v} A_{v}-\sum_{p} B_{p}
$$

where we have used equations (3.24) and (3.25), the summations run over all vertices $v$ and plaquettes $p$. The latter is known as Kitaev's Hamiltonian. Note now that, thus defined the operators $A_{v}$ and $B_{p}$ (eq (3.24) and (3.25), respectively), we have the following commutation relation between them

$$
\left[A_{v}, B_{p}\right]=0, \quad \forall v, p
$$

which can be easily proven by the use of the set of algebra equations (3.22) and (3.23). Remark 3.2.4. By the aim of equation (3.27) Kitaev's Hamiltonian is completely soluble. Furthermore, the ground state of $H_{Q D}$ is a state $\left|\psi_{0}\right\rangle$ such that

$$
\begin{aligned}
& A_{v}\left|\psi_{0}\right\rangle=\left|\psi_{0}\right\rangle, \\
& B_{p}\left|\psi_{0}\right\rangle=\left|\psi_{0}\right\rangle,
\end{aligned}
$$

simultaneously.

Remark 3.2.5. For a planar lattice, the ground state is unique and can be explicitly computed [24]. 
Moreover, given any state $|\psi\rangle \in \mathcal{H}$ the subspace $\mathcal{H}_{0} \subset \mathcal{H}$ of the ground state (considering degeneracy) can be given by following projector operator $P_{0}: \mathcal{H} \rightarrow \mathcal{H}_{0}$

$$
P_{0} \doteq \prod_{v} A_{v} \prod_{p} B_{p}
$$

Let us focus on a single link $l$ of the lattice $\mathcal{L}$ since they naturally index the entire lattice. As it was stressed before, to a link $l$ we associate a Hilbert space $\mathcal{H}_{l}=\mathbb{C}(G)$ with orthonormal $\mathbb{C}$-basis $\{|g\rangle: g \in G\}$. We can define the local operators shown in table 3.2 .

\begin{tabular}{|c|c|}
\hline Operator & Definitions \\
\hline Left shift (left product) & $L_{l}^{g}|h\rangle \doteq|g h\rangle$ \\
Right shift (right product) & $R_{l}^{g}|h\rangle \doteq|h g\rangle$ \\
Projection & $T_{l}^{g}|h\rangle \doteq \delta_{g, h}|h\rangle$ \\
\hline
\end{tabular}

TABLE 3.2: Local operators acting on a link $l, g, h \in G$

The algebra of these local operators for all $g, h \in G$ and $l \in \mathcal{L}$ is

$$
\begin{aligned}
L_{l}^{g} L_{l}^{h} & =L_{l}^{g h}, \\
R_{l}^{g} R_{l}^{h} & =R_{l}^{h g}, \\
T_{l}^{g} T_{l}^{h} & =\delta_{g, h} T_{l}^{g}, \\
L_{l}^{g} T_{l}^{h} & =T_{l}^{g h} L_{l}^{g}, \\
R_{l}^{g} T_{l}^{h} & =T_{l}^{h g} R_{l}^{g}, \\
L_{l}^{g} R_{l}^{h} & =R_{l}^{h} L_{l}^{h} .
\end{aligned}
$$

Note that the notion of the parallel transport can be implemented in a similar fashion than it was done in figure 3.7. The situation is depicted in figure 3.9 using the local operators $L_{l}^{g}$ and $R_{l}^{g}$.

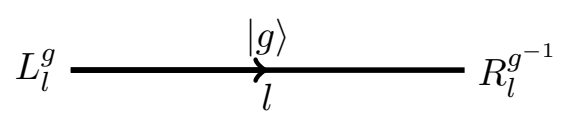

FiguRE 3.9: Equivalence on a parallel transport for the left and right shift operators

Consider now the cells depicted in figure 3.10 as a reference for indexing the links in a one to one correspondence. With a certain abuse of notation we are using the element 
of the group $h_{i}$ to index the links directly. Using the shift and projector operators (see table 3.2) the quantum double operators $A_{s}^{g}$ and $B_{s}^{h}$ can be constructed as follows

$$
\begin{aligned}
& A_{s}^{g}=R_{h_{1}}^{g^{-1}} \otimes R_{h_{2}}^{g^{-1}} \otimes L_{h_{3}}^{g} \otimes L_{h_{4}}^{g},
\end{aligned}
$$

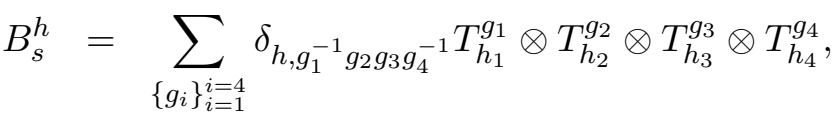

which can be easily proven to yield the same algebra (3.22) for the aforementioned operators.

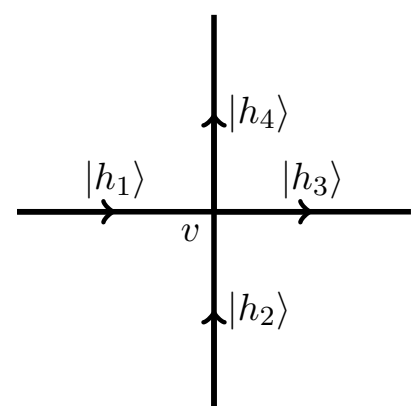

a)

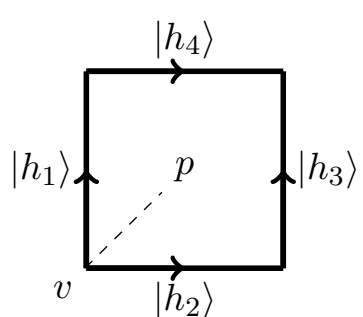

b)

Figure 3.10: a) Reference cell for the action of the vertex operator $A_{s}^{g}$. b) Reference cell for the action of the a plaquette operator $B_{s}^{h}$ in terms of local shift and projector operators

\subsubsection{Ribbon operators and elementary excitations}

This subsection is presented out of completeness. We remind the reader that although valid in two dimensions, these techniques are not well translated to higher dimensions. Although the present work is not focusing on understanding the excited states of the models, the present techniques may be of help when studying the region of the energy spectrum. The basic idea is to define a new kind of operators that will ultimately measure the excitations of quantum double models and their exotic statistic.

Definition 3.2.6 (Ribbon). A ribbon $\xi$ on the lattice is a sequence of adjacent sites connecting two sites $s_{0}$ and $s_{1}$ as shown in figure 3.11. Where we assume that $s_{0}$ is the starting site of the ribbon and $s_{1}$ is the ending site of the ribbon. We also assume that ribbons avoid self-crossing.

Definition 3.2.7. For any ribbon $\xi$ with end sites $s_{0}$ and $s_{1}$, and $g, h \in G$ the ribbon operator $F_{\xi}^{h, g}$ is defined as is shown in figure 3.12 . 


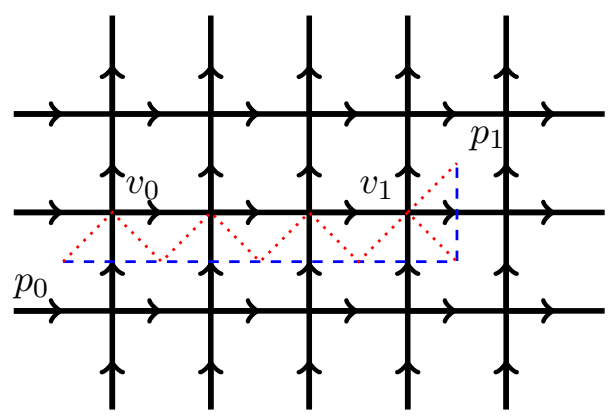

Figure 3.11: A ribbon $\xi$ on an oriented lattice $\mathcal{L}$, from $s_{0}=\left(v_{0}, p_{0}\right)$ to $s_{1}=\left(v_{1}, p_{1}\right)$
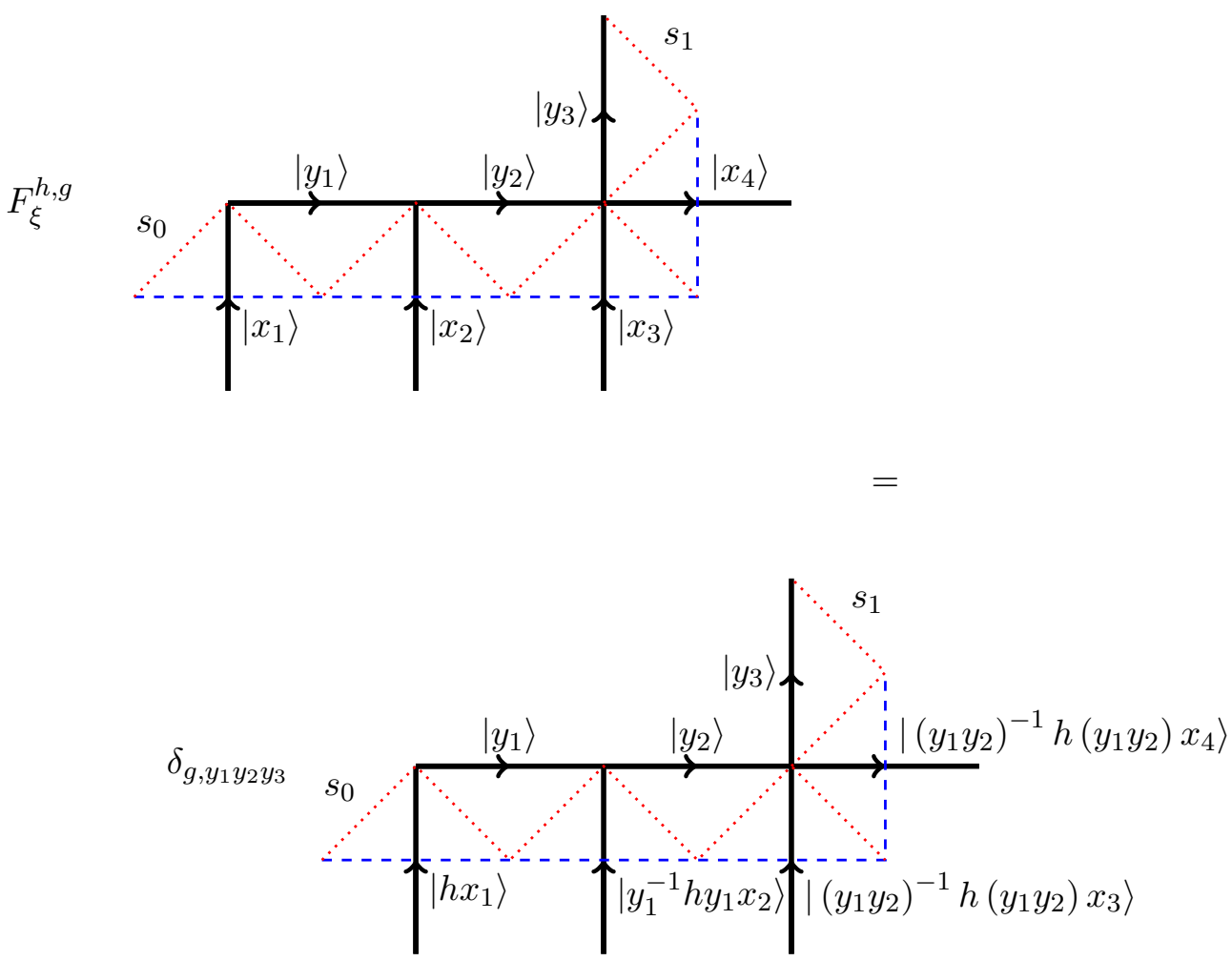

Figure 3.12: Definition of a ribbon operator $F_{\xi}^{h, g} \cdot \xi$ is the ribbon connecting the sites $s_{0}$ and $s_{1}$

By direct calculation, is easy to verify that the ribbon operators satisfy the following commutation relations:

$$
\begin{aligned}
F_{\xi}^{h, g} F_{\xi}^{h^{\prime}, g^{\prime}} & =\delta_{g, g^{\prime}} F_{\xi}^{h h^{\prime}, g}, \\
\left(F_{\xi}^{h, g}\right)^{\dagger} & =F_{\xi}^{h^{-1}, g} .
\end{aligned}
$$


Moreover, for every site $t$ different from $s_{0}$ and $s_{1}$ we also have

$$
\begin{aligned}
{\left[F_{\xi}^{h g}, A_{t}^{k}\right] } & =0, \\
{\left[F_{\xi}^{h g}, B_{t}^{k}\right] } & =0, \\
A_{s_{0}}^{k} F_{\xi}^{h, g} & =F_{\xi}^{k h k^{-1}, k g} A_{s_{0}}^{k}, \\
B_{s_{0}}^{k} F_{\xi}^{h, g} & =F_{\xi}^{h, g} B_{s_{0}}^{k h}, \\
A_{s_{1}}^{k} F_{\xi}^{h, g} & =F_{\xi}^{h, g k^{-1}} A_{s_{1}}^{k}, \\
B_{s_{1}}^{k} F_{\xi}^{h, g} & =F_{\xi}^{h, g} B_{s_{1}}^{g^{-1} h^{-1} g k} .
\end{aligned}
$$

As before, let $\left|\psi_{0}\right\rangle$ be the ground state of the Hamiltonian $H_{Q D}$ fulfilling the conditions 3.28. Let us now consider a ribbon $\xi$ connecting the two sites $s_{0}$ and $s_{1}$. By the previous relations we see that $F_{\xi}^{h, g}$ commutes with all terms of $H_{Q D}$ except for the endpoints of the ribbon sites $s_{0}, s 1$. In other words, the state $\left|\psi^{h, g}\right\rangle=F_{\xi}^{h^{-1}, g}\left|\psi_{0}\right\rangle$ satisfies all constraints of the Hamiltonian except the ones at $s_{0}$ and $s_{1}$. Moreover, as it is stressed on the notation used $\left|\psi^{h, g}\right\rangle$ does not depend on the ribbon $\xi$, but only on the end points $s_{0}$ and $s_{1}$. This means that taking any other ribbon $\xi^{\prime}$ having the same end points, the relation

$$
\left|\psi^{h, g}\right\rangle=F_{\xi}^{h^{-1}, g}\left|\psi_{0}\right\rangle=F_{\xi^{\prime}}^{h^{-1}, g}\left|\psi_{0}\right\rangle
$$

holds. From the previous, applying $F_{\xi}^{h, g}$ on the ground state can be thought of as creating a pair of quasi-particles at sites $s_{0}$ and $s_{1}$.

On the other hand, their braidings can be defined when moving these quasi-particles while extending the corresponding ribbons. In this line of thought, to fuse two quasiparticles of this form is sufficient to move them to the same site. The set of these quasi-particles describes a system of anyons.

Let us introduce the space of quasi-particle excitations living at $s_{0}$ and $s_{1}$ is to be

$$
\mathcal{L}\left(s_{0}, s_{1}\right)=\left\{|v\rangle: A_{t}|v\rangle=B_{t}|v\rangle=|v\rangle \forall t \neq s_{0}, s_{1}\right\}
$$

that has been shown to be spanned by the states $\left|\psi^{h, g}\right\rangle[24]$.

Remark 3.2.8. By the above argument, all excitations in this system can be obtained by applying ribbon operators on the ground state $\left|\psi_{0}\right\rangle$. This is, for the quantum double models, the excitations are completely characterized.

The procedure to find this system of anyons (quasi-particles) is as follows. Let $t$ be a site different from $s_{0}, s_{1}$, and let $\zeta$ be a closed ribbon encircling $s_{0}$ but not $s_{1}$, and both of whose end points are $t$. The unknown excitation at $s_{0}$ can be characterized by creating a particle-antiparticle pair at $t$, move one of them along $\zeta$ and rotate it around $s_{0}$. Over 
$t$, by measuring the vertex and plaquette operators we can check whether the pair (after braiding) fuses to vacuum or not. We can identify the anyon at $s_{0}$ by repeating this process for different particle-antiparticle pairs that we create at $t$. Creating a particleantiparticle pair and moving one of them, correspond to applying some ribbon operator along $\zeta$. Moreover, if the pair fuses to vacuum, this ribbon operator should not create any excitation at $t$, i.e. besides the vertex and plaquette operators along $\zeta$, this ribbon operator must commute with $A_{t}$ and $B_{t}$ as well.

Letting $\mathcal{F}_{\zeta}$ to be the algebra of ribbon operators $F_{\zeta}^{h, g}, g, h \in G$, it has been argued in [69] that the anyon-types are characterized by the subalgebra $\mathcal{K}_{\zeta} \subseteq \mathcal{F}_{\zeta}$ of ribbon operators which commute with $A_{t}$ and $B_{t}$ :

$$
\mathcal{K}=\left\{T \in \mathcal{F}_{\zeta}:\left[T, A_{t}\right]=\left[T, B_{t}\right]=0\right\},
$$

In [25], for any irreducible representation $X$ of $D(G)$ a ribbon operator $T^{X} \in \mathcal{K}_{\zeta}$ is defined, and it is proved that $\mathcal{K}_{\zeta}$ is generated by these operators. In this context the following equations hold:

$$
\begin{aligned}
\left(T^{X}\right)^{\dagger} & =T^{X}, \\
T^{X} T^{Y} & =\delta_{X, Y} T^{X}, \\
\sum_{X} T^{X} & =I,
\end{aligned}
$$

Remark 3.2.9. As a result, anyon-types are in one-to-one correspondencewith irreps of $D(G)$.

\subsection{Examples of generalizations}

This section presents two generalizations of the QDM based on Hopf algebraic methods that broaden the capacity of the Drinfeld Double structure $D(G)$. The names 1-2-gauge and 2-3-gauge were coined having in mind the framework presented in the next Chapters, so its justification is delayed until then. These particular models were developed by our research group [50,51], as an attempt to go beyond the mere lattice gauge picture. It considers further degrees of freedom associated to the vertices of a lattice $\mathcal{L}$.

In a loose sense, Hopf's algebras can be considered as generalizations of groups, this is because of them being nonconmutative algebras of functions on groups. Let us first define the Hopf Algebras:

Definition 3.3.1 (Hopf algebra). A Hopf algebra is the quintuple $(\mathcal{A}, m, \eta, \Delta, \varepsilon, S)$ which is a (associative and co-associative) bi-algebra $\mathcal{A}$, i.e. with product $m$, coproduct 
$\Delta$, integral $\eta$ and cointegral $\epsilon$, over a field $\mathbb{K}$, together with a $\mathbb{K}$-linear map $S: \mathcal{A} \rightarrow \mathcal{A}$ called the antipode such that the diagram shown in figure (3.13) commutes.

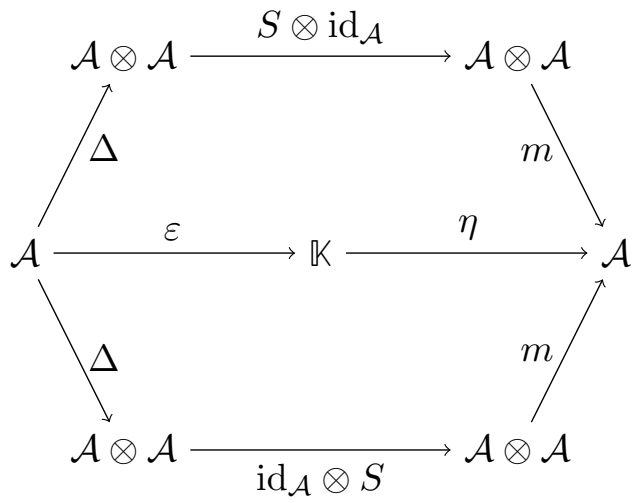

Figure 3.13: The commuting diagram of a Hopf Algebra

It has been shown in [50] that the QDM can be recovered by representing their operators $A_{v}$ and $B_{p}$ as composed by combinations of the maps $m: \mathcal{A} \otimes \mathcal{A} \rightarrow \mathcal{A}$ and $\Delta: \mathcal{A} \rightarrow$ $\mathcal{A} \rightarrow \mathcal{A}$, whose forms is not very illustrative for the present discussion. However, both maps encode the group structure in a natural way. The only foreign element here may be the antipode $S: \mathcal{A} \rightarrow \mathcal{A}$. It can be simply stated that its presence is justified for compatibility reasons between the algebra and the coalgebra structures.

\subsubsection{The "1-2 gauge" theory à la quantum double model}

This case consider degrees of freedom over the links and vertices of a square lattice $\mathcal{L}$. In order to couple the pure gauge with the vertices, we use a Hopf comodule structure:

Definition 3.3.2 (Hopf comodule). Let $\mathcal{A}$ be a Hopf algebra. A right-comodule $\mathcal{M}$ over $\mathcal{A}$ is a $k$-vector space $\mathcal{M}$ and a linear map $\mu: \mathcal{M} \rightarrow \mathcal{M} \otimes \mathcal{A}$ such that the diagrams 3.14 and 3.15 commute. The left-comodule can be defined analogously.

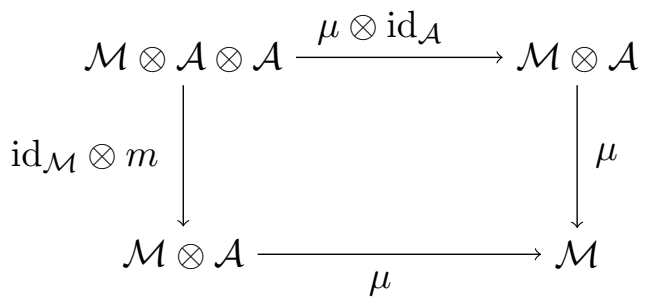

FiguRE 3.14: The right co-module

Using the latter structure, we can find operators that resemble those of the QDM. Again, we are not showing the explicit formalism here since our exposition is informal 


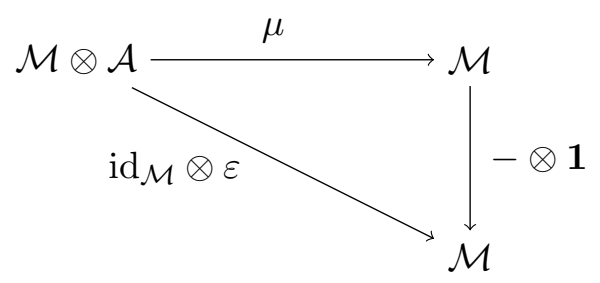

FiguRE 3.15: The right co-module

and motivational. For those interested readers, the details can be checked in [50]. The picture follows similarly to that we have already exposed in the case of pure gauge. These models are built by taking the QDM Hilbert space and expand it to include the vertices of the lattice $\mathcal{L}$ in the following way

$$
\mathcal{H}_{\mathrm{QDMv}}=\mathcal{H}_{\mathrm{QDM}} \otimes\left(\bigotimes_{v \in \mathcal{L}} \mathcal{H}_{v}\right)
$$

where $\mathcal{H}_{v}$ stands for the $n$-dimensional Hilbert space associated to the $v$-th vertex. We take the orthonormal basis of these spaces to be finite

$$
\mathcal{B}_{v}=\left\{|\alpha\rangle \mid \alpha \in G_{0}, \operatorname{span}(|\alpha\rangle)=\mathcal{H}_{v}\right\}
$$

where $G_{0}$ is a group. We consider $\mathcal{H}_{v}=\mathbb{C}\left[G_{1}\right]$ and a $\mathbb{C}\left[G_{1}\right]$-module structure, specified by the map $\mu: \mathcal{H}_{l} \times \mathcal{H}_{v} \rightarrow \mathcal{H}_{v}$

$$
(g, \alpha) \mapsto \beta=\mu(g, \alpha) \quad \forall g \in G_{1}, \forall \alpha \in G_{0} .
$$

Three operators are defined in this setting: a modified vertex operator $\bar{A}_{v}$, a plaquette operator $B_{p}$ and a link operator $C_{l}$ (See Figures 3.16 and 3.17).

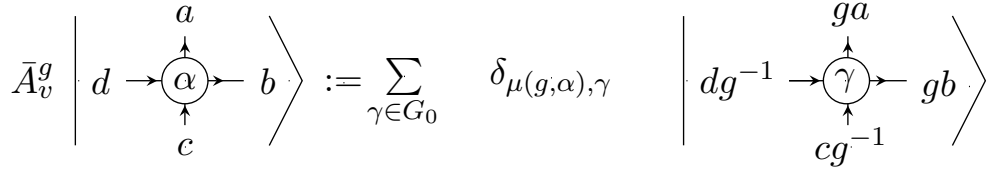

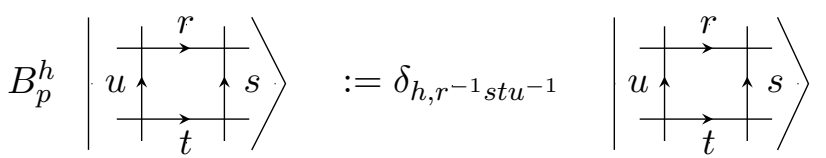

$$
\begin{aligned}
& C_{l}\left|\alpha \rightarrow \vec{v} \beta:=\delta_{\mu(v, \alpha), \beta} \quad \alpha \rightarrow \vec{v} \beta\right\rangle
\end{aligned}
$$

Figure 3.16: $\quad \bar{A}_{v}^{g}, \quad B_{p}^{h}$ and $C_{l}$ defined over local regions of $\mathcal{L}$, for $a, b, c, d, g, h, r, s, t, u, v \in G_{1}$ and $\alpha, \beta, \gamma \in G_{0}$. Notice that $B_{p}^{h}$ is the same plaquette operator as the one defined for the QDM case. 
The dynamics of these models is determined by the frustration free Hamiltonian

$$
H_{1-2}:=-\sum_{v} \bar{A}_{v}-\sum_{p} B_{p}-\sum_{l} C_{l}
$$

where $\bar{A}_{v}:=\frac{1}{\left|G_{1}\right|} \sum_{g \in G_{1}} \bar{A}_{v}^{g}, B_{p}:=B_{s}^{e}$ and $C_{l}$, are constructed to be commuting operators, thus, this Hamiltonian is soluble.

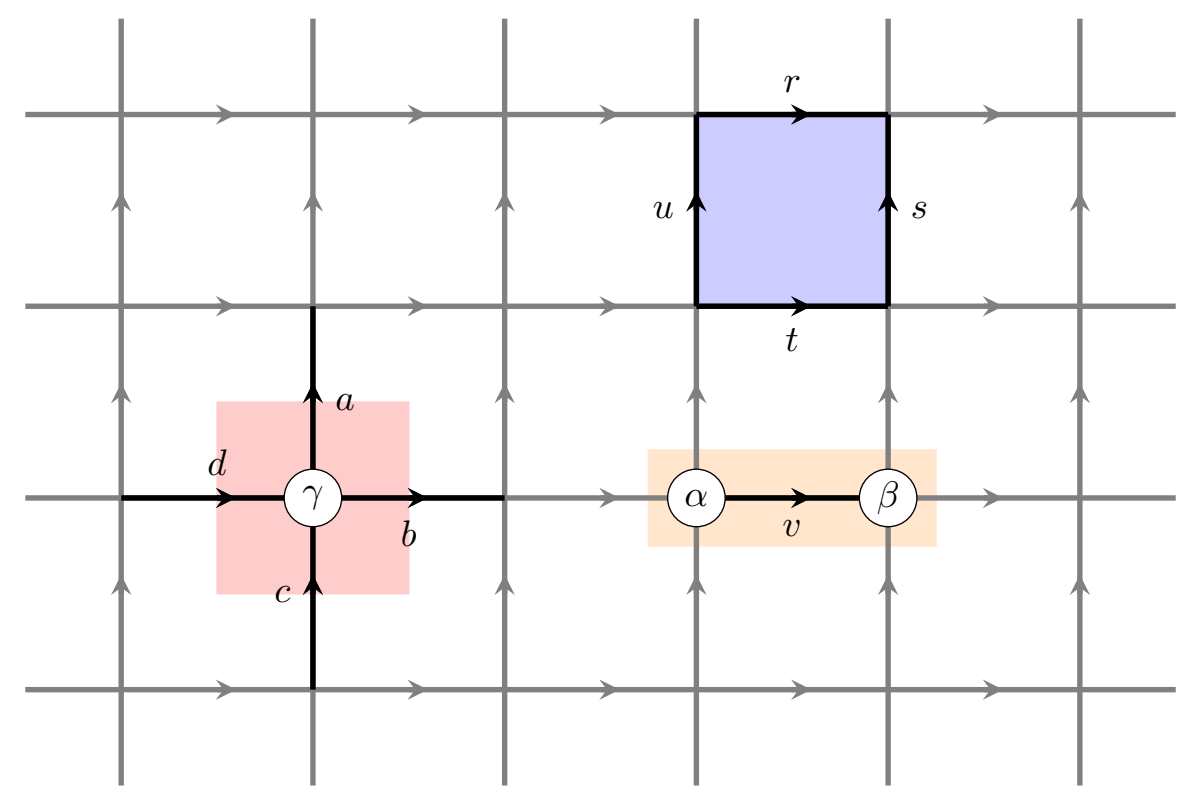

FigURE 3.17: Section of an oriented lattice $\mathcal{L}$ that give support to the QDMv, where (i) the blue and green sectors have already been defined in Figure 4.4 and (ii) the new orange sector is associate to $l$-th shared link by two adjacent vertices containing discrete matter fields.

The commutation requirement of the aforementioned operators, force the action $\mu$ to be a group homomorphism that acts as a permutation of the base $|\alpha\rangle$. In other words, we have the following restrictions for map $\mu$ :

$$
\begin{aligned}
\mu(g, \alpha) & :=\mu_{g} \triangleright \alpha \quad \text { where } \quad \mu_{g} \quad \text { is a permutation operator, } \\
\mu_{1} & =\operatorname{id}_{\mathcal{B}_{v}}, \\
\left(\mu_{g}\right)^{\dagger} & =\mu_{g^{-1}} \quad \text { and } \\
\mu_{g_{1}} \triangleright\left(\mu_{g_{2}} \triangleright \alpha\right) & =\mu_{g_{1} \cdot g_{2}} \triangleright \alpha .
\end{aligned}
$$

Note that, although we are considering a left-module, a right module can be also used.

As it is the case with QDM, the 1-2-gauge spectrum can similarly be obtained by a characterization of a ground state $\left|\Psi_{0}\right\rangle$, satisfying

$$
\bar{A}_{v}\left|\Psi_{0}\right\rangle=\left|\Psi_{0}\right\rangle, B_{p}\left|\Psi_{0}\right\rangle=\left|\Psi_{0}\right\rangle \text { and } C_{l}\left|\Psi_{0}\right\rangle=\left|\Psi_{0}\right\rangle
$$


It can be easily checked that the vacuum subspace of this model is invariant under the projector operator

$$
P_{1-2}=\prod_{v} \bar{A}_{v} \prod_{p} B_{p} \prod_{l} C_{l}
$$

However,

Remark 3.3.3. The study of the excited states have not been systematized yet. No analog of the ribbon operators have been found. In fact, the evidence suggests that for these models a case by case study must be done.

\subsubsection{The "2-3 gauge" theory à la quantum double model}

This is also a development of the group that, however, has not been yet published. And some of the details were treated in the Ph.D. thesis of Maria Fernanda de Resende (yet to be puclished). Without loss of generality, let us consider the dual lattice $\mathcal{L}^{*}$ (See Figure 3.18), we note that the plaquette centroids of $\mathcal{L}$ can be interpreted as vertices of a dual lattice $\mathcal{L}^{*}$. Indeed, this rationale can be generalized to show that it is possible to construct another model with degrees of freedom over the plaquette by algebraically dualizing the 1-2-gauge model.

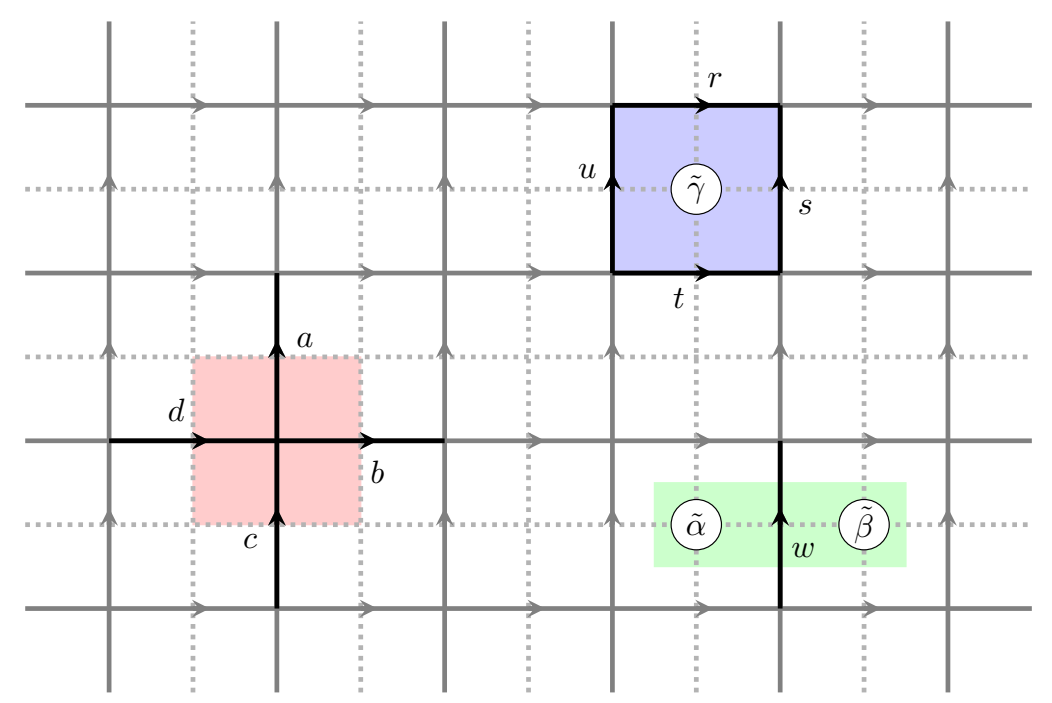

Figure 3.18: Oriented lattice $\mathcal{L}$ (continuous) and its dual lattice $\mathcal{L}^{*}$ (dotted).

We start in an analogous fashion as it was done for the QDMv case, let us extend the QDM Hilbert space as

$$
\mathcal{H}_{\mathrm{QDMp}}=\left(\bigotimes_{p \in \mathcal{L}} \mathcal{H}_{p}\right) \otimes \mathcal{H}_{\mathrm{QDM}}
$$


where, now $\mathcal{H}_{p}$ represent an $m$-dimensional Hilbert space associated to $p$-th vertex of $\mathcal{L}^{*}$. We write the orthonormal basis

$$
\mathcal{B}_{p}=\left\{|\tilde{\alpha}\rangle \mid \tilde{\alpha} \in G_{2}, \operatorname{span}(|\tilde{\alpha}\rangle)=\mathcal{H}_{p}\right\}
$$

where $G_{2}$ is a monoid. In analogy to the QDMv case, we take $\mathcal{H}_{p}=\mathbb{C}\left(G_{2}\right)$ we use a module structure $\alpha: \mathcal{H}_{p} \rightarrow \mathcal{H}_{p} \times \mathcal{H}_{l}$ :

$$
|\tilde{\alpha}\rangle \mapsto|\tilde{\alpha}\rangle \otimes|f(\tilde{\alpha})\rangle, \quad \text { with } \quad f: G_{2} \rightarrow G_{1}
$$

such that

$$
\begin{aligned}
f(1) & =1, \\
(f(\tilde{\alpha}))^{\dagger} & =f\left(\tilde{\alpha}^{-1}\right)=f^{-1}(\tilde{\alpha}) \text { and } \\
f\left(\tilde{\alpha}_{1}\right) \cdot f\left(\tilde{\alpha}_{2}\right) & =f\left(\tilde{\alpha}_{1} * \tilde{\alpha}_{2}\right)
\end{aligned}
$$

being $\tilde{\alpha}^{-1}$ the inverse element of $\tilde{\alpha}$. Moreover, by compatibility reasons of the mathematical structures, we must impose $f(\tilde{\alpha}) \in \mathcal{Z}\left(G_{1}\right)$, the center of the group $G_{1}$, a condition that forces the product $*$ to be symmetric, and consequently, $G_{2}$ to be Abelian.

This new dualized picture leads to vertex following operators: a vertex operator $A_{v}^{g}$ exactly as in the QDM, a modified plaquette operator $\bar{B}_{s}^{h}$ and a new dual link operator $D_{l}^{\tilde{\gamma}}$, defined in Figure 3.19, allowing for the definition of the frustration free Hamiltonian

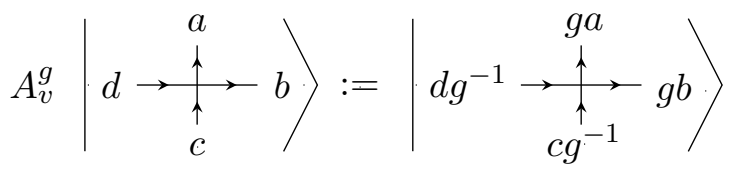

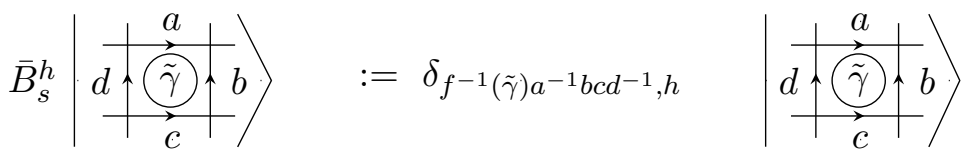

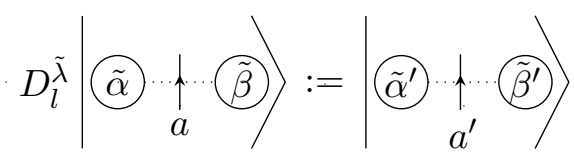

Figure 3.19: Definition of the $A_{v}^{g}, \bar{B}_{p}^{h}$ and $D_{l}^{\tilde{\lambda}}$. Where $a, b, c, d, g \in G_{1}$ and $\tilde{\alpha}, \tilde{\beta}, \tilde{\gamma} \in G_{2}$ with $a^{\prime}=f(\tilde{\lambda}) \cdot a, \tilde{\alpha}^{\prime}=\tilde{\alpha} * \tilde{\lambda}$ and $\tilde{\beta}^{\prime}=\tilde{\lambda}^{-1} * \tilde{\beta}$.

$$
H_{2-3}:=-\sum_{v} A_{v}-\sum_{p} \bar{B}_{p}-\sum_{l} D_{l}
$$

where $A_{v}:=\frac{1}{\left|G_{1}\right|} \sum_{g \in G_{1}} \bar{A}_{v}^{g}, \bar{B}_{p}:=\bar{B}_{s}^{e}$ and $D_{l}:=\frac{1}{\left|\tilde{G}_{2}\right|} \sum_{\tilde{\lambda}_{\epsilon} \tilde{G}_{2}} D_{l}^{\tilde{\lambda}}$ are commuting operators. The latter turns $\mathrm{H}_{2-3}$ into a soluble one. 
Moreover, as before, we note that the ground state $|\tilde{\Psi}\rangle$ can be obtained by the characterization:

$$
A_{v}\left|\tilde{\Psi}_{0}\right\rangle=\left|\tilde{\Psi}_{0}\right\rangle, \bar{B}_{p}\left|\tilde{\Psi}_{0}\right\rangle=\left|\tilde{\Psi}_{0}\right\rangle \text { and } D_{l}\left|\tilde{\Psi}_{0}\right\rangle=\left|\tilde{\Psi}_{0}\right\rangle
$$

Once again, the vacuum subspace is invariant under the action of the projector

$$
P_{1-2}=\prod_{v} A_{v} \prod_{p} \bar{B}_{p} \prod_{l} D_{l}
$$

The same as in the precious model:

Remark 3.3.4. The study of the excited states have not been systematized yet. No analog of the ribbon operators have been found. In fact, the evidence suggests that for these models a case by case study must be done.

\subsection{Comments}

This chapter have tried to introduce the main features lattice gauge theories, as well as the properties of the 2-dimensional quantum double models. In fact, we can recognize all the characteristics of the lattice gauge as being present in the QDM formalism. This justifies the claim that, from a quantum fields perspective, these models are effectively gauge theories over the lattice. Is is clear that the classic two dimensional case presented here is well understood and, even further, the formalism of the ribbon operators is suitable to study and classify its interactions. This well roundedness can be partially explained by the high symmetry of the two dimensional case.

This is, of course, not the case when generalizations with more degrees of freedom or higher dimensional situations are considered. This claim was exemplified by the 1-2gauge theory (a two dimensional QDM coupled with degrees of freedom at the vertices). This model mostly conserves the characteristics of the QDM at the level of the ground state but the topological ones, which are not straight forward to prove anymore due to lack of clear geometrical interpretation. In fact, this compromise of geometrical interpretation will be repeated in the general formalism. Also, although it was not discussed, the study of these models show that that the ribbon operators have not clear analogs when generalizations are considered, either in higher dimensions or further degrees of freedom. It is evident that there is a need for more structure in order to understand these models. We address many of these problems, as well as explain many of the features of these models, in the next chapter. In can also be mentioned that we 
worked on a very similar construction that is based in a Hopf module instead of a comodule. With the difference that the degrees of freedom are associated to the dual links and will result in an analog operator $D_{l}$, this time resembling a modified $A_{v}$ operator that is coupled with this new structure.

Both examples hint at an underlying general structure behind these generalizations. In each case there are new operators that couple the 1-gauge with the new degrees of freedom. Furthermore, keeping the non-abelian structure of the 1-gauge summed up by the group $G_{1}$ in the QDM case, imposes somewhat severe restrictions on the type of groups $G_{0}$ and $G_{2}$ that can be coupled without breaking the features of the first. This is a reason to compromise the non abelian-ness to generalize in higher dimensions, which we will see over the next chapter. 


\section{Chapter 4}

\section{Higher dimensional abelian gauge theories}

We have stated so far that the finite case of 1-gauge topological theories, when no global symmetries are present, is fairly well understood and classified. In recent years, several attempts to generalize the latter through the so called 2-gauge theories have opened the door to interesting new models and new topological phases, not described by the previous schemes of classification. However, this program becomes cumbersome when tackling the 3 -gauge situation, since several restrictions are imposed into the mathematical structure in way of the makes the theory almost trivial, or at least intractable.

We take a different path, we go even beyond the 2-gauge construction by considering a class of models that live in arbitrary higher dimensions, although we compromise in structure by considering only abelian gauge. These models are constructed by means of chain complexes of abelian groups, forcing to generalized the usual notion of gauge configurations into a more general concept. The advantage of such an approach is that, the topological order is obtained explicitly and a closed expression is shown, when the ground state space of these models is described.

We change the tone into a formal one in order to rigorously prove all the relevant points presented throughout the discussion. The following construction makes heavy use of cohomology with coefficients in a finite chain complex described by Brown [52] and others. We have included all the relevant mathematical framework in two separate Appendices $\mathrm{B}$ and $\mathrm{C}$ to not to interrupt the easy reading of the chapter.

We emphasize that the clear advantage of using the formalism presented below is that we are able to encompass into a single, fairly comprehensive and very unified fashion, a broad class of models already available in the literature, namely the QDM and abelian 
generalization. In the same token, two of such models are exemplified by the end of this section.

\subsection{Hilbert Space and Quantum States}

Let us start with some basic technical details. Let $\mathbb{C}[G]$ be the group algebra of a discrete group $G$. We make use of the canonical way in which a Hilbert space can be constructed by means of defining a $C^{*}$ structure. The overall procedure is sketched as follows: First, let $A$ be the $C^{*}$-algebra $C^{*}(G)$, defined to be the $C^{*}$-enveloping algebra of $L^{1}(G)$, i.e:

$$
\|f\|_{A}:=\sup _{\pi}\|\pi(f)\|,
$$

where $\pi$ is a non-degenerate bounded $*$-representation that ranges over all non-degenerate *-representations of group algebras of topological groups $C_{c}(G)$ on Hilbert spaces. Moreover, since $G$ is discrete, the well-definiteness of the previous norm is then ensured by the triangular inequality. Consequently, any $*$-homomorphism from $\mathbb{C}[G]$ to some $C^{*}$ algebra of bounded operators on some Hilbert space, factors through the inclusion map $\mathbb{C}[G] \hookrightarrow C_{\max }^{*}(G)$.

Also, we recall that, for a field $k$, the spaces $k\left[G_{1}\right] \otimes_{k} k\left[G_{2}\right] \simeq k\left[G_{1} \times G_{2}\right]$, which can be generalized inductively for finite products. Under these circumstances, it is clear that the following construction is well defined:

Definition 4.1.1 (Hilbert Space and States). We define the Hilbert space $\mathcal{H}$, with its corresponding inner product $\langle\cdot, \cdot\rangle:=\|\cdot\|_{2}^{2}$ as

$$
\mathcal{H}:=\bigotimes_{n} \bigotimes_{x \in K_{n}} \mathbb{C}\left[G_{n}\right], \quad \text { and states } \quad|f\rangle:=\bigotimes_{n} \bigotimes_{x \in K_{n}}\left|f_{n}(x)\right\rangle \forall f \in \operatorname{hom}(C, G)_{0}
$$

where $\left\{K_{n}\right\}$ is a free abelian finitely generated set of generators $K_{n},\left\{G_{n}\right\}$ is a graded group, $\mathbb{C}\left[G_{n}\right]$ is the group algebra of the group $G_{n}$ and $\operatorname{hom}(C, G)_{0}$.

The set hom $(C, G)_{0}$ has been discussed in Definition C.2.1, but that we recall it here for convenience. For all $p \in \mathbb{Z}$, let $\operatorname{hom}(C, G)_{0}$ be the following abelian group

$$
\operatorname{hom}(C, G)_{0}:=\prod_{n} \operatorname{Hom}\left(C_{n}, G_{n}\right)
$$

where $\operatorname{Hom}\left(C_{n}, G_{n}\right)$ denotes the abelian group formed by morphisms between $C_{n}$ and $G_{n}$ In FIG. 4.1) we show the situation, where the maps $f_{n}$ involved in the previous 
definition are shown to connect each $C_{n}$ of the upper chain with its corresponding $G_{n}$ of the lower chain.

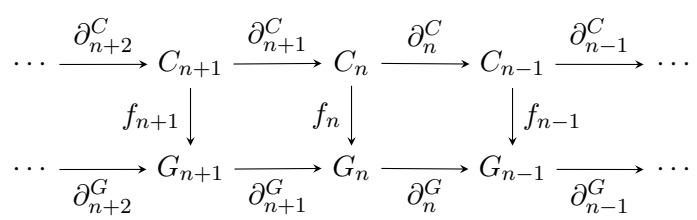

Figure 4.1: Diagrammatic description for $f \in \operatorname{hom}(C, G)_{0}$.

Indeed, when denoting the finite product of cartesian products by $\Pi_{n}$ the above discussion implies that

$$
\bigotimes_{n} \bigotimes_{x \in K_{n}} \mathbb{C}\left[G_{n}\right]=\mathbb{C}\left[\prod_{n} G_{n}^{\left|K_{n}\right|}\right]=\mathbb{C}[\Gamma], \quad \text { with } \quad \Gamma:=\prod_{n} G_{n}^{\left|K_{n}\right|}
$$

a finite abelian group, which shows the well definiteness of the Hilbert space $\mathcal{H}$ claimed above. On the other hand, since each $G_{n}$ has been taken to be a finite group, the Hilbert space $\mathcal{H}$ will be finite when $\left\{K_{n}\right\}$ is finite. This, in practice, will not be a problem, since the applications, particularly the ones treated here take $\left\{K_{n}\right\}$ as being the generators of a finite dimensional simplicial complex.

As it is clearly expressed in the Definition 4.1.1, the novel feature of this construction is that the basis states are now related to elements of the set hom $(C, G)_{0}$. As such, each element $x \in K_{n}$ has a single state $\left|f_{n}(x)\right\rangle \in \mathbb{C}\left[G_{n}\right]$ associated to it, indexed by a group element $f_{n}(x) \in G_{n}$. In other words, there is an injection between what we can call a state configuration and the morphisms $f \in \operatorname{hom}(C, G)_{0}$.

We notice that, thus stated, this construction obscures the clear geometrical interpretation of the model but, we will see that the formalism will pay off when tackling the problem of analyzing the ground state degeneracy of the model later on.

\subsubsection{Operators and Algebraic Relations}

We now define the quantum operators acting over the Hilbert space $\mathcal{H}$. It is not a surprise that these operators resemble the ones described in Chapter ?? for the QDM case. In fact, they will be to be equivalent in the in their abelian version.

Definition 4.1.2 (Shift and Clock operators). Let $|f\rangle \in \mathcal{H}$ with $f \in \operatorname{hom}(C, G)_{0}$. Given $t \in \operatorname{hom}(C, G)_{0}$ and $m \in \widehat{\operatorname{hom}}(C, G)_{0}$, we define the operators

$$
P_{t}|f\rangle:=|f+t\rangle, \quad Q_{m}|f\rangle:=\chi_{m}(f)|f\rangle
$$


called the shift and the clock operators, respectively.

From the above definition is immediate that:

Lemma 4.1.3 (Shift and Clock operators algebra). Let $t, t_{1}$ and $t_{2} \in \operatorname{hom}(C, G)_{0}$ and $m, m_{1}$ and $m_{2} \in \widehat{h o m}(C, G)_{0}$, the following relations are satisfied.

$$
P_{t_{1}} P_{t_{2}}=P_{t_{1}+t_{2}}, \quad Q_{m_{1}} Q_{m_{2}}=Q_{m_{1}+m_{2}}, \quad P_{t} Q_{m}=\chi_{m}(t) Q_{m} P_{t}
$$

Proof. Straightforward when applying the operators of Definition 4.1.2 to a general basis state as in Definition 4.1.1.

As obvious as it might seem, the previous definitions allows us to study any of the quantum systems presented from an operator perspective only, which will prove to be of advantage along the following sections. This is nothing more than the analog of the well known equivalence between the Schrödinger and the Heisenberg picture of quantum mechanics. This observation will turn out to be of great help when studying the ground state subspace. More precisely, we have:

Remark 4.1.4 (Equivalence between states and shift operators). The trivial map $0 \in$ $\operatorname{hom}(C, G)_{0}$ allows us to write any $|f\rangle \in \mathcal{H}$ in the form $|f\rangle=P_{f}|0\rangle$.

Before continuing, we recall the following canonical constructions for dual spaces. For general references we derive the reader to $[72,73]$ :

Let $H$ be a finite abelian group. Any irreducible representation $\rho$ of $\mathrm{H}$ is one-dimensional and is completely determined by its character $\chi_{\rho}: H \rightarrow U(1)$. Let $\hat{H}$ be the set of irreducible representation, the tensor product defines an associative binary operation $\left(\rho, \rho^{\prime}\right) \in \hat{H} \times \hat{H} \rightarrow \rho \otimes \rho^{\prime} \in \hat{H}$ which we denote additively for convenience, so $\rho+\rho^{\prime}:=$ $\rho \otimes \rho^{\prime}$. Moreover, it is well known that $\hat{H}$ is actually a finite abelian group under the tensor product and that it is isomorphic to the multiplicative group formed by $\left\{\chi_{\rho} \mid \rho \in \hat{H}\right\}$ due to the fact that:

$$
\chi_{\rho+\rho^{\prime}}=\chi_{\rho \otimes \rho^{\prime}}=\chi_{\rho} \chi_{\rho^{\prime}}
$$

We also recall that, given a morphism $f: H \rightarrow H^{\prime}$ between finite abelian groups, there is a dual morphism $\hat{f}: \hat{H}^{\prime} \rightarrow \hat{H}$ defined by $\rho \mapsto \hat{f}(\rho)=\rho \circ f$.

Proposition 4.1.5. The morphism $\hat{f}$ is a group morphism and $\chi_{\hat{f}(\rho)}=\chi_{\rho} \circ f$. 
Proof. The following holds,

$$
\begin{aligned}
\chi_{\hat{f}(\rho)}(h) & =\operatorname{Tr}(\hat{f}(\rho)(h)) \\
& =\operatorname{Tr}(\rho(f(h))) \\
& =\chi_{\rho}(f(h))
\end{aligned}
$$

or $\chi_{\hat{f}(\rho)}=\chi_{\rho} \circ f$ as claimed. It follows also that:

$$
\begin{aligned}
\chi_{\hat{f}\left(\rho+\rho^{\prime}\right)} & =\chi_{\rho+\rho^{\prime}} \circ f \\
& =\chi_{\rho} \chi_{\rho^{\prime}} \circ f \\
& =\left(\chi_{\rho} \circ f\right)\left(\chi_{\rho^{\prime}} \circ f\right) \\
& =\chi_{\hat{f}(\rho)} \chi_{\hat{f}\left(\rho^{\prime}\right)} \\
& =\chi_{\hat{f}(\rho)+\hat{f}\left(\rho^{\prime}\right)}
\end{aligned}
$$

and $\chi_{\hat{f}(0)}=\chi_{0} \circ f=1=\chi_{0}$ which implies $\hat{f}\left(\rho+\rho^{\prime}\right)=\hat{f}(\rho)+\hat{f}\left(\rho^{\prime}\right)$ and $\hat{f}(0)=0$.

Hence, we can introduce the cochain complex hom $(C, G)=\left(\operatorname{hom}(C, G)_{p}, \delta\right)$ :

$$
\ldots \rightarrow \operatorname{hom}(C, G)_{p} \stackrel{\delta_{p}}{\rightarrow} \operatorname{hom}(C, G)_{p+1} \rightarrow \ldots
$$

where $\delta_{p}$ is given by $\left(\delta_{p} f\right)_{n}:=f_{n-1} \partial_{n}^{C}-(-1)^{p} \partial_{n-p}^{G} f_{n}$, and its dual, the chain complex $\widehat{\operatorname{hom}}(C, G):=\left(\widehat{\operatorname{hom}}(C, G)_{p}, \widehat{\delta}\right)$ :

$$
\cdots \leftarrow \widehat{\operatorname{hom}}(C, G)_{p} \stackrel{\hat{\delta}_{p+1}}{\longleftarrow} \widehat{\operatorname{hom}}(C, G)_{p+1} \leftarrow \ldots
$$

Given the latter, we can introduce two sets of operators mostly comprised of combinations of the simple shift and clock operators of Definition 4.1.2. These operators will compose those eventually appearing in the Hamiltonian of the model. We will also see that they will be relevant not just for the dynamics but for measurement procedures as well. Recalling Definition C.2.1, we have:

Definition 4.1.6 (Generalized gauge transformations and holonomies). For all $t \in$ $\operatorname{hom}(C, G)_{-1}$ and $|f\rangle \in \mathcal{H}$, we define the operator $A_{t}$ as:

$$
A_{t}|f\rangle:=\left|f+\delta_{-1} t\right\rangle, \quad \text { or, equivalently } \quad A_{t}:=P_{\delta_{-1} t} .
$$

Analogously, for all $m \in \widehat{\operatorname{hom}}(C, G)_{1}$ and $|f\rangle \in \mathcal{H}$, we define the operator $B_{m}$ as:

$$
B_{m}|f\rangle:=\chi_{\hat{\delta}_{1} m}(f)|f\rangle, \quad \text { or, equivalently } \quad B_{m}:=Q_{\hat{\delta}_{1} m} .
$$


where the second equalities come directly from Remark 4.1.4.

The following lemma exemplifies the power of the hermetic formalism and notation introduced so far. It easily follows from the previous definition of the generalized gauge and transformations and holonomies:

Lemma 4.1.7 ( $A_{t}$ and $B_{m}$ algebra). Let $A_{t}$ and $B_{m}$ be as defined in 4.1.6. They satisfy the following relations:

$$
A_{t} A_{t^{\prime}}=A_{t+t^{\prime}}=A_{t^{\prime}} A_{t}, \quad B_{m} B_{m^{\prime}}=B_{m+m^{\prime}}=B_{m^{\prime}} B_{m}, \quad A_{t} B_{m}=B_{m} A_{t} .
$$

Proof. The first two relations are obvious from Lemma 4.1.3. The third one reads

$$
\begin{aligned}
A_{t} B_{m} & =\chi_{\hat{\delta}_{1}(m)}\left(\delta_{-1}(t)\right) B_{m} A_{t} \\
& =\chi_{m}\left(\delta_{0} \circ \delta_{-1}(t)\right) B_{m} A_{t} \\
& =B_{m} A_{t}
\end{aligned}
$$

which follows from Lemma C.2.4 and the definitions of the dual morphism of Proposition 4.1.5, as expected.

In other words, we have basically constructed a pair of operators that commute everywhere for every possible combination of parameters. Rather than coming from a strictly geometrical or group restrictions, this property is coming from the characteristics of the chain complex hom $(C, G)$ itself (or equivalently its dual hom(C, $G)$ ).

In order to recover the geometrical intuition, we can think of the chain $C$ as being a simplicial complex. Notice that, up to now, the operators 4.1.7 have no connection with the local structure of $C$. We try to make the link by using the following maps:

Definition 4.1.8 (Localized maps). Let $x \in K_{n}, y \in K_{m}, g \in G_{n+1}, r \in \hat{G}_{n-1}$ and $f \in \operatorname{hom}(C, G)_{0}$. We define $g x^{*} \in \operatorname{hom}(C, G)_{-1}$ and $r x_{*} \in \widehat{\operatorname{hom}}(C, G)_{1}$ as being:

$$
\left(g x^{*}\right)(y):=\left\{\begin{array}{l}
g \text { if } x=y \\
0 \text { otherwise }
\end{array} \quad, \quad \text { and } \quad\left(r x_{*}\right)(f):=r\left(f_{n}(x)\right)\right.
$$

Indeed, as the name implies, the previous maps are localized around $x \in K_{n}$ so they can be used to define local operators. This is clear by noticing: 
(i) We notice that the group $\operatorname{Hom}\left(C_{n}, G_{n-p}\right)$ is equal to $G_{n-p}^{K_{n}}=\left\{f: K_{n} \rightarrow G_{n-p}\right\}$ since $K_{n}$ is a basis for $C_{n}$. Moreover, for $x, y \in K_{n}$ and $g \in G_{n-p}$ one can define

$$
\begin{aligned}
& g x^{*}: K_{n} \rightarrow G_{n-p} \\
& \left(g x^{*}\right)(y)=\left\{\begin{array}{l}
g \text { if } x=y \\
0 \text { otherwise }
\end{array}\right.
\end{aligned}
$$

(ii) Furthermore, these functions provide a basis for $G_{n-p}^{K_{n}}$ since any $f \in G_{n-p}^{K_{n}}$ can be written as $f=\sum_{x} f(x) x^{*}$. The above discussion implies that

$$
\operatorname{hom}(C, G)_{p}=\prod_{n} \operatorname{Hom}\left(C_{n}, G_{n-p}\right)=\prod_{n} G_{n-p}^{K_{n}},
$$

allowing to construct a basis for $\operatorname{hom}(C, G)_{p}$ using the basis for $G_{n-p}^{K_{n}}$. To achieve this we extend $g x^{*} \in G_{n-p}^{K_{n}}$ to $g x^{*} \in \operatorname{hom}(C, G)_{p}$ in the following way

$$
\left(g x^{*}\right)_{m}=\left\{\begin{array}{l}
g x^{*}, \text { if } m=n \\
0, \text { if } m \neq n
\end{array}\right.
$$

and observe that any $t \in \operatorname{hom}(C, G)_{p}$ can be written as

$$
t=\sum_{n} \sum_{x \in K_{n}} t_{n}(x) x^{*}
$$

so these maps provide a basis for $\operatorname{hom}(C, G)_{p}$.

(iii) In a similar fashion, using the canonical dualization procedure, it is immediate from equation (4.1) that

$$
\widehat{\operatorname{hom}}(C, G)_{p}=\prod_{n} \hat{G}_{n-p}^{K_{n}}=\prod_{n} \operatorname{Hom}\left(C, \hat{G}_{n-p}\right)=\operatorname{hom}(C, \hat{G})_{p},
$$

where the last equality comes from Definition C.2.1. By the same token, given $r \in \hat{G}_{n-p}$ and $x \in K_{n}$ we obtain $r x_{*} \in \widehat{\operatorname{hom}}(C, G)_{p}$ defined by

$$
r x_{*}(f)=r\left(f_{n}(x)\right)
$$

and notice that any $s \in \widehat{\operatorname{hom}}(C, G)_{p}$ can be written as

$$
s=\sum_{n} \sum_{x \in K_{n}} s_{n}(x) x_{*}
$$

where $s_{n}(x) \in \hat{G}_{n-p}$ is defined by $s_{n}(x)(g)=s\left(g x^{*}\right)$. 
As a consequence, we have the following proposition:

Proposition 4.1.9. For any generalized operators $A_{t}$ and $B_{s}$, as defined in 4.1.6, the following decompositions holds:

$$
A_{t}=\prod_{n} \prod_{x \in K_{n}} A_{t_{n}(x) x^{*}}, \quad B_{s}=\prod_{n} \prod_{x \in K_{n}} B_{s_{n}(x) x_{*}}
$$

Proof. From the discussion above, it is evident that the first two expressions are obtained straightforwardly by using equations (4.2) and (4.4), respectively.

Therefore, by using the local maps of Definition 4.1.8, we are now in position to define the following local operators:

Definition 4.1.10 (Local projectors). Given $x \in K_{n}, h \in G_{n-1}$ and $s \in \hat{G}_{n+1}$, we define

$$
A_{x}^{s}:=\frac{1}{\left|G_{n+1}\right|} \sum_{g \in G_{n+1}} \chi_{s}(g) A_{g x^{*}}, \quad B_{x}^{h}:=\frac{1}{\left|G_{n-1}\right|} \sum_{r \in \hat{G}_{n-1}} \chi_{r}(h) B_{r x_{*}},
$$

called the local gauge projector and local holonomy projector, respectively. Where $g x^{*}$ and $r x_{*}$ as in Definition 4.1.8.

To make the connection with known models clearer, note that the operator $A_{g x^{*}}$ in the above definition is the one that performs local gauge transformations with parameter $g \in G_{n+1}$ around $x \in K_{n}$. On the other hand, the operator $B_{x}^{h}$ compares the value of the $n$-th holonomy along the boundary of $x \in K_{n}$ with the gauge parameter $h \in G_{n-1}$. Moreover, each of the operators of Definition 4.1.10 actually forms a set of mutually commuting projectors. We formalize the previous comment in the following lemma:

Lemma 4.1.11. For all $x \in K_{n}, y \in K_{m}, s \in \hat{G}_{n+1}, r \in \hat{G}_{m+1}$ and $h \in G_{n-1}, u \in$ $G_{m-1}$, the following relations for the local gauge transformation and local gauge holonomy (Definition 4.1.10) are satisfied:

(i) Pairwise commutation $A_{x}^{s} A_{y}^{r}=A_{y}^{r} A_{x}^{s} ; \quad B_{x}^{h} B_{y}^{u}=B_{y}^{u} B_{x}^{h} ; \quad A_{x}^{s} B_{y}^{u}=B_{y}^{u} A_{x}^{s}$.

(ii) $A_{x}^{s} A_{x}^{r}=\delta(s, r) A_{x}^{s}, \quad B_{x}^{h} B_{x}^{u}=\delta(h, u) B_{x}^{h}$. Where $\delta(h, u)$ is the Kronecker delta

(iii) $\sum_{s \in G_{n+1}^{*}} A_{x}^{s}=i d, \quad \sum_{h \in G_{n-1}} B_{x}^{h}=i d$. Where id is the identity operator.

Proof. We check the three points of Definition 4.1.10 as follows:

(i) This set of relations are immediately satisfied by the pairwise commutation shown in Proposition 4.1.7. 
(ii) It is straightforward to compute

$$
\begin{aligned}
A_{x}^{s} A_{x}^{r} & =\frac{\sum_{b} \sum_{c}}{\left|G_{n+1}\right|^{2}} \chi_{s}(b) \chi_{r}(c) A_{b x^{*}} A_{c x^{*}} \\
& =\frac{\sum_{b} \sum_{c}}{\left|G_{n+1}\right|^{2}} \chi_{r-s}(c) \chi_{s}(b+c) A_{(b+c) x^{*}} \\
& =\frac{1}{\left|G_{n+1}\right|} \sum_{c} \chi_{r-s}(c) A_{x}^{s},
\end{aligned}
$$

from which the result follows, when using the orthonormal relations of the characters. The second expression is obtained analogously.

(iii) We readily compute

$$
\begin{aligned}
\sum_{s \in G_{n+1}^{*}} A_{x}^{s} & =\frac{1}{\left|G_{n+1}\right|} \sum_{g \in G_{n+1}} \sum_{s \in G_{n+1}^{*}} \chi_{s}(g) A_{g x^{*}} \\
& =\sum_{g \in G_{n+1}} \delta(g, 0) A_{g x^{*}} \\
& =A_{0 x^{*}}
\end{aligned}
$$

from which the result follows, also when using the orthonormal relations of the characters. The second expression is obtained analogously.

As mentioned above, ultimately, since the models available in the literature are mostly expressed in terms of local operators, the ones of Definition 4.1.10 are usual the direct link in relating the formalism presented here with those available in the literature, specifically with the QDM and generalizations of Chapter 3. This will become clear by the end of the chapter when we consider two well known examples.

\subsection{Dynamics}

Since the operators described above are all commuting and locally well behaved, the dynamics of the model is then completely determined when defining a Hamiltonian operator depending on them, which we do below. We proceed heuristically at the beginning. Let us consider the index set $\mathcal{I}$ that runs over all the $x \in K_{n}$, for all $n$. We 
then use the following concise notation in terms of vectors:

$$
\begin{array}{ll}
\alpha:=\left(\left\{\alpha_{x}\right\}_{x \in \mathcal{I}}\right)^{\dagger}, & \mathbf{A}^{0}:=\left(\left\{A_{x}^{0}\right\}_{x \in \mathcal{I}}\right)^{\dagger}, \\
\beta:=\left(\left\{\beta_{y}\right\}_{y \in \mathcal{I}}\right)^{\dagger}, & \mathbf{B}^{0}:=\left(\left\{B_{y}^{0}\right\}_{y \in \mathcal{I}}\right)^{\dagger},
\end{array}
$$

where $(\cdot)^{\dagger}$ is the Hermitian conjugate operation and $\alpha_{x}, \beta_{y} \in \mathbb{C}, \forall x, y \in \mathcal{I}$. We define the Hamiltonian $H_{\alpha, \beta}: \mathbb{C}^{2|\mathcal{I}|} \times \mathcal{H} \rightarrow \mathbb{C}^{2|\mathcal{I}|} \times \mathcal{H}$ as the quadratic form:

$$
H_{\alpha, \beta}:=-\operatorname{tr}\left\{\left(\mathbf{A}^{0} \alpha^{\dagger}+\mathbf{B}^{0} \beta^{\dagger}\right) \cdot\left(\mathbf{A}^{0} \alpha^{\dagger}+\mathbf{B}^{0} \beta^{\dagger}\right)^{\dagger}\right\}
$$

where $\operatorname{tr}$ is the trace operator over the elements of the set $\mathcal{I}$. Notice that $H_{\alpha, \beta}$ is manifestly hermitian. If we write the inner product in the set $\mathcal{I}$ as $(\alpha, \beta)_{\mathcal{I}}:=\alpha^{\dagger} \beta$, we can cast the Hamiltonian $H_{\alpha, \beta}$ in the equivalent form:

$$
\begin{aligned}
H_{\alpha, \beta} & =-\|\alpha\|_{\mathcal{I}}^{2} \operatorname{tr}\left\{\mathbf{A}^{0}\left(\mathbf{A}^{0}\right)^{T}\right\}-\|\beta\|_{\mathcal{I}}^{2} \operatorname{tr}\left\{\mathbf{B}^{0}\left(\mathbf{B}^{0}\right)^{T}\right\}-2 \operatorname{Re}\left[(\alpha, \beta)_{\mathcal{I}} \operatorname{tr}\left\{\mathbf{A}^{0}\left(\mathbf{B}^{0}\right)^{T}\right\}\right] \\
& =-\|\alpha\|_{\mathcal{I}}^{2} \sum_{n} \sum_{x \in K_{n}} A_{x}^{0}-\|\beta\|_{\mathcal{I}}^{2} \sum_{n} \sum_{y \in K_{n}} B_{y}^{0}-2 \operatorname{Re}\left[(\alpha, \beta)_{\mathcal{I}}\right] \sum_{n} \sum_{x \in K_{n}} A_{x}^{0} B_{x}^{0}
\end{aligned}
$$

where $\|\cdot\|_{\mathcal{I}}^{2}$ is the norm coming from the inner product $(\cdot, \cdot)_{\mathcal{I}}$. Also, in the last expression we have used the fact that $\left(\left\{A_{x}^{0}\right\}_{x \in \mathcal{I}}\right)^{\dagger}=\left(\left\{A_{x}^{0}\right\}_{x \in \mathcal{I}}\right)^{T}$, similarly $\left(\left\{B_{y}^{0}\right\}_{y \in \mathcal{I}}\right)^{\dagger}=$ $\left(\left\{B_{y}^{0}\right\}_{y \in \mathcal{I}}\right)^{T}$ as well as the relations of Lemma 4.1.11.

We can see that the algebraic properties of Hamiltonian operator $H_{\alpha, \beta}$ (4.5) are well behaved with respect to the other operators already defined. This is formalized in the following proposition:

Proposition 4.2.1 (Commutation relations for $H_{\alpha, \beta}$ ). The Hamiltonian operator $H_{\alpha, \beta}$ : $\mathbb{C}^{2|\mathcal{I}|} \times \mathcal{H} \rightarrow \mathbb{C}^{2|\mathcal{I}|} \times \mathcal{H}$, defined in equation (4.5), satisfy the following relations:

(i) $H_{\alpha, \beta} A_{x}^{s}=A_{x}^{s} H_{\alpha, \beta}$, for all $x \in K_{n}$ and $s \in \hat{G}_{n+1}$,

(ii) $H_{\alpha, \beta} B_{y}^{h}=B_{y}^{h} H_{\alpha, \beta}$, for all $y \in K_{m}$ and $u \in G_{m-1}$.

where $A_{x}^{s}$ and $B_{y}^{h}$ are as in Definition 4.1.10.

Proof. The relations (i) and (ii) are immediate by consequence of Lemmas 4.1.7 and 4.1.11. 
For the sake of definiteness, we restrict our study to the subclass of Hamiltonian operators defined by the relation

$$
H:=\left\{H_{\alpha, \beta} \mid\|\alpha\|_{\mathcal{I}}^{2}=\|\beta\|_{\mathcal{I}}^{2}=1, \operatorname{Re}\left[(\alpha, \beta)_{\mathcal{I}}\right]=0\right\},
$$

which can be proven to have the topological order expected, as well as reproducing the QDM models. However, the parametrized Hamiltonian of equation (4.5) will be studied in future works.

We formalize last comment by defining:

Definition 4.2.2 (Hamiltonian Operator). We define the Hamiltonian operator $H$ : $\mathcal{H} \rightarrow \mathcal{H}$ to be:

$$
H:=-\sum_{n} \sum_{x \in K_{n}} A_{x}^{0}-\sum_{n} \sum_{y \in K_{n}} B_{y}^{0}
$$

where $A_{x}^{0}$ and $B_{y}^{0}$ are as in Definition 4.1.10.

The above Hamiltonian consists on terms of two kind. The first, $A_{x}^{0}$ is an equal weight sum of local gauge transformations. The second kind of operators, $B_{x}^{0}$ are actually projectors to the trivial $n$-flux sector. It is clear that both set of operators are gauge invariant operators, which is evident from Lemma 4.1.11. As we mentioned earlier, targeting to reproduce several of the models in display at the literature we take these sets of parameters for $\alpha$ and $\beta$ in order to tailor this Hamiltonian in the fashion of the Quantum Double Models. Notice that Proposition 4.2.1 is still valid, as well that the fact that by Lemmas 4.1.7 and 4.1.11, each of the components of the Hamiltonian $H$ commute with one another.

\subsection{Examples}

The following example show that the current construction is a generalization of the Abelian Quantum Double Models beyond the traditional 1-gauge. In fact, a remarkable feature of the formalism presented is that (abelian) gauge transformations of any order can be treated all at once.

\subsubsection{Abelian 1-gauge: Quantum Double Models}

Let $X$ be a compact 2-manifold. The latter can be understood as a complex $\left(C, \partial^{C}\right)$ such that $C=C(X)$ is a simplicial complex with $\partial^{C}$ the usual boundary operator. 


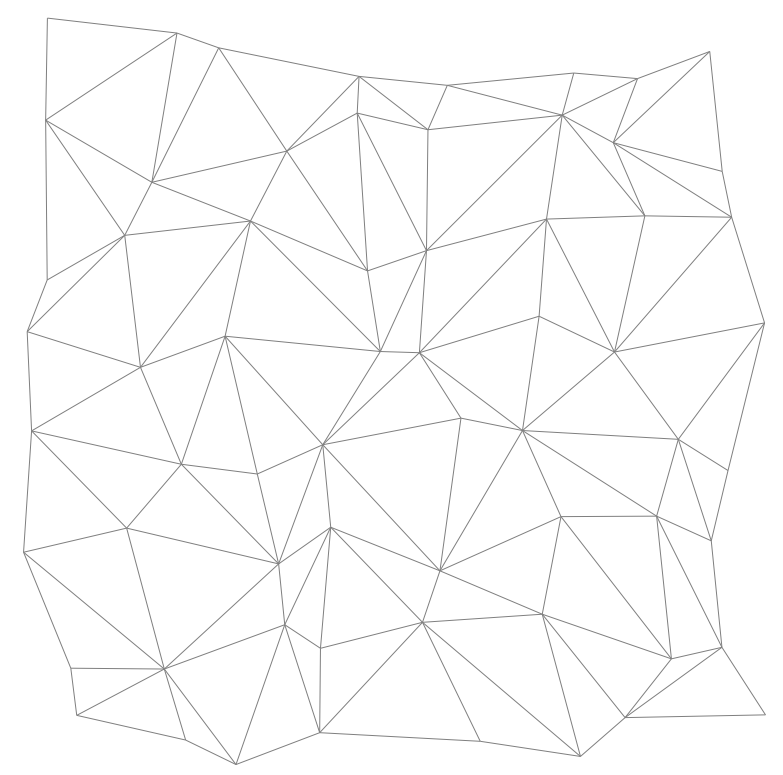

FIGURE 4.2: A triangulated section of a 2-manifold

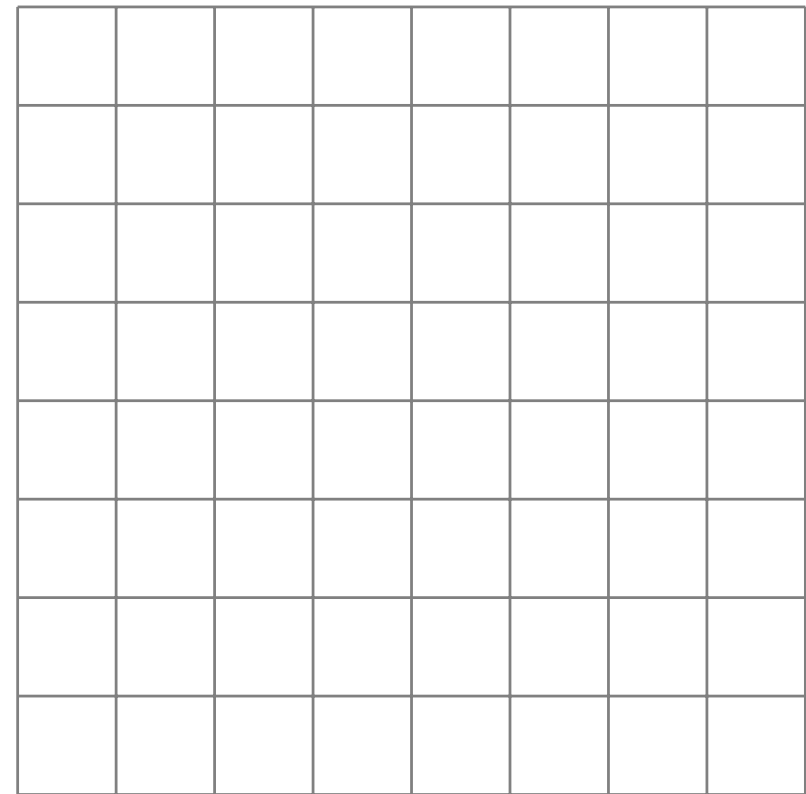

Figure 4.3: A regular squared section of a 2-manifold

It follows that we have the exact chain:

$$
0 \stackrel{\partial_{3}}{\longrightarrow} C_{2} \stackrel{\partial_{2}^{C}}{\longrightarrow} C_{1} \stackrel{\partial_{1}^{C}}{\longrightarrow} C_{0} \stackrel{\partial_{0}^{C}}{\longrightarrow} 0 .
$$

It is easy to show that, in the case of restricting $\left(G, \partial^{G}\right)$ to be the chain

$$
\ldots \rightarrow 0 \stackrel{\partial_{2}^{G}}{\longrightarrow} G_{1} \stackrel{\partial_{1}^{G}}{\longrightarrow} 0 \rightarrow \ldots,
$$

i.e. having only the 1-gauge, we recover the well known Abelian Quantum Double Models. Without loss of generality, instead of considering a triangulated section as shown 
in FIG. 4.2 we can take the simplicial complex $C(X)$ to be square lattice embedded in $X$ with section as shown in FIG. 4.3. In fact, from Definition 4.1.1, the total Hilbert space reads

$$
\mathcal{H}_{\mathrm{QDM}}=\bigotimes_{x \in K_{1}} \mathbb{C}\left[G_{1}\right]=\bigotimes_{l \in C(X)} \mathbb{C}\left[G_{1}\right]
$$

where the last equality follows from recognizing that each $x \in K_{1}$ is associated now to only one link $l$ of $C(X)$, as it was expected.

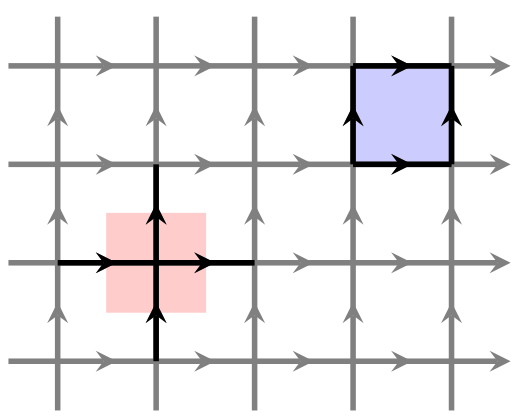

FIgURE 4.4: Planar section of a oriented squared lattice $C(X)$ giving support to the QDM, with the pink and cyan sectors related to local the gauge $A_{g x^{*}}$ and local holonomy $B_{r y_{*}}$ operators, respectively.

$$
A_{g x^{*}}|d \underset{\substack{\uparrow \\
c}}{\stackrel{\uparrow}{\uparrow}}-b\rangle=\left|\begin{array}{c}
a+g \\
d-g \rightarrow \underset{\uparrow}{\uparrow}-g+g
\end{array}\right\rangle
$$

Figure 4.5: Definition of the local operators $A_{g x^{*}}$ and $B_{h y_{*}}$ with relation to the support regions depicted in FIG. 4.4. In this case $x \in K_{0}$ (i.e. vertices) and $y \in K_{2}$ (i.e. plaquetes) of a regular square lattice $C(X)$, with $a, b, c, d, g \in G_{1}$ and $h \in \hat{G}_{1}$.

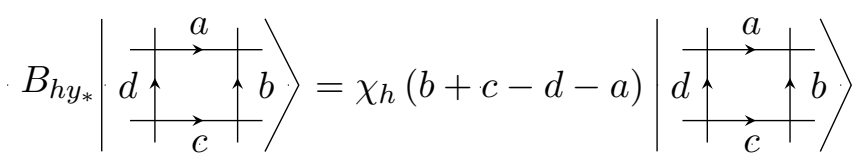

Figure 4.6: Definition of the local operators $A_{g x^{*}}$ and $B_{h y_{*}}$ with relation to the support regions depicted in FIG. 4.4. In this case $x \in K_{0}$ (i.e. vertices) and $y \in K_{2}$

(i.e. plaquetes) of a regular square lattice $C(X)$, with $a, b, c, d, g \in G_{1}$ and $h \in \hat{G}_{1}$.

Furthermore, from Definition 4.1.10, the realization of the operators of the model over the square lattice $C(X)$ involve the regions of support appearing in FIG. 4.4, while the operators are defined as those appearing FIG. 4.6. The latter which coincide with those of the Abelian Quantum Double Models, as claimed. The last identification comes from Definition 4.2.2, from which the Hamiltonian operator reads

$$
\begin{aligned}
H_{\mathrm{QDM}} & =-\sum_{x \in K_{0}} A_{x}^{0}-\sum_{y \in K_{2}} B_{y}^{0} \\
& =-\sum_{v \in \mathcal{L}} A_{v}-\sum_{p \in \mathcal{L}} B_{p} .
\end{aligned}
$$


where, we have recognized that each $x \in K_{0}$ is associated to a vertex $v \in \mathcal{L}$ and each $y \in K_{2}$ is associated to a plaquette $p \in \mathcal{L}$. As claimed.

\subsubsection{Abelian 1,2-gauge theories}

This example needs a bit of background. We warn the reader that we will sacrifice formality for the sake of keeping the length of the example, and hence this paper, short. We begin by paraphrasing the following results found in [56, 74]: Any 2-group completely defines a 2-gauge theory on a smooth compact manifold $X$ (we refer to $[55,56]$ for definitions and explicit constructions on 2-groups, since they are outside of the scope of this paper). On the other hand, there is an explicit equivalence between 2-groups and what is known as a crossed module (defined below). We focus on the latter since they are easier to connect with our current formalism.

We start by considering a compact $n$-manifold such that the complexes $\left(C, \partial^{C}\right)$ with $C=C(X),\left(G, \partial^{G}\right)$ and $f \in \operatorname{hom}(C, G)_{0}$ are as depicted in FIG. 4.7. We have taken $G_{i}=0$ for all $i \neq 1,2$ so we can readily see that $f_{0}$ and $f_{k}$, with $k \geq 3$, do not affect the region of the lower chain between $G_{1}$ and $G_{2}$.

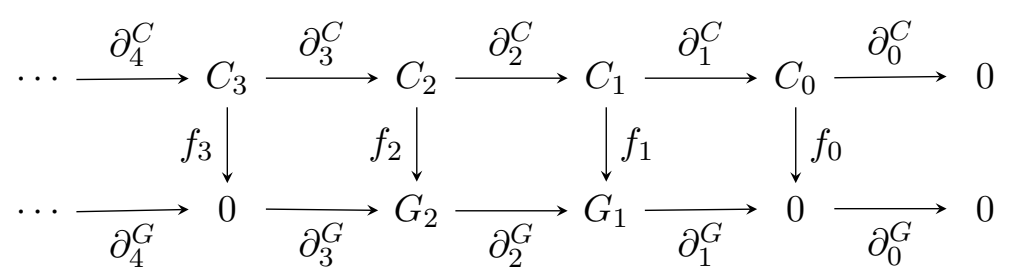

FIGURE 4.7

Under these circumstances, we can recognize the chain $\left(G, \partial^{G}\right)$ to be effectively a crossed module of groups, which is defined as the quadruple $\left(G_{2}, G_{1}, \partial_{2}^{G}, \triangleleft\right)$, where $\triangleleft$ is an action of $G_{1}$ on $G_{2}$ by automorphisms; such that (i) $\partial_{2}^{G}$ is $G_{1}$-equivariant: $\partial_{2}^{G}(h \triangleleft g)=g^{-1} \partial_{2}^{G}(h) g$ for all $g \in G_{1}$ and $h \in G_{2}$, and (ii) $\partial_{2}^{G}$ satisfies the Peiffer identity: $h^{\prime} \triangleleft \partial_{2}^{G}(h)=h^{-1} h^{\prime} h$ in $G_{2}$ for all $h, h^{\prime} \in G_{2}$. Notice that, in our case, the action $\triangleleft$ is innocuous, since the previous requirements are trivialized due to $G_{2}$ and $G_{1}$ being abelian groups.

The latter identification relates the chain of FIG. 4.7 with a 2-gauge abelian theory since when a crossed module is well defined, a 2-gauge theory is also well defined via the equivalence through the corresponding 2-group ( For details see [55, 56, 74]), as claimed. 


\section{Chapter 5}

\section{Ground State Degeneracy for higher abelian gauge theories}

Keeping the rigourous tone from the previous chapter, this one focus on the properties of the ground states of the class of models presented in Chapter 4 and their relation with the topology of the underlying $\operatorname{hom}(C, G)$ complex. This result, is the main feature of the work and is presented in the form of Theorem 5.2.3.

However, before embarking into this topic, we will recall certain considerations concerning a suitable characterization of the ground state subspace $\mathcal{H}_{0} \subset \mathcal{H}$. In fact, the condition was already presented when the QDM were presented in Chapter 3. It is somewhat immediate from the Definition 4.2.2, that the ground state subspace $\mathcal{H}_{0}$ is given by all $|\Psi\rangle$ for which the condition:

$$
\left.\mathcal{H}_{0}:=\left\{|\Psi\rangle \in \mathcal{H}\left|\forall x \quad A_{x}^{0}\right| \Psi\right\rangle=|\Psi\rangle \text { and } B_{x}^{0}|\Psi\rangle=|\Psi\rangle\right\}
$$

holds.

It is easy to probve that the space $\mathcal{H}_{0}$ is non empty, therefore, the system defined by the Hamiltonian (4.2.2) is a frustration free one. This is ensured by the existence of the state $\left|0_{G}\right\rangle$, which will be defined below. Nonetheless, by Remark 4.1.4, this study can be posed entirely in terms of relations involving the operators defined above only.

\subsection{Projector Operators and Projection into $\mathcal{H}_{0}$}

In order to study the ground state subspace we discuss two more sets of operators that will be used to describe the ground states and characterize them: 
Definition 5.1.1 (Generalized projector operators). Given $s \in \widehat{\operatorname{hom}}(C, G)_{-1}, h \in$ $\operatorname{hom}(C, G)_{1}$ we define

$$
\Pi_{A^{s}}:=\prod_{n} \prod_{x \in K_{n}} A_{x}^{s_{n}(x)}, \quad \Pi_{B^{h}}:=\prod_{n} \prod_{x \in K_{n}} B_{x}^{h_{n}(x)},
$$

where the operators $A_{x}^{s_{n}(x)}$ and $B_{x}^{h_{n}(x)}$ are as in Definition 4.1 .10 and $t_{n}(x) \in G_{n-1}$, $s_{n}(x) \in \hat{G}_{n+1}$ are defined in equations (4.2) and (4.4), respectively.

To understand how these generalized projector operators work, we need to prove the following propositions first:

Proposition 5.1.2. Given $s \in \widehat{h o m}(C, G)_{-1}$ and $h \in h o m(C, G)_{1}$ the following identities are satisfied:

$$
\begin{aligned}
& \prod_{n} \prod_{x \in K_{n}} \sum_{g \in G_{n+1}} \chi_{s_{n}(x)}(g) A_{g x^{*}}=\sum_{t} \chi_{s}(t) A_{t}, \\
& \prod_{n} \prod_{x \in K_{n}} \sum_{r \in \hat{G}_{n-1}} \chi_{r}\left(h_{n}(x)\right) B_{r x^{*}}=\sum_{q} \chi_{q}(h) B_{q},
\end{aligned}
$$

where the sums run for all $t \in \operatorname{hom}(C, G)_{-1}$ and $q \in \widehat{\operatorname{hom}}(C, G)_{1}$, respectively.

Proof. Consider the product of the local operators $A_{g x^{*}}$ in the following form:

$$
\begin{aligned}
\prod_{n} \prod_{x \in K_{n}} \sum_{g \in G_{n+1}} \chi_{s_{n}(x)}(g) A_{g x^{*}} & =\sum_{g_{x}} \prod_{n} \prod_{x \in K_{n}} \chi_{s_{n}(x)}\left(g_{x}\right) A_{g_{x} x^{*}} \\
& =\sum_{g_{x}}\left(\prod_{n} \prod_{x \in K_{n}} \chi_{s_{n}(x)}\left(g_{x}\right)\right)\left(\prod_{n} \prod_{x \in K_{n}} A_{g_{x} x^{*}}\right) \\
& =\sum_{g_{x}} \chi_{s}\left(\sum_{n} \sum_{x \in K_{n}} g_{x} x^{*}\right) A_{\sum_{n} \sum_{x \in K_{n}} g_{x} x^{*}} \\
& =\sum_{t} \chi_{s}(t) A_{t},
\end{aligned}
$$

where we have noticed that $\chi_{s_{n}(x)}\left(g_{x}\right)=\chi_{s}\left(g_{x} x^{*}\right)$. The expression is obtained by defining $t:=\sum_{n, x} g_{x} x^{*}$ and realizing that summing over all $t \in \operatorname{hom}(C, G)_{-1}$ is equivalent to summing over all possible values of $g_{x}$. 
Similarly

$$
\begin{aligned}
\prod_{n} \prod_{x \in K_{n}} \sum_{r \in G_{n-1}} \chi_{r}\left(h_{n}(x)\right) B_{r x^{*}} & =\sum_{r_{x}} \prod_{n} \prod_{x \in K_{n}} \chi_{r_{x}}\left(h_{n}(x)\right) B_{r_{x} x^{*}} \\
& =\sum_{r_{x}}\left(\prod_{n} \prod_{x \in K_{n}} \chi_{r_{x}}\left(h_{n}(x)\right)\right)\left(\prod_{n} \prod_{x \in K_{n}} B_{r_{x} x^{*}}\right) \\
& =\sum_{r_{x}} \chi_{\sum_{n} \sum_{x \in K_{n}} r_{x} x_{*}}(h) B_{\sum_{n} \sum_{x \in K_{n}} r_{x} x^{*}} \\
& =\sum_{q} \chi_{q}(h) B_{q},
\end{aligned}
$$

where we have used $\chi_{r_{x}}\left(h_{n}(x)\right)=\chi_{r_{x} x_{*}}(h)$ and defined $q:=\sum_{n, x} r_{x} x_{*} \in \widehat{\operatorname{hom}}(C, G)_{1}$ in the rhs. The expression follows when realizing that summing over all $q \in \widehat{\operatorname{hom}}(C, G)_{1}$ is equivalent to summing over all possible values of $r_{x}$.

Hence, we have the following characterization:

Proposition 5.1.3 (Decomposition of the generalized Projector Operators). The operators $\Pi_{A^{s}}$ and $\Pi_{B^{h}}$, as defined 5.1.1, have the following decompositions:

$$
\Pi_{A^{s}}=\frac{1}{\left|h o m(C, G)_{-1}\right|} \sum_{t} \chi_{s}(t) A_{t} \quad, \quad \Pi_{B^{h}}=\frac{1}{\left|\widehat{h o m}(C, G)_{1}\right|} \sum_{q} \chi_{q}(h) B_{q},
$$

where the sums run for all $t \in \operatorname{hom}(C, G)_{-1}$ and $q \in \widehat{h o m}(C, G)_{1}$, respectively.

Proof. The result follows from Proposition 5.1 .2 by noticing that:

$$
\left|\operatorname{hom}(C, G)_{-1}\right|=\left.\prod_{n}\left|G_{n+1}\right|^{\left|K_{n}\right|} \quad \widehat{\operatorname{hom}}(C, G)_{1}\left|=\prod_{n}\right| G_{n-1}\right|^{\left|K_{n}\right|}
$$

Remark 5.1.4 (Expansion for the ground state projector). In particular, from Proposition 5.1.3 we also have the following useful identities:

$$
\Pi_{A^{0}}=\frac{1}{\left|\operatorname{hom}(C, G)_{-1}\right|} \sum_{t} A_{t}, \quad \Pi_{B^{0}}=\frac{1}{\left|\widehat{\operatorname{hom}}(C, G)_{1}\right|} \sum_{p} B_{p}
$$

So, when using the decompositions shown in Proposition 5.1.3 and, in an analogous way to that of Lemma 4.1.11, it is straightforward to show that the generalized projector operators have the following properties: 
Lemma 5.1.5. For all $s, r \in \widehat{h o m}(C, G)_{-1}, h, u \in h o m(C, G)_{1}$, the following relations for the generalized projector operators (Definition 5.1.1) are satisfied:

(i) Pairwise commutation $\Pi_{A^{s}} \Pi_{A^{r}}=\Pi_{A^{r}} \Pi_{A^{s}} ; \quad \Pi_{B^{h}} \Pi_{B^{u}}=\Pi_{B^{u}} \Pi_{B^{h}} ; \quad \Pi_{A^{s}} \Pi_{B^{u}}=$ $\Pi_{B^{u}} \Pi_{A^{s}}$.

(ii) $\Pi_{A^{s}} \Pi_{A^{r}}=\delta(s, r) \Pi_{A^{s}}, \quad \Pi_{B^{h}} \Pi_{B^{u}}=\delta(h, u) \Pi_{B^{h}}$. Where $\delta(h, u)$ is the Kronecker delta

(iii) $\sum_{s} \Pi_{A^{s}}=i d, \quad \sum_{h} \Pi_{B^{h}}=i d$. Where id is the identity operator.

The main idea is that, by using the previous projector operators, we can show that the Hamiltonian defined in 4.2.2 is a frustration free Hamiltonian. We do this by studying the following particularization of generalized the projector operators of Definition 5.1.1:

Definition 5.1.6 (Ground state projector operator). The operator $\Pi_{0}: \mathcal{H} \rightarrow \mathcal{H}_{0}$, defined as

$$
\Pi_{0}:=\left.\left.\Pi_{A^{s}}\right|_{s=0} \cdot \Pi_{B^{h}}\right|_{h=0},
$$

is called the ground state projector operator, where $\Pi_{A^{s}}$ and $\Pi_{B^{h}}$ are as in Definition 5.1 .1 .

Remark 5.1.7. For brevity, we will write $\left.\Pi_{A^{s}}\right|_{s=0}=\Pi_{A^{0}}$ and $\left.\Pi_{B^{h}}\right|_{h=0}=\Pi_{B^{0}}$ from now on.

Some comments are appropriate at this point; it is clear that $\Pi_{0}$ is indeed a projector operator as the name suggests, this follows easily from Lemma 4.1.11 (ii). The fact that $\Pi_{0}$ projects into the ground state space $\mathcal{H}_{0}$ follows from the additive form of the Hamiltonian 4.2.2. Indeed, it is easy to show that:

$$
H \Pi_{0}|\Psi\rangle=\Pi_{0} H|\Psi\rangle \quad \Leftrightarrow \quad|\Psi\rangle \in \mathcal{H}_{0}
$$

which establishes the equivalence between the two characterizations of the ground state.

It is also worth mentioning that the operators $\left.\Pi_{A^{s}}\right|_{s \neq 0}$ and $\left.\Pi_{B^{h}}\right|_{h \neq 0}$ project the Hilbert space into the excited state subspaces labeled by the maps $\left\{s \in \operatorname{hom}(C, G)_{-1} \mid s \neq 0\right\}$ and $\left.\{h \in \widehat{\operatorname{hom}(C,} G)_{1} \mid h \neq 0\right\}$ that correspond to the charge and flux sectors, respectively. A detailed study of the excited state subspace is out of the scope of this paper but will be presented in a forthcoming work. 


\subsubsection{Gauge Equivalence}

The aim of this section is to show that we can interpret the product $\prod_{A^{0}}$ as being an operator projecting states into the subspace of states which are gauge equivalent. To make this last assertion precise we first define what will be understood for gauge equivalence and gauge invariance in these models. We move away from the geometrical interpretation by the reasons we have stated before, i.e. there is no clear geometrical interpretation of the maps $f \in \operatorname{hom}(C, G)_{p}$, and we rather take the approach of defining it through the related concept of recognizing an equivalence of maps with respect to the $\delta_{p}$ operation as follows:

Definition 5.1.8 (Gauge equivalence). Two states $|f\rangle,|g\rangle \in \mathcal{H}$ are gauge equivalent if there is some $t \in \operatorname{hom}(C, G)_{-1}$ such that $A_{t}|f\rangle=|g\rangle$ or, equivalently, that $g=f+\delta_{-1} t$.

We leave it to the reader to prove that the expression $g=f+\delta_{-1} t$ defines an equivalence class since it follows immediately from the definition of the cohomology (hom $(C, G), \delta$ ). However, it must be stressed that this notion of gauge equivalence is more general than that of the usual gauge theories. This is due to the nature of the equivalence class defined in 5.1.8, in terms of the map $\delta_{-1}: \operatorname{hom}(C, G)_{-1} \rightarrow \operatorname{hom}(C, G)_{0}$. Therefore, we have:

Lemma 5.1.9 (Gauge equivalent base elements). Definition 5.1.8 is equivalent to the statement that two basis states $|f\rangle,|g\rangle \in \mathcal{H}$ are gauge equivalent if and only if $\Pi_{A^{0}}|f\rangle=$ $\Pi_{A^{0}}|g\rangle$.

Proof. It is straight forward to check that (See Remark 5.1.4)

$$
\Pi_{A^{0}}=\frac{1}{\left|\operatorname{hom}(C, G)_{-1}\right|} \sum_{t} A_{t} \quad, \quad \forall t \in \operatorname{hom}(C, G)_{-1}
$$

so, by the previous Definition 5.1.8, $\Pi_{A}$ maps any basis state $|f\rangle \in \mathcal{H}$ into the superposition of all gauge equivalent states. The result follows directly from this remark.

\subsection{The Ground State Degeneracy Theorem}

Lemma 5.1.9 allows us to understand the canonical state $\left|0_{G}\right\rangle=\Pi_{A^{0}}|0\rangle \in \mathcal{H}_{0}$ as a superposition of all basis states that are gauge equivalent to $|0\rangle$, the flux-free product state. Moreover, suppose we have

$$
\begin{aligned}
\left|f_{G}\right\rangle & :=\Pi_{A^{0}}|f\rangle \\
& =P_{f}\left|0_{G}\right\rangle \in \mathcal{H}_{0},
\end{aligned}
$$


since $\Pi_{A^{0}}$ commutes with $P_{f}$. This state is then understood merely as a superposition of gauge equivalent states to that of $|f\rangle$, then, by equation (5.1) and Proposition 4.1.7 we have:

$$
\begin{aligned}
\left|f_{G}\right\rangle & =\Pi_{0}\left|f_{G}\right\rangle \\
& =\Pi_{A^{0}} \Pi_{B^{0}} P_{f} \Pi_{A^{0}}|0\rangle \\
& =\Pi_{B^{0}} P_{f}\left|0_{G}\right\rangle \\
& =\Pi_{B^{0}}\left|f_{G}\right\rangle,
\end{aligned}
$$

where we have used Remark 4.1.4 repeatedly. The latter observation leads to the following Proposition.

Proposition 5.2.1. Let $f \in \operatorname{hom}(C, G)_{0}$. The state $\left|f_{G}\right\rangle=\Pi_{A^{0}}|f\rangle$, belongs to the ground state space $\mathcal{H}_{0}$ if, and only if, $f \in \operatorname{ker}\left(\delta_{0}\right)$.

Proof. Notice that, by the previous discussion, $\left|f_{G}\right\rangle \in \mathcal{H}_{0}$ implies $\left|f_{G}\right\rangle=\Pi_{B^{0}}\left|f_{G}\right\rangle$. When using the expansion of Remark 5.1.4 and Remark 4.1.4 we have:

$$
\begin{aligned}
\left|f_{G}\right\rangle & =\frac{1}{\left|\widehat{\operatorname{hom}}(C, G)_{1}\right|} \sum_{s} B_{s} P_{f}\left|0_{G}\right\rangle \\
& =\frac{1}{\left|\widehat{\operatorname{hom}}(C, G)_{1}\right|} \sum_{s} \chi_{\hat{\delta}_{1}(s)}(f) P_{f} B_{s}\left|0_{G}\right\rangle \\
& =\left(\frac{1}{\left|\widehat{\operatorname{hom}}(C, G)_{1}\right|} \sum_{s} \chi_{s}\left(\delta_{0}(f)\right)\right)\left|f_{G}\right\rangle
\end{aligned}
$$

where we have used the fact that $B_{s}$ is diagonal over $\left|0_{G}\right\rangle$. The last step also follows from using the dual morphism $\hat{\delta}_{1}$. The Proposition then holds when using the orthonormal relations for the characters. This forces $\delta_{0} f=0$, or equivalently $f \in \operatorname{ker}\left(\delta_{0}\right)$.

The above proposition characterizes the ground state subspace. This is, any state $\left|f_{G}\right\rangle$ with $f \in \operatorname{ker}\left(\delta_{0}\right)$ is a ground state of the model. However, to determine the ground state degeneracy of the model we need to characterize the basis states of the ground state subspace. This is done by noticing that the states $\left|f_{G}\right\rangle$ with $f \in \operatorname{ker}\left(\delta_{0}\right)$ are in correspondence with the equivalence classes of the cohomology group $H^{0}(C, G)$ modulo gauge equivalence, i.e.

Proposition 5.2.2. The states $\left\{\left|f_{G}\right\rangle \mid f \in \operatorname{ker}\left(\delta_{0}\right)\right\}$ are a basis for the ground state subspace and are in one-to-one correspondence with elements of $H^{0}(C, G)$.

Proof. It is clear from Proposition 5.2.1 that $\left|f_{G}\right\rangle \in \mathcal{H}_{0}$ if, and only if, $f \in \operatorname{ker}\left(\delta_{0}\right)$ and Lemma 5.1 .9 implies $\left|f_{G}\right\rangle=\left|g_{G}\right\rangle$ if, and only if, $f-g \in \operatorname{im}\left(\delta_{-1}\right)$. Therefore, one can 
assign a equivalence class $[f] \in H^{0}(C, G)=\operatorname{ker}\left(\delta_{0}\right) / \operatorname{im}\left(\delta_{-1}\right)$ to each state $\left|f_{G}\right\rangle$ in a well defined manner and obtain a bijection between $\left\{\left|f_{G}\right\rangle \mid f \in \operatorname{ker}\left(\delta_{0}\right)\right\}$ and $H^{0}(C, G)$. One can then project the basis $\{|f\rangle\}$ of $\mathcal{H}$ into a basis of $\mathcal{H}_{0}$ using $\Pi_{0}$. The outcome is precisely $\left.\left.\left\{\Pi_{0}|f\rangle\left|\Pi_{0}\right| f\right\rangle \neq 0\right\}=\left\{\left|f_{G}\right\rangle\left|\Pi_{B^{0}}\right| f_{G}\right\rangle \neq 0\right\}=\left\{\left|f_{G}\right\rangle \mid f \in \operatorname{ker}\left(\delta_{0}\right)\right\}$ so the result follows.

Furthermore, proposition 5.2.2 also implies that, for each cohomology class $[f] \in H^{0}(C, G)$, there is a well defined operator

$$
P_{[f]}:=\prod_{g \sim f} P_{g}=P_{f} \Pi_{A^{0}}
$$

that creates the ground states from the $|0\rangle$ state, this last comment allows us to state the main result:

Given a finite simplicial complex $C$, a chain complex of finite abelian groups $\left(G_{m}, \partial^{G}\right)$ and $\mathcal{H}$ the Hilbert space of definition 4.1 .1 with Hamiltonian $H: \mathcal{H} \rightarrow \mathcal{H}$ as defined in 4.2.2. The following theorem follows:

Theorem 5.2.3 (Dimension of the ground state subspace). The dimension of the ground state subspace $\mathcal{H}_{0}$ is given by:

$$
G S D=\left|H^{0}(C, G)\right| \cong \prod_{n}\left|H^{n}\left(C, H_{n}(G)\right)\right|
$$

Proof. The proof follows immediately from Propositions 5.2.1 and 5.2.2, as well as Theorem C.2.6.

In physical terms, Theorem 5.2.3 underscores a very useful way to understand the GSD; this is, there is a contribution to it from each individual cohomology $H^{n}\left(C, H_{n}(G)\right)$. Moreover, intricate relations between geometrical quantities (related to the $C$ complex) and gauge quantities (related to the $G$ complex) can be present. Let us exemplify the latter by restricting $C$ as coming from a closed triangulable manifolds. This allows us to use the universal coefficient theorem (See Appendix $\mathrm{C}$ or [75] for a general reference), such that we can decompose each contribution as:

$$
H^{n}\left(C, H_{n}(G)\right)=\operatorname{Hom}\left(H_{n}(C), H_{n}(G)\right) \oplus \operatorname{Ext}^{1}\left(H_{n-1}(C), H_{n}(G)\right)
$$

for all $0 \leq n \leq d$. Here is explicit that, if the $G$ complex is not changed, any two homological triangulable manifolds $\left(X_{1} \cong X_{2} \Rightarrow C\left(X_{1}\right)=C\left(X_{2}\right)\right)$ will have the same GSD. On the other hand, the appearance of two different homologies $\left(H_{n}(C)\right.$ and $\left.H_{n-1}(C)\right)$ 
in the decomposition (5.3), makes the physical interpretation of the terms somewhat cumbersome since it calls for a case by case study.

\subsection{Examples}

This section will make the connection with the abelian quantum doubles as well as other higher gauge theory in its abelian case clear. Notice the power of the theorem 5.2.3. It basically says that knowing the geometry ( $C$ complex) as well as knowing the graded group ( $G$ complex) is enough information to calculate the GSD of the models.

\subsubsection{GSD of the Toric code}

Within the conditions discussed above we can take the example of the Toric Code, proposed by Kitaev [24, 27, 76]. Let $C=C\left(T^{2}\right)$ be a regular squared lattice embedded over a Torus $T^{2}$ as shown in FIG. 5.1.

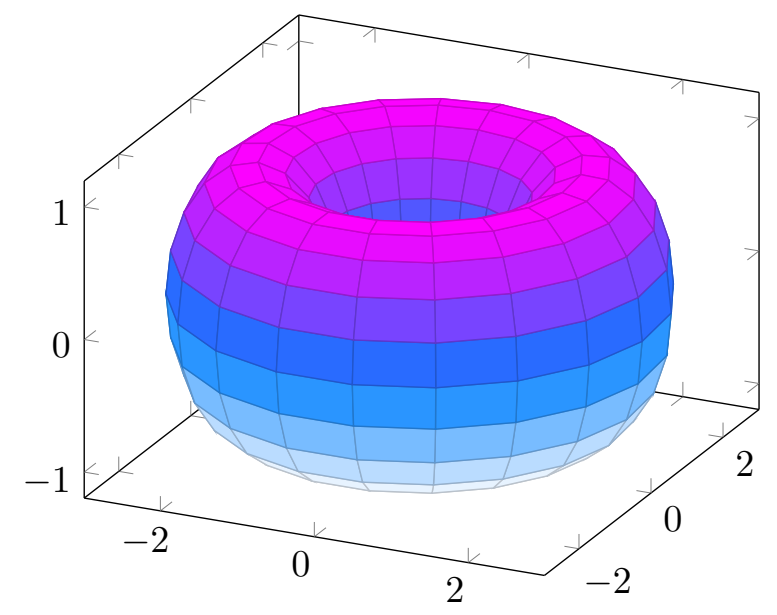

Figure 5.1: Regular squared lattice embedded over a Torus $T^{2}$

The toric code, can then be understood as a particular case of the quantum double models of Example 4.3.1 for which $G_{1}=\mathbb{Z}_{2}$. We then have the hom $(C, G)_{0}$ to be of the form shown in FIG. 5.2 with associated homology groups shown in FIG. 5.3.

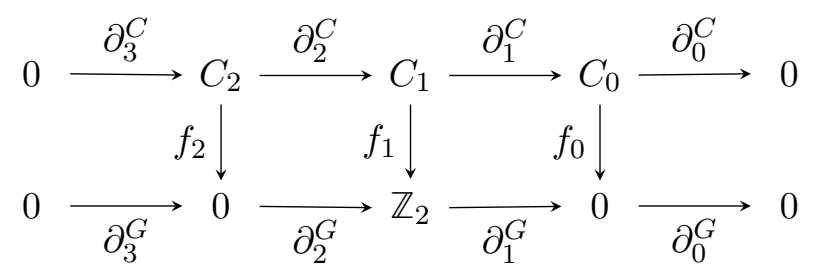

Figure 5.2: $\operatorname{hom}(C, G)_{0}$ for the toric code. 


$$
H_{n}(C) \cong H_{n}\left(T^{2}\right)=\left\{\begin{array}{ll}
\mathbb{Z}, & n=0, \\
\mathbb{Z} \oplus \mathbb{Z}, & n=1, \\
\mathbb{Z}, & n=2 .
\end{array} \quad ; H_{n}(G)= \begin{cases}0, & n=0 \\
\mathbb{Z}_{2}, & n=1 \\
0, & n=2 .\end{cases}\right.
$$

Figure 5.3: Homology groups for the hom $(C, G)_{0}$ of the toric code.

We can use Theorem 5.2.3 to obtain the ground state degeneracy of this model as follows:

$$
\operatorname{GSD}=\left|H^{0}(C, G)\right|=\left|H^{1}\left(C, H_{1}(G)\right)\right|=2^{2},
$$

where we have used the universal coefficient theorem ( Eqn. (5.3) ) in order to expand the terms in $\left|H^{0}(C, G)\right|$. Notice that, as expected, the result coincides with the usual expression for the ground state of the toric code found in the literature [24, 27].

\subsubsection{GSD of the 3D Toric Code on $T^{3}$}

As an additional example we can consider the 3D version of the Toric Code on the 3torus $T^{3}[77]$. The construction is analogous to the one given in the previous example, the geometric simplicial complex is $C=C\left(T^{3}\right)$ and the $G$ complex consists on a single non-trivial piece $G_{1}=\mathbb{Z}_{2}$. The homology groups of $T^{3}$ are given by :

$$
H_{n}(C) \cong H_{n}\left(T^{3}\right)=\left\{\begin{array}{l}
\mathbb{Z}, \quad n=0,3 \\
\mathbb{Z} \oplus \mathbb{Z} \oplus \mathbb{Z}, \quad n=1,2
\end{array} \quad ; \quad H_{n}(G)=\left\{\begin{array}{ll}
0, & n=0 \\
\mathbb{Z}_{2}, & n=1, \\
0, & n=2 .
\end{array} .\right.\right.
$$

Therefore, the ground state degeneracy of the model is:

$$
\operatorname{GSD}=\left|H^{0}(C, G)\right|=\left|H^{1}\left(C, H_{1}(G)\right)\right|=\left|\operatorname{Hom}\left(H_{1}\left(T^{3}\right), H_{1}(G)\right)\right|=2^{3},
$$

as expected [77].

\subsubsection{Abelian 1,2-gauge theory}

In this example we consider a slightly more general situation, namely, a 1,2-gauge theory defined on a discretization of a 2 -sphere $S^{2}$. This is, the 1-gauge degrees of freedom are located at the 1-simplices of $C_{1} \in C\left(S^{2}\right)$ whereas the 2-gauge ones live on the 2simplices of $C_{2} \in C\left(S^{2}\right)$ which is clear from the definition of $\operatorname{hom}(C, G)_{0}$. We take $G_{1}=\mathbb{Z}_{2}=\{1,-1\}$ and $G_{2}=\mathbb{Z}_{4}=\{1, i,-1,-i\}$ and the boundary map is defined by $\partial_{2}^{G}(i)=-1$. The $\operatorname{hom}(C, G)_{0}$ and the homology groups are shown in Figs.5.4 and 5.5, respectively. 


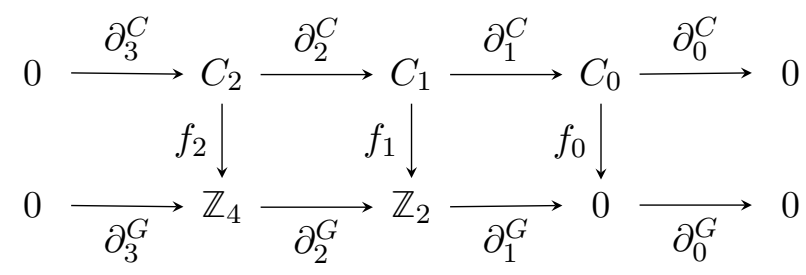

Figure 5.4: $\operatorname{hom}(C, G)_{0}$ for the abelian 1,2-gauge theory.

$$
H_{n}(C) \cong H_{n}\left(S^{2}\right)=\left\{\begin{array}{ll}
0, & n=0, \\
0, & n=1, \\
\mathbb{Z}, & n=2 .
\end{array} \quad ; H_{n}(G)=\left\{\begin{array}{lc}
0, & n=0 \\
0, & n=1, \\
\mathbb{Z}_{2}, & n=2 .
\end{array}\right.\right.
$$

FiguRE 5.5: Homology groups for the abelian 1,2-gauge theory.

By Theorem 5.2.3, the GSD of the abelian 1,2-gauge theory is given by:

$$
G S D=\left|H^{0}(C, G)\right|=\left|H^{2}\left(C, H_{2}(G)\right)\right|=\left|\operatorname{Hom}\left(H_{2}(C), H_{2}(G)\right)\right|=2 .
$$

Thus, the model exhibits degeneracy when defined on the 2-sphere $S^{2}$. However, it can be shown that such topological degeneracy is highly unstable as the two ground states are mapped between each other by local operators (see [51] for a detailed discussion).

\subsubsection{Ground state of abelian 2-gauge and crossed module of racks}

In this example we abandon the rigor in order to briefly show how the ground state condition 5.2.1 can be understood in abelian 2-gauge theory as defining an algebraic structure known as a crossed module of racks (defined below). This example heavily draws from [78] throughout the example.

We need two ingredients to define a crossed module of racks. We start by defining a rack as being a set $C_{i}$ equipped with a binary operation $(x, y) \mapsto x \star_{i} y$ that is invertible and self-distributive, i.e. $\left(x \star_{i} y\right) \star_{i} z=\left(x \star_{i} z\right) \star_{i}\left(y \star_{i} z\right)$ for all $x, y, z \in C_{i}$. The archetype example of a rack's binary operator $\star_{i}$ is the conjugation operator, which in the case of taking $C_{i}$ s to be our free generated abelian groups is trivialized, this is, $x \star_{i} y=x$ for all $x, y \in C_{i}$.

Secondly, given $G_{i}$, we can take $C_{i}$ to be a $G_{i}$-set. We say that $C_{i}$ together with a map $p_{i}: C_{i} \rightarrow G_{i}$ is an augmented rack when it satisfies the augmentation identity, i.e. $p_{i}\left(x \triangleleft_{i} g\right)=g^{-1} p_{i}(x) g$ for all $g \in G_{i}$ and all $x \in C_{i}$. Similarly as before, an augmented rack is, again, trivialized when considering that our $G_{i}$ s are abelian groups, this is, $p_{i}\left(x \triangleleft_{i} g\right)=p_{i}(x)$ for all $g \in G_{i}$. 
We can now define a crossed module of racks as morphisms of racks $\alpha_{i}: C_{i} \rightarrow C_{i-1}$ together with an action $\odot_{i}$ of $C_{i-1}$ on $C_{i}$ by automorphisms such that (i) $\alpha_{i}$ is equivariant, i.e. $\alpha_{i}\left(r \odot_{i} s\right)=\alpha_{i}(r) \star_{i} s$ for all $s \in C_{i-1}, r \in C_{i}$ and (ii) Peiffer's identity is satisfied, i.e. $r \odot_{i} \alpha_{i}\left(r^{\prime}\right)=r \star_{i} r^{\prime}$ for all $r, r^{\prime} \in C_{i}$. As expected, when taking $C_{i}, C_{i-1}$ to be free generated abelian groups we trivialize the previous structures, giving $\alpha_{i}\left(r \odot_{i} s\right)=\alpha_{i}(r)$ for all $s \in C_{i-1}, r \in C_{i}$ and $r \odot_{i} \alpha_{i}\left(r^{\prime}\right)=r$ for all $r, r^{\prime} \in C_{i}$.

In [78] it is shown that these structures can be combined for some commutative diagram as depicted in FIG. 5.6. It follows that we can make the identifications $p_{1}:=f_{1}, p_{2}:=f_{2}$, $\tilde{\partial}_{2}^{G}:=\partial_{2}^{G}$ and $\alpha_{2}:=\partial_{2}^{C}$ if and only if the diagram of FIG. 5.7 is commutative, this is, only when $f \in \operatorname{ker}\left(\delta_{0}\right)$.

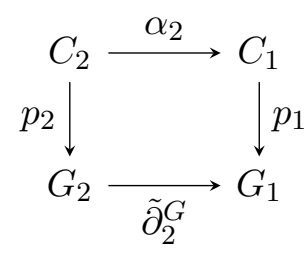

FiguRE 5.6: A crossed module of racks

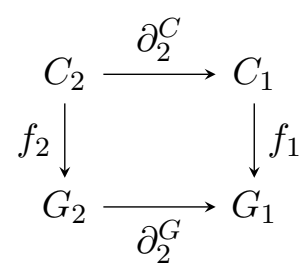

FIGURE 5.7: A ground state of an abelian 2-gauge theory

Figure 5.8

In other words, a ground state of a 2 -gauge abelian theory is determined by all the $f_{1}$, $f_{2}$ and $\partial_{2}^{G}$ that makes FIG. 5.7 commutative, in which case we recognize $\partial_{2}^{C}$ as fulfilling the properties of an abelian crossed module of racks.

\subsection{Characterization of the Ground States}

Theorem 5.2.3 provides an efficient way to compute the ground state degeneracy and operators which can be used to generate all ground states. Nonetheless, it does note give good intuition on the physical properties of the actual states. In this section we hope to remedy this by describing a set of operators that can be used to measure and differentiate each ground state. Moreover, using Theorem C.2.6, a more geometrical description will be provided and enable the definition of suitable quantum numbers for the ground states and a framework for their study. 


\subsubsection{Measurement operators for the space $\mathcal{H}_{0}$}

Let us start by stating that a measurement operator for the ground states $\mathcal{O}: \mathcal{H} \rightarrow$ $\mathcal{H}$ is an operator such that $\mathcal{O}\left|f_{G}\right\rangle=\mathcal{O}([f])\left|f_{G}\right\rangle$ where $[f] \in H^{0}(C, G)$ and $\left|f_{G}\right\rangle=$ $P_{[f]}|0\rangle \in \mathcal{H}_{0}$ is the corresponding ground state. The different eigenvalues $\mathcal{O}([f])$ are used to distinguish ground states so that two measurement operators $\mathcal{O}, \mathcal{O}^{\prime}$ are considered equivalent if $\mathcal{O}([f])=\mathcal{O}^{\prime}([f])$ for all $[f] \in H^{0}(C, G)$.

A natural choice of measuring operators are the clock operators of Definition 4.1.2 since they are diagonal on the basis we have used to construct the ground states. However, the operators that measure ground states are parametrized by special elements of $\widehat{\operatorname{hom}}(C, G)_{0}$, this is:

Proposition 5.4.1. The operator $Q_{m}: \mathcal{H} \rightarrow \mathcal{H}$ defines a measurement operator for the ground state subspace if and only if $m \in \operatorname{ker}\left(\hat{\delta}_{0}\right)$.

Proof. Consider some $f \in \operatorname{ker}\left(\delta_{0}\right)$ and notice that $Q_{m}\left|f_{G}\right\rangle$ can be written as

$$
Q_{m}\left|f_{G}\right\rangle=\frac{1}{\left|\operatorname{hom}(C, G)_{-1}\right|} \sum_{t} Q_{m}\left|f+\delta_{-1} t\right\rangle=\frac{\chi_{m}(f)}{\left|\operatorname{hom}(C, G)_{-1}\right|} \sum_{t} \chi_{m}\left(\delta_{-1} t\right)\left|f+\delta_{-1} t\right\rangle
$$

but $Q_{m}\left|f_{G}\right\rangle$ needs to be proportional to $\left|f_{G}\right\rangle$ and this can only happen if $\chi_{m}\left(\delta_{-1} t\right)=1$ for all $t$. Therefore, $m$ must be trivial on $\operatorname{im}\left(\delta_{-1}\right)$ which is equivalent to $m \in \operatorname{ker}\left(\hat{\delta}_{0}\right)$. A more direct way to prove this is to observe that $Q_{m} \Pi_{A^{0}}=\Pi_{A^{\delta_{0} m}} Q_{m}$ and $\sum_{s} \Pi_{A^{s}}=$ id so $Q_{m}\left|f_{G}\right\rangle=\chi_{m}(f) \Pi_{A^{\hat{\delta}_{0} m}}|f\rangle$ can only be proportional to $\left|f_{G}\right\rangle=\Pi_{A^{0}}|f\rangle$ if $\hat{\delta}_{0} m=0$.

Moreover, if $m, m^{\prime} \in \operatorname{ker}\left(\hat{\delta}_{0}\right)$ satisfy $\left.m\right|_{\operatorname{ker}\left(\delta_{0}\right)}=\left.m^{\prime}\right|_{\operatorname{ker}\left(\delta_{0}\right)}$ then they define the same measurement operator since

$$
\chi_{m^{\prime}}(f)=\left.\chi_{m^{\prime}}\right|_{\operatorname{ker}\left(\delta_{0}\right)}(f)=\chi_{\left.m\right|_{\operatorname{ker}\left(\delta_{0}\right)}}(f)=\chi_{m}(f),
$$

where we've used the fact that $f \in \operatorname{ker}\left(\delta_{0}\right)$ for $[f] \in H^{0}(C, G)$. Thus, the measurement operators are defined up to an equivalence class $[m] \in \operatorname{ker}\left(\hat{\delta}_{0}\right) / \sim$ defined by the relation $m^{\prime} \sim m$ if, and only if, $\left.m^{\prime}\right|_{\operatorname{ker}\left(\delta_{0}\right)}=\left.m\right|_{\operatorname{ker}\left(\delta_{0}\right)}$. Therefore there is a one-to-one correspondence between ground state measurement operators $Q_{m}$ and elements of $\operatorname{ker}\left(\hat{\delta}_{0}\right) / \sim$.

Each class $[m] \in \operatorname{ker}\left(\hat{\delta}_{0}\right) / \sim$ induces a representation $\bar{m} \in \widehat{H^{0}(C, G)}$ defined by $\bar{m}([f])=$ $m(f)$. To see that this is well defined notice that if $m^{\prime} \sim m$ then $m^{\prime}(f)=m(f)$ since $f \in \operatorname{ker}\left(\delta_{0}\right)$ and if $f^{\prime} \sim f$ then $m\left(f^{\prime}\right)=m(f)$ because $f^{\prime}-f \in \operatorname{im}\left(\delta_{-1}\right)$. This procedure defines an isomorphism and it is straightforward to check that the inverse is obtained by mapping $r \in \widehat{H^{0}(C, G)}$ into the equivalence class $[\tilde{r}] \in \operatorname{ker}\left(\hat{\delta}_{0}\right) / \sim$ defined by $\tilde{r}(f)=r([f])$. Finally we arrive at the desired result: 
Proposition 5.4.2. The equivalence classes of ground state measurement operators $Q_{m}$ are in one-to-one correspondence with elements of $\widehat{H^{0}(C, G)}$.

This shouldn't come as a surprise. After all, Theorem 5.2.3 showed that $H^{0}(C, G)$ generates the different ground states so it should be expected that the representation of this groups can be used as observables for the ground states.

\subsubsection{A geometrical description of the ground states}

Theorem 5.2.3 and Proposition 5.4.2 suggest that elements of $H^{0}(C, G)$ can be used as quantum numbers to describe the ground state subspace of the model. However, it is unclear what is the geometrical interpretation behind some $[f] \in H^{0}(C, G)$ since the operator $P_{[f]}$ does a lot of operations simultaneously. In order to obtain such an understanding we are going to use a concrete realization of the isomorphism of Theorem C.2.6.

One such realization is given by the following map:

In [52], restated here in Theorem C.2.6, it is shown that the space $H^{p}(C ; G)$ is isomorphic to $\prod_{n} H^{n}\left(C ; H_{n-p}(G)\right)$. Hence, to build an isomorphism $\iota: \prod_{n} H^{n}\left(C ; H_{n-p}(G)\right) \rightarrow$ $H^{p}(C ; G)$, it is sufficient to build a morphism which is injective. We do this in the following way: let $\pi_{n}: \operatorname{ker}\left(\partial_{n}^{G}\right) \rightarrow H_{n}(G)$ denote the canonical projection, it is then straightforward to check that there are morphisms $\phi_{n}: H_{n}(G) \rightarrow \operatorname{ker}\left(\partial_{n}^{G}\right) \subset G_{n}$ satisfying the relation $\pi_{n} \circ \phi_{n}=$ id. Fix a collection $\phi=\left\{\phi_{n}\right\}_{n}$ of such morphisms.

Next, given $f=\left\{f_{n}: C_{n} \rightarrow H_{n-p}(G)\right\}$, let $\phi f \in \operatorname{hom}(C, G)_{p}$ be defined by

$$
(\phi(f))_{m}=\phi_{m-p} \circ f_{m}
$$

we can calculate $\delta_{p}(\phi(f)):=\phi d(f)$ as follows

$$
\begin{aligned}
\left(\delta_{p}(\phi f)\right)_{m} & =(\phi f)_{m-1} \partial_{m}^{C}-(-1)^{p} \partial_{m-p}^{G}(\phi f)_{m} \\
& =\phi_{m-1-p} f_{m-1} \partial_{m}^{C}-(-1)^{p} \partial_{m-p}^{G} \phi_{m-p} f_{m} \\
& =\phi_{m-1-p} f_{m-1} \partial_{m}^{C} \\
& =\phi_{m-1-p} d_{m-1}^{m-1-p}\left(f_{m-1}\right)
\end{aligned}
$$

where $d(f)$ has components $(d(f))_{m}=d_{m-1}^{m-1-p}\left(f_{m-1}\right)=f_{m-1} \partial_{m}^{C}$ and the last equality holds due to the fact that the image of $\phi_{m-p}$ is contained in $\operatorname{ker}\left(\partial_{m-p}^{G}\right)$, therefore, $\partial_{m-p}^{G} \phi_{m-p}=0$. As a consequence, if every $f_{n}$ belongs to $\operatorname{ker}\left(d_{n}\right)$, then $d(f)=0$ so $\phi(f) \in \operatorname{ker}\left(\delta_{p}\right)$ since $\delta_{p}(\phi f)=\phi d(f)=0$. Moreover, if we pick $g_{n}: C_{n} \rightarrow H_{n+1-p}(G)$ 
then $\phi f+\phi d(g)=\phi f+\delta_{p-1}(\phi g)$. As a result, we can map $\sum\left[f_{n}\right] \in \prod_{n} H^{n}\left(C ; H_{n-p}(G)\right)$ into $[\phi f] \in H^{p}(C ; G)$ in a well defined manner by only choosing a representative $f_{n}$ from each cohomology class $\left[f_{n}\right]$. We formalize the latter in the following Definition:

Definition 5.4.3. The morphism $\iota_{\phi}: \prod_{n} H^{n}\left(C ; H_{n-p}(G)\right) \rightarrow H^{p}(C ; G)$ is defined by

$$
\iota_{\phi}\left(\sum\left[f_{n}\right]\right):=[\phi f]
$$

where $\sum\left[f_{n}\right]$ is an general element of $\prod_{n} H^{n}\left(C ; H_{n-p}(G)\right)$ with $\left[f_{n}\right] \in^{n}\left(C ; H_{n-p}(G)\right)$ and $f$ denotes a collection of maps $f_{n}: C_{n} \rightarrow H_{n-p}(G)$ with $f_{n} \in\left[f_{n}\right]$.

The above discussion implies that this map is well defined, since it is clear that it does not depend on the choice of representatives morphisms. After all, if $f_{n}^{\prime} \in\left[f_{n}\right]$ then there is some $g_{n}$ such that $f^{\prime}=f+d g$ so $\left[\phi f^{\prime}\right]=[\phi f+\phi d g]=\left[\phi f+\delta_{p-1}(\phi g)\right]=[\phi f]$. All that remains is to prove that $\iota_{\phi}$ is injective

Proposition 5.4.4. The morphism $\iota_{\phi}$, defined in 5.4.3, is injective.

Proof. Take $\sum\left[f_{n}\right]$ such that $\iota_{\phi}\left(\sum\left[f_{n}\right]\right)=[\phi f]=[0]$ so that there is some $t \in \operatorname{hom}(C ; G)_{p-1}$ such that $\phi f=\delta_{p-1}(t)$. Therefore,

$$
(\phi f)_{m}=\left(\delta_{p-1}(t)\right)_{m} \quad \Longrightarrow \quad \phi_{m-p} f_{m}=t_{m-1} \partial_{m}^{C}-(-1)^{p-1} \partial_{m-p}^{G} t_{m}
$$

Notice that each image of each term is contained in $\operatorname{ker}\left(\partial_{m-p}^{G}\right)$ so we can compose with $\pi_{m-p}$ to obtain $f_{m}=\pi_{m-p} t_{m-1} \partial_{m}^{C}$ by using $\pi_{m-p} \phi_{m-p}=i d$ and $\pi_{m-p} \partial_{m-p}^{G}=0$. Therefore, $f_{m}=d_{m-1}^{m-p}\left(\pi_{m-p} t_{m-1}\right)$ so $\left[f_{m}\right]=[0]$. We can now conclude that $\sum\left[f_{n}\right]=[0]$ and, hence, $\operatorname{ker}\left(\iota_{\phi}\right)$ is trivial. This is, $\iota_{\phi}$ is injective. as claimed.

By passing, we should also mention that, even though we have used $\phi$ to define $\iota_{\phi}$ the actual morphism does not depend on it. To see this we can choose another collection $\psi$ which also obeys $\pi_{m} \psi_{m}=i d$. Then, $(\phi f-\psi f)_{m}=(\phi-\psi)_{m-p} f_{m}$ but $\pi_{m}(\phi-\psi)_{m}=0$, implying that there is some $\xi$ such that $(\phi-\psi)_{m}=\partial_{m}^{G} \xi_{m}$, therefore

$$
\begin{aligned}
(\phi f-\psi f)_{m} & =\partial_{m-p}^{G} \xi_{m-p} f_{m} \\
& =(-1)^{p}\left(\xi_{m-1-p} f_{m-1} \partial_{m}^{C}-(-1)^{p-1} \partial_{m-p}^{G} \xi_{m-p} f_{m}\right) \\
& =\delta_{p-1}\left((-1)^{p} \xi f\right)_{m},
\end{aligned}
$$

where we have assumed that $f_{m-1} \in \operatorname{ker}\left(d_{m-1}^{m-1-p}\right)$ and consequently $f_{m-1} \partial_{m}^{C}=0$. The above argument implies that, $[\phi f]=\left[\psi f+\delta_{p-1}(\xi f)\right]=[\psi f]$ and it naturally follows that $\iota_{\phi}=\iota_{\psi}$. We formalize this in the following Proposition: 
Proposition 5.4.5. There is a well-defined injective morphism $\iota: \prod_{n} H^{n}\left(C ; H_{n-p}(G)\right) \rightarrow$ $H^{p}(C ; G)$ given by $\iota=\iota_{\phi}$ for some choice of $\phi$. Moreover, this morphism is actually an isomorphism since it is an injective morphism between isomorphic groups.

Using the map just described, we write any $[f] \in H^{0}(C, G)$ as $[f]=\iota\left(\sum_{n}\left[f_{n}\right]\right)$ where $\left[f_{n}\right] \in H^{n}\left(C, H_{n}(G)\right)$ so we obtain

$$
P_{[f]}=P_{\sum_{n} \iota\left[f_{n}\right]}=\prod_{n} P_{\iota\left[f_{n}\right]}
$$

and based on the way $\iota$ was defined one sees that the operator $P_{\iota\left(f_{n}\right)}$ only acts non trivially on n-simplexes that intersect $f_{n}$. Therefore, operators such as $P_{\iota\left(f_{n}\right)}$ are analogous to the string operators used to define the ground states for the toric code.

Moreover, due to the fact that $\widehat{H^{0}(C ; G)}=\prod_{n} H^{n}\left(\widehat{C, H_{n}}(G)\right)$ any measurement operator can be decomposed in an similar fashion as

$$
Q_{r}=\sum_{n} Q_{\widehat{\iota^{-1}}}\left(r_{n}\right)
$$

where $r_{n} \in H^{n}\left(\widehat{C, H_{n}}(G)\right)$.

By direct calculation it follows that $\chi_{r}([f])=\prod_{n} \chi_{\iota^{-1}}\left(r_{n}\right)\left(\iota\left[f_{n}\right]\right)=\prod_{n} \chi_{r_{n}}\left(\left[f_{n}\right]\right)$ which implies

$$
Q_{r}\left|f_{G}\right\rangle=Q_{r} P_{[f]}|0\rangle=\prod_{n} \chi_{r_{n}}\left(\left[f_{n}\right]\right)\left|f_{G}\right\rangle
$$

Therefore the standard cohomology groups provide a source of quantum numbers since any ground state if of the form:

$$
\left|\left[f_{0}\right] \ldots\left[f_{n}\right]\right\rangle=\prod_{n} P_{\iota\left[f_{n}\right]}|0\rangle .
$$

In other words, we have shown that the ground state degeneracy underpins a convenient set of quantum numbers that label the states and that have been completely characterized. 


\section{Chapter 6}

\section{Final remarks}

We close this document first with a preliminary study of the excited states. We do this in a general context, taking advantage of the formalism introduced in the previous chapters. Although some progress have been obtained in this matter, we stress the fact that the excited states seem to require a case by case study, which we heuristically justify below.

Secondly, the last section of the chapter contains some final comments on the work presented. We emphasize some prospective work as well as some relations to other fields of research.

\subsection{Baby steps towards the study of the excited states}

In this section we outline, in an heuristic fashion, the basics about the nature of the grounds states for the formalism presented in Chapters 4 and 5. By reasons that will be apparent below, the analysis of the excited states seems to be lacking of an underlying classifying structure. Efforts are being made in order to settle this question by the research group. However, the latter situation remind us of the similar situations discussed for the generalizations of the QDM in Chapter 3, in which the braid groups and ribbon operators have not clear analogs describing the excited states for the generalized models presented there.

Let us begin by recalling that the ground state is basically characterized by the operator $\Pi_{0}$ of Definition 5.1.6. In fact, when acting over the operator $P_{[f]}$ defined in equation (5.2), that creates the ground state from the state $|0\rangle$, we have the following commutation 
relation:

$$
\Pi_{0} P_{[f]}=P_{[f]} \Pi_{0}, \quad \text { for } \quad[f] \in H^{0}(C, G) .
$$

The commutation relation just presented suggests that the study of the excited states can be done in a controlled way. The strategy will be to find expressions between the operators as characterizations of the states with higher energies. Let us consider the existence of some creation operator $0 \neq \vartheta: \mathcal{H}_{0} \rightarrow \mathcal{H} / \mathcal{H}_{0}$, such that, for any $|g\rangle \in \mathcal{H}_{0}$, we can construct the state $0 \neq\left|g^{\prime}\right\rangle:=\vartheta|g\rangle \in \mathcal{H} / \mathcal{H}_{0}$. Therefore, the following expression holds:

$$
\Pi_{0}\left|g^{\prime}\right\rangle=\Pi_{0}(\vartheta|g\rangle)=\Pi_{A^{0}} \Pi_{B^{0}} \vartheta|g\rangle=0 .
$$

We understand this expression as the obvious assertion that says that, when an excited state $\vartheta|g\rangle$ is projected into the ground state subspace through $\Pi_{0}$, it should give zero. Notice that this implies that there are two possible solutions for equation (6.1):

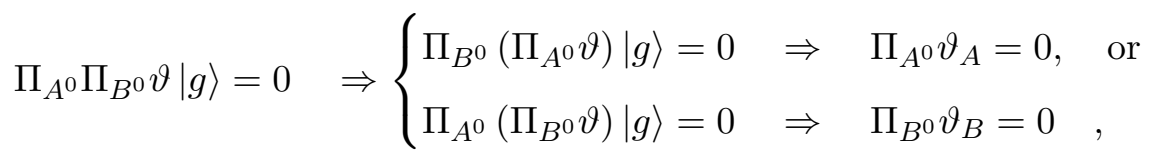

where we have used the commutation of the projector operators $\Pi_{A^{0}}$ and $\Pi_{B^{0}}$ (by Lemma 5.1.5 (i)) and, the frustration free property of our models. We have denoted the two types of operators by $\vartheta_{A}$ and $\vartheta_{B}$, depending on which projector operator they are canceling.

We can see that, by Lemma 5.1.5 (ii), we recognize these creation operators to be of the form:

$$
\begin{aligned}
& \vartheta_{A}:=\left.\Pi_{A^{s}}\right|_{s \neq 0}=\left.\prod_{n} \prod_{x \in K_{n}} A_{x}^{s_{n}(x)}\right|_{s_{n}(x) \neq 0} \quad \text { and } \\
& \vartheta_{B}:=\left.\Pi_{B^{h}}\right|_{h \neq 0}=\left.\prod_{n} \prod_{x \in K_{n}} B_{x}^{h_{n}(x)}\right|_{h_{n}(x) \neq 0},
\end{aligned}
$$

i.e. the generalized projector operators Defined in 5.1.1. This result was somehow already anticipated when discussing the properties of these generalized projectors in Chapter 5. We showed there that they form a complete set of projectors for different regions of the energy spectrum.

Furthermore, since we can write:

$$
\Pi_{A^{0}}=\prod_{n} \prod_{x \in K_{n}} A_{x}^{0_{n}(x)}, \quad \Pi_{B^{0}}=\prod_{n} \prod_{x \in K_{n}} B_{x}^{0_{n}(x)}
$$


and, due to the product form of the creation operators (6.2), we can write the equivalent expression

$$
\begin{aligned}
& \prod_{n} \prod_{x \in K_{n}}\left(A_{x}^{0_{n}(x)} A_{x}^{s_{n}(x)}\right)=0 \quad \text { and } \\
& \prod_{n} \prod_{x \in K_{n}}\left(B_{x}^{0_{n}(x)} B_{x}^{h_{n}(x)}\right)=0
\end{aligned}
$$

which, by virtue of Lemma 4.1.11, it implies that it suffices that at least one of the components $s_{n}(x)$ must be different different from zero and, analogously, it suffices that at least one of the components of $h_{n}(x)$ must be different from zero. Consequently, given the previous discussion, the simplest excited states are created by operators of the form:

$$
\begin{aligned}
& \vartheta_{A}^{s_{n}(x)}:=\left.A_{x}^{s_{n}(x)}\right|_{s_{n}(x) \neq 0_{n}(x)}=\left.\frac{1}{\left|G_{n+1}\right|} \sum_{g \in G_{n+1}} \chi_{s_{n}(x)}(g)\right|_{s_{n}(x) \neq 0_{n}(x)} A_{g x^{*}}, \\
& \vartheta_{B}^{h_{n}(x)}:=\left.B_{x}^{h_{n}(x)}\right|_{h_{n}(x) \neq 0_{n}(x)}=\left.\frac{1}{\left|G_{n-1}\right|} \sum_{r \in \hat{G}_{n-1}} \chi_{r}\left(h_{n}(x)\right)\right|_{h_{n}(x) \neq 0_{n}(x)} B_{r x_{*}} \quad,
\end{aligned}
$$

where $x \in\left\{K_{n}\right\}, h_{n}(x) \in G_{n-1}, s_{n}(x) \in \hat{G}_{n+1}$ and $g x^{*}$ and $r x_{*}$ are the local operators of Definition 4.1.8. We can see that there are basically creation operators for each generator $x \in\left\{K_{n}\right\}$ and for each map $r_{n}(x) x^{*} \in \operatorname{hom}(C, G)_{0}$ and $s_{n}(x) x_{*} \in \widehat{\operatorname{hom}}(C, G)_{0}$. In principle, it can be immediately seen that the latter is a big space to characterize and classify. In fact, up to this point, no classifying schemes has been devised to tackle this problem and, therefore the claim that the excitations must be basically studied case by case is justified.

Moreover, taking into account that the maps $\delta_{p}: \operatorname{hom}(C, G)_{p} \rightarrow \operatorname{hom}(C, G)_{p+1}$ and $\hat{\delta}_{p}: \widehat{\operatorname{hom}}(C, G)_{p} \rightarrow \widehat{\operatorname{hom}}(C, G)_{p-1}$ are injective, this is, im $\left(\delta_{p}\right) \subseteq \operatorname{hom}(C, G)_{p+1}$ and $\operatorname{im}\left(\hat{\delta}_{p}\right) \subseteq \widehat{\operatorname{hom}}(C, G)_{p-1}$, equations (6.4) can be cast in the following way:

Proposition 6.1.1. For every $g \in \hat{G}_{0}, h \in G_{0}$ and $x, y \in K_{n}$, such that $g y_{*} \in$ $\widehat{\operatorname{hom}}(C, G)_{0} / \operatorname{ker}\left(\hat{\delta}_{0}\right)$ and $h y_{*} \in \operatorname{hom}(C, G)_{0} / \operatorname{ker}\left(\delta_{0}\right)$. The following similarity transformations:

$$
\begin{aligned}
& Q_{g y_{*}} A_{x}^{0_{n}(x)} Q_{g y_{*}}^{-1}=A_{x}^{\left[\hat{\delta}_{0}\left(g y_{*}\right)\right]_{n}(x)} \quad \text { and } \\
& P_{h y^{*}} B_{x}^{0_{n}(x)} P_{h y^{*}}^{-1}=B_{x}^{\left[\delta_{0}\left(h y^{*}\right)\right]_{n}(x)} \text {, }
\end{aligned}
$$

hold. 
Proof. The first expression comes from the straight forward calculation:

$$
\begin{aligned}
Q_{g y_{*}} A_{x}^{0_{n}(x)} & =\frac{1}{\left|G_{n+1}\right|} \sum_{g \in G_{n+1}} Q_{g y_{*}} P_{\delta_{-1}\left(g x^{*}\right)} \\
& =\frac{1}{\left|G_{n+1}\right|} \sum_{g \in G_{n+1}} \chi_{g y_{*}}\left(\delta_{-1}\left(g x^{*}\right)\right) A_{g x^{*}} Q_{g y_{*}} \\
& =\frac{1}{\left|G_{n+1}\right|} \sum_{g \in G_{n+1}} \chi_{\sum_{i}\left[\hat{\delta}_{0}\left(g y_{*}\right)\right]_{i}(z) z_{*}}\left(g x^{*}\right) A_{g x^{*}} Q_{g y_{*}} \\
& =\frac{1}{\left|G_{n+1}\right|} \sum_{g \in G_{n+1}} \chi_{\left[\hat{\delta}_{0}\left(g y_{*}\right)\right]_{n}(x)}(g) A_{g x^{*}} Q_{g y_{*}} \\
& =A_{x}^{\left[\hat{\delta}_{0}\left(g y_{*}\right)\right]_{n}(x)} Q_{g y_{*},},
\end{aligned}
$$

from which the first expression is obtained. Analogously, the second expression follows:

$$
P_{h y^{*}} B_{x}^{0_{n}(x)}=B_{x}^{\left[\delta_{0}\left(h y^{*}\right)\right]_{n}(x)} P_{h y^{*}},
$$

from which the second expression is obtained.

It is clear that, by studying the operators of the form of Proposition 6.1.1, we could hope to systematize the analysis of the excited states. Therefore, let us try to understand the spaces that could be explored by such a method. In order to put things into more familiar grounds, we write the spaces of the latter proposition as

$$
\begin{aligned}
\operatorname{hom}(C, G)_{0} / \operatorname{ker}\left(\delta_{0}\right) & =\operatorname{im}\left(\delta_{-1}\right) / \operatorname{ker}\left(\delta_{0}\right) \oplus \operatorname{hom}(C, G)_{0} /\left(\operatorname{im}\left(\delta_{-1}\right) / \operatorname{ker}\left(\delta_{0}\right)\right) \\
& =H^{0(C, G)} \oplus \operatorname{hom}(C, G)_{0} / H^{0}(C, G),
\end{aligned}
$$

and

$$
\begin{aligned}
\widehat{\operatorname{hom}}(C, G)_{0} / \operatorname{ker}\left(\hat{\delta}_{0}\right) & =\operatorname{im}\left(\hat{\delta}_{1}\right) / \operatorname{ker}\left(\hat{\delta}_{0}\right) \oplus \widehat{\operatorname{hom}}(C, G)_{0} /\left(\operatorname{im}\left(\hat{\delta}_{1}\right) / \operatorname{ker}\left(\hat{\delta}_{0}\right)\right) \\
& =H^{-1}(C, G) \oplus \widehat{\operatorname{hom}}(C, G)_{0} / H^{-1}(C, G),
\end{aligned}
$$

where we have identified the quotients appearing in the previous relations by making repeated use of the second theorem of isomorphisms. It is easy to show the isomorphisms by means of the Figures 6.1, 6.2, 6.3 and 6.3. As a recall, the theorem asserts that the triangular diagrams seen there generate isomophisms between the two top spaces when the quotient group at the bottom has a trivial kernel. It can be checked that all of the diagrams have trivial kernels, thus, the isomorphism between the corresponding spaces is ensured. 


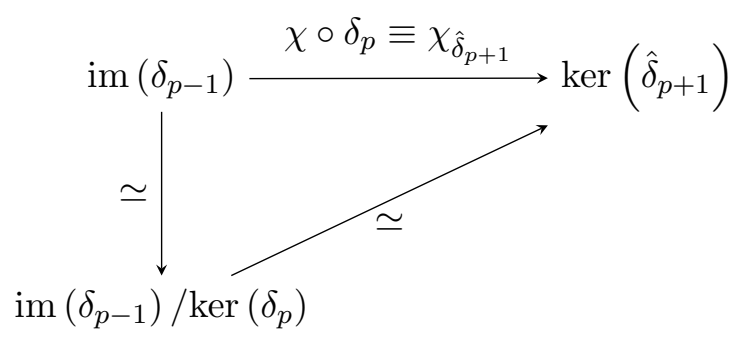

FiguRE $6.1: \quad \operatorname{im}\left(\delta_{p-1}\right) \cong \operatorname{ker}\left(\hat{\delta}_{p+1}\right)$

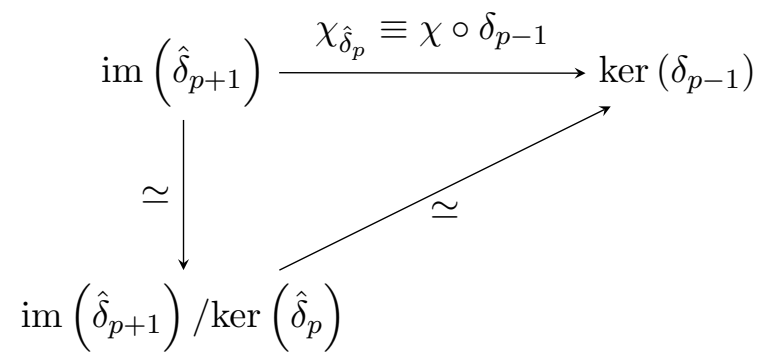

FiguRE $6.2: \operatorname{im}\left(\hat{\delta}_{p+1}\right) \cong \operatorname{ker}\left(\delta_{p-1}\right)$

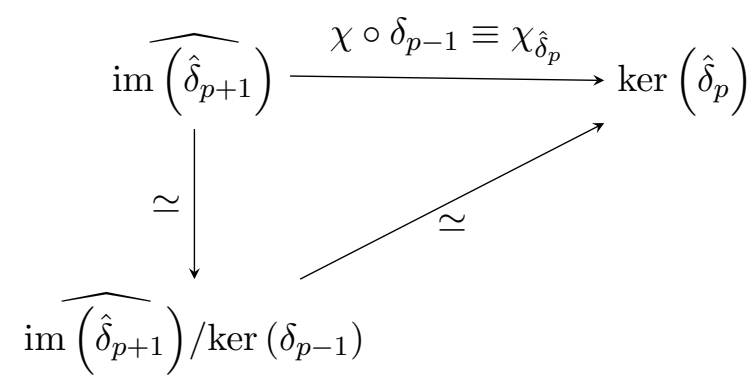

FIGURE 6.3: $\operatorname{im} \widehat{\left(\hat{\delta}_{p+1}\right)} \cong \operatorname{ker}\left(\hat{\delta}_{p}\right)$ and its dual $\operatorname{im}\left(\hat{\delta}_{p+1}\right) \cong \widehat{\operatorname{ker}\left(\hat{\delta}_{p}\right)}$

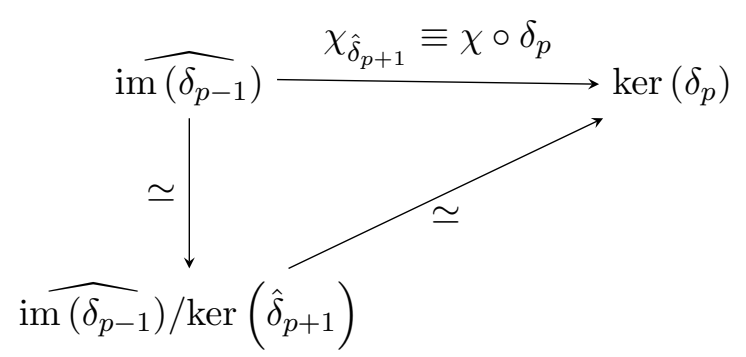

Figure 6.4: $\quad$ im $\widehat{\left(\delta_{p-1}\right)} \cong \operatorname{ker}\left(\delta_{p}\right)$ and its dual $\operatorname{im}\left(\delta_{p-1}\right) \cong \widehat{\operatorname{ker}\left(\delta_{p}\right)}$

In fact, by using the relations coming from Figures 6.1, 6.2, 6.3 and 6.3, we can easily show that:

$$
\widehat{H^{p}(C, G)}=\frac{\widehat{\operatorname{ker}\left(\delta_{p}\right)}}{\operatorname{im(\delta _{p-1})}}=\frac{\operatorname{im} \widehat{\left(\hat{\delta}_{p+2}\right)}}{\operatorname{ker}\left(\hat{\delta}_{p+1}\right)}=\frac{\operatorname{ker}\left(\hat{\delta}_{p+1}\right)}{\operatorname{im}\left(\hat{\delta}_{p+2}\right)} .
$$


We can see that by the decompositions (6.5) and (6.6), the exploration of the excited states through the the similarity transformations 6.1.1 gets short and it is not suitable to reproduce the entire excited space spectrum. Further analysis in this line of thought will be done in forthcoming works as well as a more exhaustive study of different particularizations of the general formalism.

A few comments are in order. As it became clear throughout the previous discussion, the generalized projector operators of definition 5.1.1 describe subspaces of $\mathcal{H}$ that correspond to the excited states of the model when the parameters in Definition 5.1.1 are non trivial. The statistics of the excitations can then be obtained from the commutation relations of the generalized projectors. We also expect these operators to play a fundamental role when studying the bulk-boundary correspondence in the case when the $C$ complex comes from a manifold with boundaries.

\subsection{Summing up}

In this work we have generalized the structures of lattice gauge theories to arbitrary dimensions. We have done this by basing the formalism on algebro-topological methods. The generalization obtained sacrifices mathematical structure for range, since the models work for abelian higher gauge. To be concrete, we have shown that the cohomology $H^{p}(C, G)$ C.2.6 [52], is a natural structure for the study of the quantum features of the class of models introduced in Chapter 4. These models encompass known models already available in the literature like the Abelian Quantum Double and its generalizations. Also, under certain restrictions for the action maps of $n$-groups, the formalism presented describe theories with higher gauge transformations of abelian groups of any order, all at once.

The most important feature of the formalism is that it has been used to explicitly show how the topology of all these models describes the ground state degeneracy. All of this in a unique theoretical framework. Particularly, it was shown in Chapter 5 that the ground state degeneracy of is characterized by the 0-th cohomology group $H^{0}(C, G)$. The expression derived allows to explicitly and unambigously obtain the GSD of all of the models discussed, if the homology of the chains $C$ and $G$ are known. Furthermore, $C$ is usually associated to a simplicial complex, hence its topology is already known for a large set of compact manifolds. If $G$ is composed by a graded group, its homology is computable and the 0 -th cohomology group $H^{0}(C, G)$ can be obtained by means of the generalized coefficient theorem. 
In the same line, through Section 5.4, it has been proven that the isomorphism $\left|H^{0}(C, G)\right| \cong$ $\prod_{n}\left|H^{n}\left(C, H_{n}(G)\right)\right|$ underpins a suitable way to completely characterize the basis of the ground state space $\mathcal{H}_{0}$ of these models in terms of elements of $\prod_{n}\left|H^{n}\left(C, H_{n}(G)\right)\right|$. Moreover, also in 5, we have shown an explicit isomorphism that can be understood as a complete set of quantum numbers for these systems. Unfortunately, even though this characterization is complete, it comes at the stake of a clear geometrical interpretation of the ground states, since its elements are now in a one-to-one correspondence with the elements of $\prod_{n}\left|H^{n}\left(C, H_{n}(G)\right)\right|$ which, in turn, mix the geometrical content (associated to the $C$ complex) with that of the group content (associated to the $G$ complex).

In short, if the chains $C$ and $G$ are as described above, this formalism blunders geometry and group structure into a single, more powerful structure, that completely characterizes the ground state. However, due to the same reason, the physical interpretation gets obscure and the ground are in general described by intrincate configurations. We believe that the gain in analiticity overpasses the lack of neat physical interpretation and new tools can be developed to also gain further physical insight of these models in the future.

\subsection{Future work}

We close by presenting several possible work related to the content of this thesis:

(i) As it was commented at the end of Chapter 5, taking the $C$ complex to be any free finitely generated complex will produce a new class of topological models that are yet to be explored, and for which the formalism presented can be immediately applied without any modification.

(ii) Another promising way to study these models was already outlined at the introduction. As presented in [79], quantum error correction codes follow relatively easy from a Homological perspective, which in this case is naturally provided by the formalism exposed. Since, also in the present work, the GSD has been completely characterized, the number of logical qubits that can be stored in the code is known. Moreover, the set of logical operators gets also completely determined by the quantum numbers given by Theorem 5.2.3. The duality between the operators that map between different ground state and their corresponding measuring operators is explicit in this formalism as it is shown in Section 5.4.1. This algebraic duality also determines the geometric duality between operators since ground-state mapping and measuring operators have different dimensionality (strings, membranes, etc..). 
(iii) In a related vein, the existence of good quantum correcting codes for these systems, as defined in the pioneering works [88-90], is also an open question that will be pursued in following works.

(iv) In Section 5.3 we show how our models reproduce some well known topological models. The relation between the models we obtain in this work and symmetry protected phases is not fully understood. However, in [20] SPT phases with higher global symmetry in $d$ dimensions are shown to have a duality relation with intrinsic topologically ordered phases with local symmetry via a gauging map that is precisely defined in [21] for the usual SPT phases. Thus, the models we introduce in this paper should have a duality relation with higher symmetry SPT phases.

(v) An important remark is that, even though for the examples here presented we considered the chain complex $C$ as coming from closed manifolds, the formalism also accounts for chain complexes of compact manifolds with boundaries and more general chain complexes not related to geometry. For all those cases the GSD formula of Theorem 5.2.3 is still valid. This gives the current formalism an exploration flexibility that will be studied in the future.

(vi) Since the models presented in this piece of work are written in the language of Homology, they are naturally interpreted as the higher dimensional versions of the so called homological quantum error correction codes [85-87]. Furthermore, the closed formula we have obtained for the GSD of the model determines both the coding space and the labels of the logical operators.

(vii) The study of the excited states is also an open question in the case of these models. It is yet to be seen if the cohomology with coefficients in a chain complex have a greater role to play in the description of them. 


\section{Appendix A}

\section{Basic Differential geometry and Simplicial Decomposition}

This is a brief cover of the main mathematical for the 1-gauge continuous theory. Of course, this is by no means a complete account on the topics. The interested reader is invited to consult the bibliography for further information, particularly [54, 92, 93] for non mathematicians.

\section{A.1 Basics of general topology}

In this section we present the basics of general topology as applied to topological manifolds. This structure is usually taken as the geometric support of the models presented in this work.

Definition A.1.1 (Topological Space). Let $X$ be any set and $Y=\left\{X_{\alpha}\right\}$ denote a collection, finite or infinite of subsets of $X$. Then $X$ and $Y$ form a topological space provided the $X_{\alpha}$ and $Y$ satisfy:

1. $\emptyset \in Y, X \in Y$.

2. Any finite or infinite subcollection $\left\{Z_{\alpha}\right\}$ of the $X_{\alpha}$ has the property that $\bigcup_{\alpha} Z_{\alpha}$.

3. Any finite subcollection $\left\{Z_{\alpha_{1}}, \cdots, Z_{\alpha_{n}}\right\}$ of the $X_{\alpha}$ has the property that $\bigcap Z_{\alpha_{i}} \in$ $Y$. 
the set $X$ is then called a topological space and the $X_{\alpha}$ are called open sets. The choice of $Y$ satisfying (A.1) is said to give a topology to $X$.

The following properties are important when determining the possibility of connecting different manifolds through well behaved maps

Definition A.1.2 (Hausdorff Spaces). A Hausdorff Space is a topological space for which if any two distinct points $x, y \in X$ there exists a pair of open sets $O_{x}$ and $O_{y}$, respectively, such that $O_{x} \bigcap O_{y}=\emptyset$. See figure (A.1)

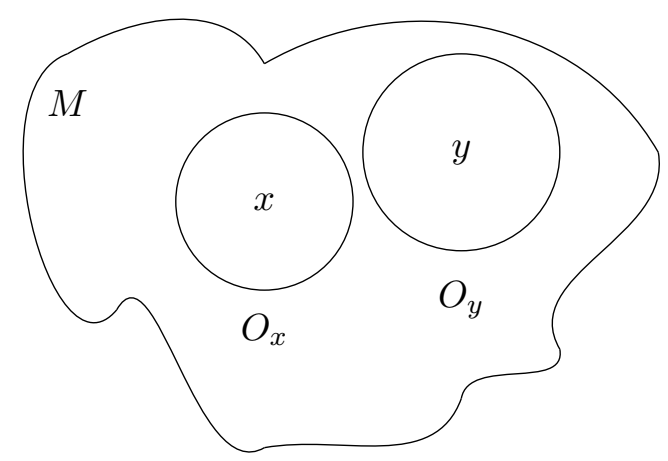

Figure A.1: Hausdorff axiom of separation, the open neighborhoods of $x\left(O_{x}\right)$ and $y$ $\left(O_{y}\right)$ do not intersect each other

Definition A.1.3 (Continuity). A function $f: X \rightarrow Y$, where $X$ and $Y$ are two topological spaces, are said to be continuous if the inverse image of an open set in $Y$ is an open set in $X$.

The latter definitions are generalizations of the analogous concepts in the case of the regular one-variable calculus. In fact, it is easy to show that these definitions are equivalent to the separation properties and the " delta" definition of Cauchy. Following is more structure that will allow to define measures and integration over a manifold.

Definition A.1.4 (Open Coverings). Let us consider the set $U$. Given a family of open sets $\left\{F_{\alpha}\right\}=F$, then $F$ is an open cover of $U$ if $U \subset \bigcup_{\alpha} F_{\alpha}$.

Definition A.1.5 (Compactness). Consider the set $U$ and all its open coverings. The set $U$ is compact if for every open covering $\left\{F_{\alpha}\right\}$ with $U \subset \bigcup_{\alpha} F_{\alpha}$ there always exist a finite subcovering $\left\{F_{1}, \cdots, F_{n}\right\}$ of $U$ with $U \subset \bigcup_{i} F_{i}$ with $i=1, \cdots, n$.

The most suitable way to study two manifolds is by comparing it to more familiar ones, this is usually done by finding the following maps: 
Definition A.1.6 (Homeomorphism). Let us take two different topological spaces $T_{1}$, $T_{2}$ and a map $\alpha: T_{1} \rightarrow T_{2}$ is said to be a homeomorphism if it is both continuous and has an inverse which is also continuous.

The kind of manifolds we are interested are those that can be decomposed, specially those that can be analyzed through simplicial decomposition, although some other compositions can be used. We focus on smooth manifolds, mainly because many differential and algebraic properties (like the chain property of the exterior derivative) have their analogs in the discrete, which will be our case.

Definition A.1.7 (Charts and atlas). Given a topological space $M$, a chart (or local coordinate map) is a pair, $(U, \phi)$, where $U$ is an open subset of $M$ and $\phi: U \rightarrow \Omega$ is a homeomorphism onto an open subset, $\Omega=\phi(U)$, of $\mathbb{R}^{n_{\phi}}$ (for some $n_{\phi} \geq 1$ ). A complete set of local charts $\left\{\left(M_{\alpha}, \phi_{\alpha}\right)\right\}$ is called an atlas.

These local coordinates allows us to write things around a point of the manifold, however the dynamics of any system is usually understood in such a background by differential relations. Hence, the following type of manifolds are a very natural background for physical modeling:

Definition A.1.8 (Differentiable Manifold). A set $M$ is a Differentiable Manifold if:

(i) $M$ is a topological space.

(ii) $M$ is provided with a family of charts $\left\{M_{\alpha}, \phi_{\alpha}\right\}$.

(iii) The $M_{\alpha}$ are a family of open sets which cover $M=\bigcup_{\alpha} M_{\alpha}=M$. The $\phi_{\alpha}$ are homeomorphisms from $M_{\alpha}$ to an open subset $O_{\alpha}$ of $\mathbb{R}^{n}, \phi_{\alpha}: M_{\alpha} \rightarrow \phi_{\alpha}$.

(iv) Given $M_{\alpha}, M_{\beta}$ such that $M_{\alpha} \cap M_{\beta} \neq \emptyset$, the map $\phi_{\beta} \circ \phi_{\alpha}^{-1}$ from the subset $\phi_{\alpha}\left(M_{\alpha} \bigcap M_{\beta}\right)$ of $\mathbb{R}^{n}$ to the subset $\phi_{\beta}\left(M_{\alpha} \cap M_{\beta}\right)$ of $\mathbb{R}^{n}$ is infinitely differentiable $\left(C^{\infty}\right)$.

See figure (A.2).

In other words, a differential manifold locally looks like a $\mathbb{R}^{n}$. However, other properties can be non trivial, for instance, the notion of orientability, which in the case of $\mathbb{R}^{n}$. We refine this concept in the following definition:

Definition A.1.9 (Orientability). Given a manifold $M$ with atlas $\left\{\left(M_{\alpha}, \phi_{\alpha}\right)\right\} . M$ is orientable if $\operatorname{det}\left(\phi_{\alpha} \circ \phi_{\beta}^{-1}\right)>0$ for all $M_{\alpha}, M_{\beta}$ such that $M_{\alpha} \cap M_{\beta} \neq \emptyset$. See figures (A.3) and (A.4). 
$\mathrm{h} !$

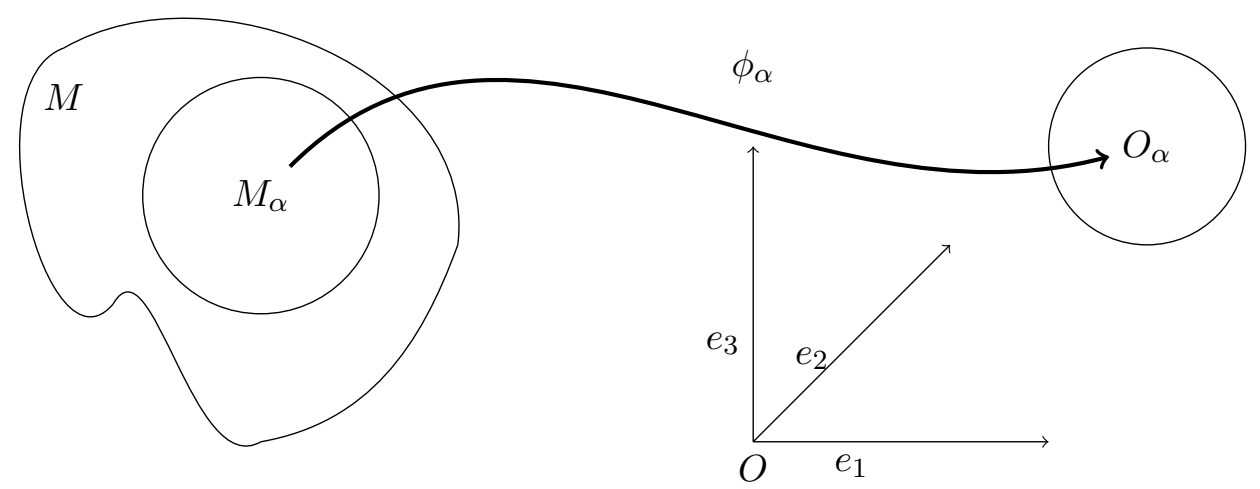

Figure A.2: Open cover $M_{\alpha}$ go to an open subset $O_{\alpha}$ through the homeomorphic $\operatorname{map} \phi_{\alpha}$

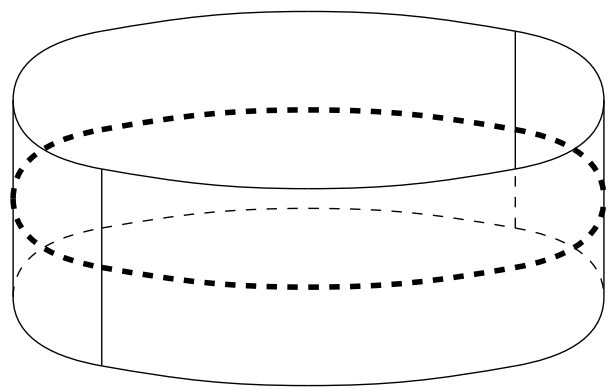

Figure A.3: An orientable manifold (strip surface)

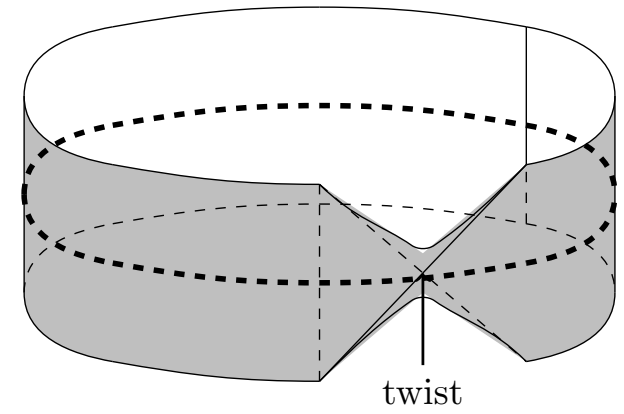

Figure A.4: A non orientable manifold (a Möbius strip)

The previous notion of orientability can be extended to the finite discrete case quite straightforwardly, by requiring that each one of the components of a discrete manifold is orientable. In what follows, we will be using Einstein convention for the sum over repeated indexes and every manifold is a finite dimensional manifold, unless stated.

The main goal is to build a natural vector space in each point by realizing that such a space can be constructed by the tangent structure to each point of a differentiable manifold. We start by considering the point $p \in M$, which is compact and orientable, according to Definitions A.1.5 and A.1.9, respectively. Let us consider a curve parametrized by 
$t$ to be $p(t)$. This one can be written in local coordinates $x^{i}$ with $i=1, \cdots, n$. Hence, we can write the tangent vector to the curve $p(t)$ as $\frac{d x^{i}(p(t))}{d t}$ with $i=1, \cdots, n$. It is clear that this tangent vector to a point is not unique, therefore the solution is to take the following equivalent class:

Definition A.1.10 (Equivalence class of tangent vectors). Given a differentiable manifold $M$ of dimension $n$, for any $p \in M$, two $C^{1}$-curves (continuously differentiable), $\left.\gamma_{1}:\right] \epsilon_{1}, \epsilon_{1}\left[\rightarrow M\right.$ and $\left.\gamma_{2}:\right] \epsilon_{2}, \epsilon_{2}\left[\rightarrow M\right.$, through $p$ (i.e., $\left.\gamma_{1}(0)=\gamma_{2}(0)=p\right)$ are equivalent iff there is some chart, $(U, \phi)$, at $p$ so that

$$
\left(\phi \circ \gamma_{1}\right)^{\prime}(0)=\left(\phi \circ \gamma_{2}\right)^{\prime}(0)
$$

We are now in position to define the natural vector structure arising from a differentiable manifold. It is worth noticing that there are other equivalent constructions stressing some other mathematical properties in passing. However, for our current summary, the previous material suffices to define:

Definition A.1.11 (Tangent Manifold). Given a differentiable $n$-manifold $M$. For any $p \in M$, a tangent vector to $M$ at $p$ is any equivalence class of $C^{1}$-curves through $p$ on $M$, modulo the equivalence relation of Definition A.1.10. The set of all tangent vectors at $p$ is denoted by $T_{p}(M)$.

It is easy to show that $T_{p}(M)$ is indeed a vector space. The question of defining its base is a bit more technical. We embark on its formal definition by means of the germs, that we pass to define in what follows:

Definition A.1.12 (Germs). Given any differential $n$-manifold $M$. For any $p \in M$, a locally defined function at $p$ is a pair, $(U, f)$, where $U$ is an open subset of $M$ containing $p$ and $f$ is a function defined on $U$. Two locally defined functions, $(U, f)$ and $(V, g)$, at $p$ are equivalent iff there is some open subset, $W \subseteq U \cap V$, containing $p$ so that

$$
f \rightarrow W=g \rightarrow W
$$

The equivalence class of a locally defined function at $p$, denoted $[f]$ or $\mathbf{f}$, is called a germ at $p$.

It is possible to define addition of germs, multiplication of a germ by a scalar and multiplication of germs, in the obvious way which turn germs into a ring, algebraically speaking. The ring of germs of $C^{k}$-functions ( $k$ differentiable functions) at $p$ is denoted by $\mathcal{O}_{M, p}^{(k)}$. We say that $\mathbf{f}$ is a stationary germ iff $\left(f \circ \phi^{-1}\right)^{\prime}(\phi(p))=0$, for some chart $(U, \phi)$ at $p$ and some function $f$ belonging to the germ $\mathbf{f}$. Furthermore, it can be proven 
that the $C^{k}$-stationary germs form a subring of $\mathcal{O}_{M, p}^{(k)}$ (but not an ideal), that we will denote by $\mathcal{S}_{M, p}^{(k)}$. Un der this framework, the quotient group $\mathcal{O}_{M, p}^{(k)} / \mathcal{S}_{M, p}^{(k)}$ is isomorphic to the tangent space $T_{p}(M)$ and we can state the following proposition:

Proposition A.1.13 (Tangent space basis). Given any $C^{k}$-manifold $M$, of dimension $n$, with $k \geq 1$, for any $p \in M$ and any chart $(U, \phi)$ at $p$, the $n$ functions, $\left(\frac{\partial}{\partial x_{1}}\right)_{p}, \cdot,\left(\frac{\partial}{\partial x_{n}}\right)_{p}$ defined on $\mathcal{O}_{M, p}^{(k)}$ by

$$
\left(\frac{\partial}{\partial x_{i}}\right)_{p} f:=\left.\frac{\partial f \circ \phi^{-1}}{\partial X_{i}}\right|_{\phi(p)}, \quad 1 \geq i \geq n
$$

are linear forms that vanish on $\mathcal{S}_{M, p}^{(k)}$. Where $\left.\left(\frac{\partial g}{\partial X_{i}}\right)\right|_{y}$ denotes the partial derivative of a function $g: \mathbb{R}^{n} \rightarrow \mathbb{R}$ with respect to the ith coordinate, evaluated at $y$. Every linear form $L$ on $\mathcal{O}_{M, p}^{(k)}$ that vanishes on $\mathcal{S}_{M, p}^{(k)}$ can be expressed in a unique way as

$$
L=\left.\sum_{i=1}^{n} \lambda_{i}\left(\frac{\partial}{\partial x_{i}}\right)\right|_{p}
$$

where $\lambda_{i} \in \mathbb{R}$. Therefore, the

$$
\left.\left(\frac{\partial}{\partial x_{i}}\right)\right|_{p}, \quad 1 \geq i \geq n
$$

form a basis of the vector space of linear forms on $\mathcal{O}_{M, p}^{(k)}$ that vanish on $\mathcal{S}_{M, p}^{(k)}$.

Since this is a basic result of differential geometry, it will be just stated here. For those interested readers we refer to [94, 95]. We now turn into the construction of the dual of the tangent space. It is important to stress that many of these structures are mirrored in the discrete case and, hence, it is appropriate to be familiar with them at this stage. We recall the reader that, informally speaking, a derivation $d$ is an operator that fulfills the Leibniz rule $d(a b)=(d a) b+a(d b)$ for any two elements $a, b$ of a ring space. With this, the construction procedure of the dual space is similar to the one sketched above:

Definition A.1.14 (Cotangent space). Given any $C^{k}$-manifold $M$, of dimension $n$, with $k \geq 1$, for any $p \in M$, the tangent space at $p$, denoted by $T_{p}(M)$, is the space of linear derivations on $\mathcal{O}_{M, p}^{(k)}$ that vanish on $\mathcal{S}_{M, p}^{(k)}$. Thus, $T_{p}(M)$ can be identified with $\left(\mathcal{O}_{M, p}^{(k)} / \mathcal{S}_{M, p}^{(k)}\right)^{*}$. The space $\mathcal{O}_{M, p}^{(k)} / \mathcal{S}_{M, p}^{(k)}$ is called cotangent space at $p$; it is isomorphic to the dual $T_{p}^{*}(M)$ of $T_{p}(M)$. 
If we denote by $\pi_{i}$ the canonical projector of the $i$-th local coordinate, so we define $x_{i}:=\pi_{i} \circ \phi$, we observe that the simple calculation

$$
\left(\frac{\partial}{\partial x_{i}}\right)_{p} x_{j}=\delta_{j}^{i},
$$

defines an isomorphism that gives a representation of the basis. This is, the images of $x_{1}, \cdots, x_{n}$ in $\mathcal{O}_{M, p}^{(k)} / \mathcal{S}_{M, p}^{(k)}$ are dual of the basis $\left(\frac{\partial}{\partial x_{1}}\right)_{p}, \cdot,\left(\frac{\partial}{\partial x_{n}}\right)_{p}$ of $T_{p}(M)$. Hence, given any $C^{k}$-function $f$ on $M$, we denote the image of $f$ in $T_{p}^{*}(M)$ by $d f_{p}$ that we recognize as being the differential of $f$ at $p$. Using the isomorphism between $\mathcal{O}_{M, p}^{(k)} / \mathcal{S}_{M, p}^{(k)}$ and $\left(\mathcal{O}_{M, p}^{(k)} / \mathcal{S}_{M, p}^{(k)}\right)^{* *}$ described above, $d f_{p}$ corresponds to the linear map in $T_{p}^{*}(M)$ defined by $d f_{p}(v)=v(\mathbf{f})$, for all $v \in T_{p}(M)$. Furthermore, with this notation, we see that $\left(d x_{1}\right)_{p}, \cdots,\left(d x_{n}\right)_{p}$ is a basis of $T_{p}^{*}(M)$, dual to the basis $\left(\frac{\partial}{\partial x_{1}}\right)_{p}, \cdot,\left(\frac{\partial}{\partial x_{n}}\right)_{p}$ of $T_{p}(M)$. What is left is to extend these local definitions at a point $p \in M$ to the entirety of the points of the manifold $M$. This is done in a very canonical way, as follows:

Definition A.1.15 (Tangent bundle). We define $T(M)$ the tangent bundle, to be the disjoint union of the tangent spaces $T_{p}(M)$ for all $p \in M$, such that there is natural projection

$$
\pi: T(M) \rightarrow M, \quad \text { where } \quad \pi(v)=p \quad \text { if } v \in T_{p}(M)
$$

We still have to give $T(M)$ and to define a $C^{k-1}$-atlas. For every chart, $(U, \phi)$ of $M$ (with $U$ open in $M$ ) we define the function $\tilde{\varphi}: \pi^{-1}(U) \rightarrow \mathbb{R}^{2 n}$ by

$$
\tilde{\varphi}(v)=\left(\phi \circ \pi(v), \theta_{U, \phi, \pi(v)}^{-1}\right)
$$

where $v \in \pi^{-1}(U)$ and $\theta_{U, \phi, \pi(v)}: \mathbb{R}^{n} \rightarrow T_{p}(M)$ is the natural isomorphism defined by $\theta_{U, \phi, \pi(v)}: \mapsto[(U, \phi, u)]$ for all $u \in \mathbb{R}^{n}$. It is obvious that $\tilde{\varphi}$ is a bijection between $\pi^{-1}(U)$ and $\phi(U) \times \mathbb{R}^{n}$, an open subset of $\mathbb{R}^{2 n}$. We give $T(M)$ the weakest topology that makes all the $\tilde{\varphi}$ continuous, i.e. we take the collection of subsets of the form $\tilde{\varphi}^{-1}(W)$, where $W$ is any open subset of $\phi(U) \times \mathbb{R}^{n}$, as a basis of the topology of $T(M)$. Furthermore, it can be checked that $T(M)$ is Hausdorff and second countable in this topology.

Analogously, we can do the same with the cotangent spaces:

Definition A.1.16 (Cotangent bundle). We define $T^{*}(M)$ the cotangent bundle, to be the disjoint union of the cotangent spaces $T_{p}^{*}(M)$ for all $p \in M$, such that there is natural projection

$$
\pi: T^{*}(M) \rightarrow M, \quad \text { where } \quad \pi\left(v^{*}\right)=p \quad \text { if } v^{*} \in T_{p}^{*}(M)
$$


The topology is constructed in a similar fashion. For any chart, $(U, \phi)$ on $M$ we define the function $\tilde{\varphi}: \pi^{-1}(U) \rightarrow \mathbb{R}^{2 n}$ by

$$
\tilde{\varphi}(\tau)=\left(\phi \circ \pi(\tau), \tau\left(\left(\frac{\partial}{\partial x_{1}}\right)_{\pi(\tau)}\right), \cdots, \tau\left(\left(\frac{\partial}{\partial x_{n}}\right)_{\pi(\tau)}\right)\right),
$$

where $\tau \in \pi^{-1}(U)$ and $\left(\frac{\partial}{\partial x_{1}}\right)_{p}$ are the basis of $T_{p}(M)$ associated with the chart $(U, \phi)$. It follows that you can make $T^{*}(M)$ into a $C^{k-1}$-manifold of dimension $2 n$. Notice that for every chart $(U, \phi)$ on $M$ there is a bijection $\tau_{U}: \pi^{-1}(U) \rightarrow U \times \mathbb{R}^{n}$, given by $\tau_{U}(v)=\left(\pi(v), \theta_{U m \phi, \pi(v)}^{-1}\right)$. Clearly, $\operatorname{pr}_{1} \circ \tau_{U}=\pi$ on $\pi^{-1}(U)$. Thus, over $U$, the bundle $T(M)$ looks like the product $U \times \mathbb{R}^{n}$. We say that $T(M)$ is locally trivial over $U$ and $\tau_{U}$ is called a trivializing map.

Over the structures already discussed, for any $p \in M$, the vector space $\pi^{-1}(p)=T_{p}(M)$ is called the fibre above $p$ and, respectively, For any $p \in M$, the vector space $\pi^{-1}(p)=$ $T_{p}(M)$ is called the fibre above $p$. This construction is of the uttermost importance for the definition of a continuous 1-gauge theory, as it is briefly explained in Chapter 2. In there, we discuss that the fibre can be associated to elements of a Lie group $G$ and the ideas arising from there will be the basis of the discrete gauge theories we will further discuss.

We have seen that a differentiable manifold is, loosely speaking, a well behaved space. The following section will describe how to discretize this geometrical space, which will ultimately be the geometric support of the theories described in this document.

\section{A.2 Simplices and Simplicial Complexes}

Let us suppose that the differeable manifold $M$ of the previous section is homeomorphic to some polyhedron $K$. We can then identify a set of points $\left\{x_{i}\right\} \in K, i \in I$ where $I$ is a set of labels, such that they coincide in one-to-one correspondence with the vertices of the polyhedron. We call this set of points independent if they are linearly independent. We also call this set of points ordered if they have been completely identified by their labels. Hence, in this context the following objects can be constructed

Definition A.2.1. An oriented $p$-simplex, written $\sigma^{p}$ for $p>1$ is a set of points $\left\{x_{i}\right\} \in$ $K, i \in I$ such that

$$
\sigma^{p}=\left\{x=\sum_{i=1}^{p+1} \lambda_{i} x_{i} \quad \lambda_{i} \geq 0 \quad, \quad \sum_{i=1}^{p+1} \lambda_{i}=1\right\}
$$

where $x_{1}, \ldots, x_{p+1}$ are independent and ordered. 
From now on an ordered simplex will be written $\sigma^{p}=\left[x_{1}, \ldots, x_{p+1}\right]$, representing the class of even permutations $+\sigma^{p}$ of the chosen ordering, while the odd permutation are denoted $-\sigma^{p}$. To have a geometrical grasp of this decomposition, let us consider $\mathbb{R}^{3}$, the kind of simplices that can be constructed are shown in figure A.5.

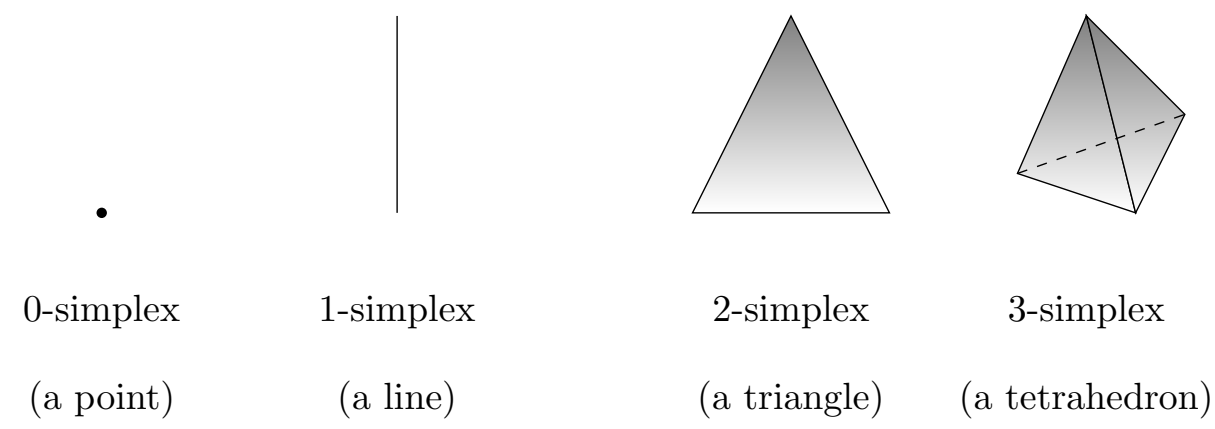

Figure A.5: Simplices in a $\mathbb{R}^{3}$ space

In order to maintain as much as possible the mathematical structure of the continuous case described in the previous section, we define the following:

Definition A.2.2 (Simplicial boundary map). Let $X^{n} \in K$ be an $n$-simplex with set of vertices $\left\{x_{0}, x_{1}, \cdots, x_{n}\right\}$. The boundary of $K^{n}$, denoted $\partial K^{n}$, is a set of simplices obtained from proper and non-empty subsets of $\left\{x_{0}, x_{1}, \cdots, x_{n}\right\}$.

And also the type of simplicial complexes we will mainly deal with:

Definition A.2.3 (Oriented simplicial complex). A simplicial complex $\mathbf{K}$ is the set of all $p$-simplexes that can be constructed from the set of points $x \in K$. If those simplexes have been assigned an orientation then this structure is known as an oriented simplicial complex.

In fact, in an oriented simplicial complex, we can define the boundary map $\partial:\left\{X^{n}\right\} \rightarrow$ $\left\{X^{n-1}\right\}$ in such a way that preserves the orientation. In order to be concrete let us take a 2-simplex (or 2-chain) $\sigma^{2}=\left[x_{0}, x_{1}, x_{2}\right] \in K$, owing to figure A.5, in an Euclidean oriented simplicial complex this will correspond to a triangle with ordered vertices labelled as $x_{0}, x_{1}$ and $x_{2}$, as it can be seen in figure A.6. Let us and apply the boundary operator on it

$$
\partial \sigma^{2}=\partial\left[x_{0}, x_{1}, x_{2}\right]=\left[x_{1}, x_{2}\right]-\left[x_{0}, x_{2}\right]+\left[x_{1}, x_{2}\right] .
$$

Note that the boundary operator gives a linear combination of 1-simplices. Furthermore, this construction is coherent with the way in which two 1-simplices (oriented lines) 

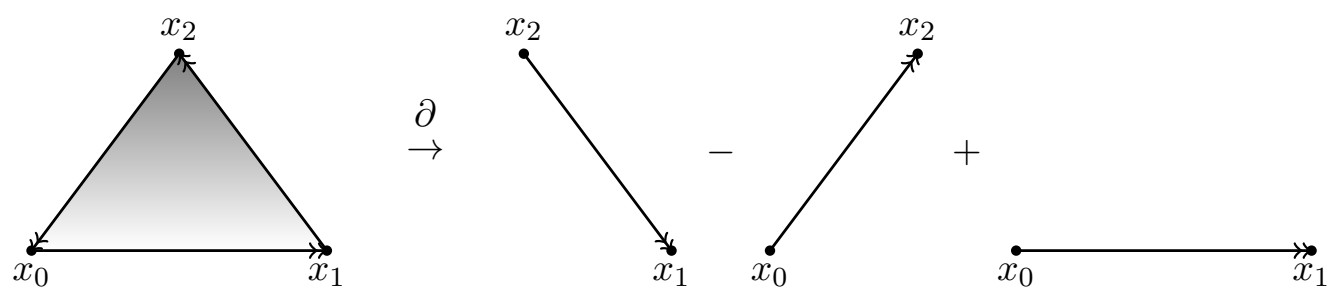

FiguRE A.6: Schematic representation of a boundary operator acting over a 2-simplex

are summed together, a fact that can be easily proven by doing the straight forward calculation. The previous operation can be easily generalized to simplices of higher order to a $\sigma^{p} \in K$ to be

$$
\partial \sigma^{p}=\partial\left[x_{0}, x_{1}, \cdots, x_{p}\right]:=\sum_{i=0}^{p}\left(-1^{i}\right)\left[x_{0}, x_{1}, \cdots, \hat{x}_{i}, \cdots, x_{p}\right]
$$

where the symbol $\hat{x}_{i}$ represents absence of that element from the list of the ordered simplex of order $p$.

In order to keep restricting the type of spaces we will dealing with, we characterize even further the type of ordered simplicial complexes we are interested in this work:

Definition A.2.4 (Euclidean complex). Let $K$ be a finite set of simplices that are subsets of a fixed $\mathbb{R}^{m}$. Assume that the intersection of any two members of $\mathbf{K}$ is empty. Require, furthermore, that for every simplex $x \in K$, all elements of its boundary $\partial x$ are also elements of $K$. Then $K$ is called a (finite) Euclidean complex.

The importance of the later specification is that we are willingly avoiding certain patological manifolds in our study. It will be understood that we will be referring only to Euclidean complexes from now on. However, the notion of a $p$-simplex is general enough to allow the study more general manifolds. We are now in position to study manifolds by means of simplicial complexes. This is done by the aim of the following definition

Definition A.2.5 (Triangulable manifold). A manifold is is said to be triangulable if there is an homeomorphism $\mathbf{K} \rightarrow M$ ( $M$ usually a $\left.V \subset \mathbb{R}^{n}\right)$.

which foreshadow the importance of this decomposition. We can make this connection more formal by means of the following Lemma:

Lemma A.2.6 (Geometric realization of simplicial complex). Any simplicial complex has a geometric realization. 
Proof. Let $K$ be a simplicial complex. Consider the 1-element sets contained in $K$. Denote by $e_{1}, e_{2}, \cdots, e_{n}$ the elements of these sets. Then any element of $K$ is the union of some of these elements. It is clear that by identifying each vertex of these elements with some coordinate of $\mathbb{R}^{n}$ we have a geometric realization of an Euclidean complex, i.e. an underlying polyhedron that yields a geometric realization of $K$.

A triangulation of a 2-manifold of genus 1 is shown in figure A.7

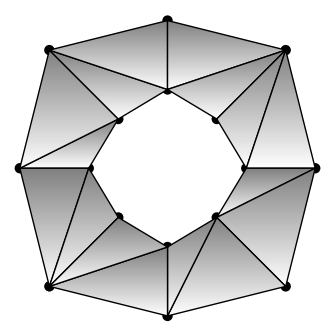

Figure A.7: Triangulation of a closed annulus. It has 16 0-cells, 32 1-cells and 16 2-cells

Hence, a triangulation of a manifold is a proper construction for where to use the simplicial decomposition of manifolds just described, since it will carry all the basic information featured in a continuous 1-gauge theory. Moreover, the topological features are also contained in this formulation, as the following discussion implies.

Definition A.2.7 (Refinement). Let $M$ be a manifold. Let $K, L$ be simplicial complexes with triangulations $\tau_{K}: \mathbf{K} \rightarrow M$ and $\tau_{L}: \mathbf{L} \rightarrow M$ of the manifold $M$. If $\sigma: \tau_{K}^{-1} \circ \tau_{L}$ : $\mathbf{K} \rightarrow \mathbf{L}$ maps simplices to simplices, then the triangulations are said to be isomorphic. If $\sigma$ merely maps simplices to unions of simplices, then $\tau_{L}$ is said to be a refinement of $\tau_{K}$.

Moreover, if there exists triangulations that keep the boundary of the manifold $\mathcal{M}$ while being refinements of a previous triangulation, then this operation is known as interior ambient isotopy. Two triangulations are called called combinatorially equivalent if they admit refinements that are ambient isotopic. On the next subsection we will consider a systematic way of finding combinatorially equivalent triangulations that ensure that the topology of the manifold is preserved.

In Appendix B we formally pursue this topological equivalence for the case of Homology theory. 


\section{Appendix B}

\section{A brief account on Homology}

Given the set $S$ of vertices of a simplex, we define an orientation on the simplex by selecting some particular ordering of $S$. Vertex orderingns

\section{B.1 Homology Groups}

Formally, if $A^{n}=\left[v_{0}, v_{1}, \cdots, v_{n}\right]$ is an oriented $n$-simplex then the orientation of the $(n-1)$-face of $A^{n}$, with vertex set $\left\{v_{0}, \cdots, v_{i-1}, v_{i+1}, \cdots, v_{n}\right\}$ is given by

$$
F_{i}=(-1)^{-1}\left[v_{0}, \cdots, v_{i-1}, v_{i+1}, \cdots, v_{n}\right]
$$

Definition B.1.1. Given a set $A^{n} 1, \cdots, A^{n} k$ of arbitrarily oriented $n$-simplices of complex $\mathbf{K}$ and an abelian group $G$, we define an $n$-chain $x$ with coefficients in $G$ as a formal sum:

$$
x=g_{1} A^{n} 1+g_{2} A^{n} 2+\cdots+g_{k} A^{n} k,
$$

where $g_{i} \in G$.

Usually the group is taken to be $G=\mathbb{Z}$. It is clear that the set of $n$-chains forms an abelian group over addition, this is, for $x=\sum_{i=1}^{k} g_{i} A^{n} i, y=\sum_{i=1}^{k} h_{i} A^{n} i$, we havde $x+y=\sum_{i=1}^{k}\left(g_{i}+h_{i}\right) A^{n} i$. We will denote the group of $n$-chains by $L_{n}$.

Definition B.1.2. Let $A^{n}$ be an oriented $n$-simplex in a complex $\mathbf{K}$. The boundary of $A^{n}$ is defined as the $(n-1)$-chain of $\mathbf{K}$ over $\mathbb{Z}$ given by

$$
\partial\left(A^{n}\right)=A^{n-1} 0+A^{n-1} 1+\cdots+A^{n-1} n
$$


where $A^{n-1} i$ is an $(n-1)$ - face of $A^{n}$. If $n=0$, we define $\partial\left(\sigma^{0}\right)=0$.

Notice that, since $A^{n}$ was oriented, the $A^{n-1} i$ have associated orientations as well. We can extend the definition of boundary linearly to all of $L_{n}$ in the following way, for an $n$-chain $x=\sum_{i=1}^{k} g_{i} A^{n} i$, define

$$
\partial(x)=\sum_{i=1}^{k} g_{i} \partial\left(A_{i}^{n}\right)
$$

where $A^{n} i$ are the $n$-simplexes of $\mathbf{K}$. So we have that $\partial: L_{n} \rightarrow L_{n-1}$.

Definition B.1.3. An $n$-chain is called cycle if its boundary is zero. We denote the set of $n$-cycles of $\mathbf{K}$ over $\mathbb{Z}$ by $Z_{n}$. It is clear that $Z_{n}$ is a subgroup of $L_{n}$ and that satisfies the relation $Z_{n}=\operatorname{ker}(\partial)$.

It follows that, thus defined, the boundary of any simplex is a cycle, since $\partial \circ \partial=0$.

Definition B.1.4. An $n$-cycle $x$ of a $k$-complex $\mathbf{K}$ is homologous to zero if it is the boundary of an $(n+1)$-chain of $\mathbf{K}, n=0,1, \cdots, k-1$. A boundary is then any cycle that is homologous to zero. This relation is written $x \sim 0$, and the subgroup of $Z_{n}$ of boundaries is denoted $B_{n}$. We also write $B_{n}=\operatorname{im}(\partial)$.

The relation $x \sim 0$ gives an equivalence relation: for two chains $x, y,(x-y) \sim 0 \Rightarrow x \sim$ $y$, and we call $x$ and $y$ homologous. Since $B_{n}$ is a subgroup of $Z_{n}$ we may form the quotient group:

Definition B.1.5. The group $H_{n}$ is the $n$-dimensional homology group of the complex $\mathbf{K}$ over $\mathbb{Z}$, defined by

$$
H_{n}=\frac{Z_{n}}{B_{n}}=\frac{\operatorname{ker}(\partial)}{\operatorname{im}(\partial)} .
$$

Definition B.1.6. A subcomplex is a subset $S$ of the simplices of a complex $\mathbf{K}$ such that $S$ is also a complex.

The set of all simplices in a complex $\mathbf{K}$ with dimensions less than or equal to $n$ is called the $n$-skeleton of $\mathbf{K}$. From the previous definition it is clear that the $n$-skeleton is a subcomplex.

Definition B.1.7. A complex $\mathbf{K}$ is connected if it cannot be represented as the disjoint union of two or more non-empty subcomplexes. A geometric complex is path-connected if there exists a path made of 1-simplices from any vertex to any other. 
The following proposition is an important feature of the homological analysis

Proposition B.1.8. Path-connected $\Leftrightarrow$ Connected

Proof. To prove the necessary, suppose $\mathbf{K}$ is not connected. Then we can select two disjoint subcomplexes $\mathbf{L}$ and $\mathbf{M}$ such that $\mathbf{L} \sqcup \mathbf{M}=\mathbf{K}$. Assume a path exists between some vertex $l_{0} \in \mathbf{L}$ and $m_{0} \in \mathbf{M}$. But them, if $l_{i}$ is the last vertex in the path cannot be contained in either $\mathbf{L}$ or $\mathbf{M}$ or they would have a nonempty intersection, a contradiction.

To prove the sufficient, assume there exist points $l_{0}$ and $m_{0}$ in $\mathbf{K}$ with no path between them. Then we define $\mathbf{L}$ as the path-connected subcomplex which contains $m_{0}$. If $v_{0} \in \mathbf{L} \cap \mathbf{M} \neq \emptyset$, then there exists a path from $l_{0}$ to $v_{0}$ and a path from $v_{0}$ to $m_{0}$. Concatenating these paths gives a path from $l_{0}$ to $m_{0}$, a contradiction.

The following theorem formalizes the latter observations

Theorem B.1.9. If $K_{1}, \cdots, K_{p}$ is the set of all connected components of a complex $K$, and $H_{n}, H_{n_{i}}$ are the homology groups of $\mathbf{K}$ and $\mathbf{K}_{i}$, respectively, then $H_{n}$ is isomorphic to the direct sum $H_{n_{1}} \oplus \cdots \oplus H_{n_{p}}$.

Proof. Let $L_{n}$ be the group of $n$-chains of $\mathbf{K}$. and $\mathbf{K}_{i}$ the $i$-th component of $\mathbf{K}$. Denote by $L_{n_{i}}$ the group of $n$-chains of $\mathbf{K}_{i}$. It is clear that $L_{n_{i}}$ is a subgroup of $L_{n}$ and moreover, that

$$
L_{n}=L_{n_{1}} \oplus \cdots \oplus L_{n_{p}}
$$

We wish to show that a similar component wise decomposition holds for the groups $B_{n}$ and $Z_{n}$. If we let $B_{n_{i}}=\partial\left(L_{n+1_{i}}\right)$ be the image of $\partial$ restricted to the subgroup $L_{n_{i}}$, then we can represent the group $B_{n}$ by the direct sum of such restrictions

$$
B_{n}=B_{n_{1}} \oplus \cdots \oplus B_{n_{p}}
$$

so given an element $X \in L_{n+1}$, represented by

$$
\begin{array}{r}
x=x_{i}+\cdots+x_{p}, \\
\partial(x)=\partial\left(x_{i}\right)+\cdots+\partial\left(x_{p}\right) \in B_{n},
\end{array}
$$

where $x_{i} \in L_{n+1_{i}}$.

Now, let $Z_{n_{i}}=\operatorname{ker}(\partial) \cap L_{n_{i}}$. Then

$$
Z_{n}=Z_{n_{1}} \oplus \cdots \oplus Z_{n_{p}}
$$


To verify this, we note that in order for $x \in L_{n}$ to be in $Z_{n}$, we need $\partial(x)=0$. But $\partial(x)=\partial\left(x_{1}\right)+\cdots+\partial\left(x_{p}\right)$, so $\partial(x)=0 \Rightarrow \partial\left(x_{i}\right)=0$, that is, that $x_{i} \in Z_{n_{i}}$.

Since $Z_{n}$ and $B_{n}$ both break down component wise,

$$
Z_{n} / B_{n}=Z_{n_{1}} / B_{n_{1}} \oplus \cdots \oplus Z_{n_{p}} / B_{n_{p}}
$$

and

$$
H_{n}=H_{n_{1}} \oplus \cdots \oplus H_{n_{p}}
$$

Definition B.1.10. The index of a chain $x=\sum_{i=1}^{k} g_{i} A^{n} i$ is defined as $I(x)=\sum_{i=1}^{k} g_{i}$.

Proposition B.1.11. If $\mathbf{K}$ is a connected complex, then for $x$ a 0 -chain, $I(x)=0$ is equivalent to $x \sim 0$, and $H_{0}(K, \mathbb{Z})$ is isomorphic to $\mathbb{Z}$.

Proof. We first show that $x \sim 0 \rightarrow I(x)=0$ : Let $A^{1}=\left(a_{0}, a_{1}\right)$ be a 1 -simplex. Then

$$
x=\partial\left(g A^{1}\right)=g a_{1}-g a_{0},
$$

but $x=\partial\left(g A^{1}\right) \rightarrow x \sim 0$, and we can see that $I(x)=I\left(g A^{1}\right)=g-g=0$. Since $I(x+y)=I(x)+I(y), I$ is a homomorphism, and any $y \in L^{1}$ is of the form $\sum_{i=0}^{q} g_{i} A^{1} i$, where $A^{1} i=\left(a_{i}, a_{i+1}\right)$, we have

$$
x=\partial(y) \sim 0 \Rightarrow I(x)=I(\partial(y))=0 .
$$

The necessary condition follows, we take $v$ and $w$ to be two vertices of $\mathbf{K}$. $\mathbf{K}$ is connected, so there exists a path between them consisting of 1 -simplices $A^{1} i=\left(a_{i}, a_{i+1}\right), i=$ $0, \cdots, q-1$, where $a_{0}=v$ and $a_{q}=w$. We consider the boundary of the chain $y=$ $\sum_{i=0}^{q} g A^{1} i$, given by

$$
\partial(y)=\sum_{i=0}^{q} g \partial\left(A^{1} i\right)=\sum_{i=0}^{q} g\left[\left(a_{i+1}\right)-\left(a_{i}\right)\right]=g w-g v
$$

with $I(\partial(y))=0$. $\partial(y)$ is a boundary, so $x=\partial(y) \sim 0 \Rightarrow(g w-g v) \sim 0 \Rightarrow g w \sim g v$, and this implies that any 0 -chain $x$ of $\mathbf{K}$ is homologous to the chain $g v$. As $x \sim 0 \Rightarrow$ $I(x)=0$, we see that homologous chains have equal indexes. Thus, $I(x)=I(g v)=g$. Then, we have $x \sim g v \Rightarrow x \sim I(x) v$, but this shows that if $I(x)=0 \Leftrightarrow x \sim 0$. 
As noted, $I$ is a homomorphism of $L_{0}=Z_{0}$ into $\mathbb{Z}$. For $x$ a 0 -simplex and $g \in \mathbb{Z}$, $g x \in L_{0}$ is a cycle with $I(g x)=g$. Hence, $I\left(Z_{0}\right)=\mathbb{Z}$. As $I(x)=0 \Leftrightarrow x \sim 0$, we have $B_{0}=\operatorname{ker}(I)$, so that $H_{0}=Z_{0} / B_{0}=\mathbb{Z}$.

Theorem B.1.12. The zero-dimensional homology group of a complex $\mathbf{K}$ over $\mathbb{Z}$ is isomorphic to $\mathbb{Z}^{p}=\oplus_{p} \mathbb{Z}$ where $p$ is the number of connected components of $\mathbf{K}$.

Proof. This follows directly from Theorem B.1.9 and Proposition B.1.11.

\section{B.2 Singular Homology}

A particularly powerful tool in demonstrating that two topological spaces are the same is by the use of singular homology, which we pass on to define.

Definition B.2.1. Given a topological space $X$, a singular $n$-simplex in $X$ is a map $\sigma: \sigma^{n} \rightarrow X$, such that $\gamma$ is continuous.

Definition B.2.2. Let $C_{n}(X)$ be the free abelian group with basis the set of singular $n$-simplices of $X$. Elements of $C_{n}(X)$ are called singular singular $n$-chains. These are finite formal sums $\sum_{i} g_{i} \sigma_{i}$, where $g_{i} \in \mathbb{Z}$.

Definition B.2.3. The boundary map $\delta_{n}: C_{n}(X) \rightarrow C_{n-1}(X)$ is given by:

$$
\delta_{n}(\sigma)=\left.\sum_{i}(-1) \sigma\right|_{\left[v_{0}, \cdots, v_{i-1}, v_{i+1}, \cdots, v_{n}\right]}
$$

where $v_{i}$ are the 0 -simplices of $\sigma$, that is, the maps of the vertices of $\sigma^{n}: v_{i}: \sigma^{0} \rightarrow X$.

Notice that, in this case, the relation $\delta_{n} \circ \delta_{n+1}=0$ follows, as expected.

Definition B.2.4. The singular homology group $H_{n}(X)$ is defined to be the quotient $H_{n}(X)=\operatorname{ker}\left(\delta_{n}\right) / \operatorname{im}\left(\delta_{n+1}\right)$.

The singular homology is definitely simpler to calculate that homology itself, in the following sections we will briefly demonstrate that both notions are in fact equivalent.

Proposition B.2.5. Given a topological space $X, H_{n}(X)$ is isomorphic to the direct sum $H_{n}\left(X_{1}\right) \oplus H_{n}\left(X_{2}\right) \oplus \cdots \oplus H_{n}\left(X_{p}\right)$, where $X_{i}$ are the path-connected components of $X$.

Proof. Since the maps $\sigma$ are continuous, a singular simplex always has a path-connected image in $X$. Hence, $C_{n}(X)$ can be written as the direct sum of subgroups $C_{n}\left(X_{1}\right) \oplus \cdots \oplus$ 
$C_{n}\left(X_{p}\right)$. The boundary map $\delta$ is a homomorphism, so it preserves this decomposition. Hence, $\operatorname{ker}\left(\delta_{n}\right)$ and $\operatorname{im}\left(\delta_{n-1}\right)$ also split, and we have $H_{n}(X) \cong H_{n}\left(X_{1}\right) \oplus \cdots \oplus H_{n}\left(X_{p}\right)$.

\section{B.3 Chain complexes, exact sequences and relative homol- ogy}

Definition B.3.1 (chain complexes). A chain complex is a sequence of abelian groups $C_{n}$ connected by homomorphisms $\delta_{n}$ called boundary operators, such that the composition of any two consecutive maps is $\delta_{n} \circ \delta_{n+1}=0$, as showed

$$
\cdots C_{n+1} \stackrel{\delta_{n+1}}{\longrightarrow} C_{n} \stackrel{\delta_{n}}{\longrightarrow} C_{n-1} \rightarrow \cdots \rightarrow C_{1} \stackrel{\delta_{1}}{\longrightarrow} C_{0} \stackrel{\delta_{0}}{\longrightarrow} C_{0}
$$

Definition B.3.2 (Homology). The homology groups of a chain complex are given by $\operatorname{ker}\left(\delta_{n}\right) / \operatorname{im}\left(\delta_{n+1}\right)$.

Definition B.3.3 (Chain map). A chain map $f$ between two chain complexes $A, \delta_{A}$ and $B, \delta_{B}$ is a collection of maps $f_{n}: A_{n} \rightarrow B_{n}$ such that $f$ commutes with the operators $\delta_{A}$ and $\delta_{B}$ as in the following diagram

$$
\begin{aligned}
& \ldots \rightarrow A_{n+1} \stackrel{\delta_{n+1}^{A}}{\longrightarrow} A_{n} \stackrel{\delta_{n}^{A}}{\longrightarrow} A_{n-1} \rightarrow \ldots \\
& f_{n+1} \downarrow \\
& \cdots \rightarrow B_{n+1} \underset{\delta_{n+1}^{B}}{\longrightarrow} B_{n} \underset{\delta_{n}^{B}}{\longrightarrow} B_{n-1} \rightarrow \ldots
\end{aligned}
$$

Figure B.1: a chain map

Theorem B.3.4. A chain map between two chain complexes induces homomorphisms between homology groups

Proof. As shown in Figure B.1, we have $f_{n} \delta_{n}^{A}=\delta_{n+1}^{B} f_{n+1}$. Then $f_{n}$ maps cycles to cycles and boundaries to boundaries, so $f$ induces a homomorphism $f_{*}: H_{n}(A) \rightarrow H_{n}(B)$.

Definition B.3.5 (exact sequences). A sequence of the form:

$$
\cdots \rightarrow A_{n+1} \stackrel{\alpha_{n+1}}{\longrightarrow} A_{n} \stackrel{\alpha_{n}}{\longrightarrow} A_{n-1} \rightarrow \cdots
$$

where the $A_{i}$ are abelian groups and the $\alpha_{i}$ are homeomorphisms is called exact sequence if $\operatorname{ker}\left(\alpha_{n}\right) / \operatorname{im}\left(\alpha_{n+1}\right)$, for all $n$. 
We note two things:

(1) $\operatorname{ker}\left(\alpha_{n}\right) / \operatorname{im}\left(\alpha_{n+1}\right) \Rightarrow \operatorname{im}\left(\alpha_{n+1}\right) \subset \operatorname{ker}\left(\alpha_{n}\right) \Leftrightarrow \alpha_{n} \circ \alpha_{n+1}=0$, so an exact sequence ifs a chain complex.

(2) As ker $\left(\alpha_{n}\right) \subset \operatorname{im}\left(\alpha_{n+1}\right)$, the homology groups of an exact sequence are trivial.

From this, easily follows that:

(a) $0 \rightarrow A \stackrel{a}{\rightarrow} B$ is exact $\Leftrightarrow \operatorname{ker}(a)=0$, or $a$ is injective.

(b) $A \stackrel{a}{\rightarrow} B \rightarrow 0$ is exact $\Leftrightarrow \operatorname{im}(a)=0$, or $a$ is surjective.

(c) $0 \rightarrow A \stackrel{a}{\rightarrow} B \rightarrow 0$ is exact iff $a$ is an isomorphism.

(d) $0 \rightarrow A \stackrel{a}{\rightarrow} B \stackrel{b}{\rightarrow} C \rightarrow 0$ is exact iff $a$ is injective, $b$ is surjective and $\operatorname{ker}(b)=\operatorname{im}(a)$ in which case $b$ gives an isomorphism $C \cong B / \operatorname{im}(a)$. Furthermore, if $a: \hookrightarrow B$, an inclusion, then $C \cong B / A$. This type of exact sequence is called a short exact sequence.

Definition B.3.6 (Relative homology groups). Given a space $X$ and a subspace $A \subset X$, define $C_{n}(X, A)$ to be the quotient group $C_{n}(X) / C_{n}(A)$. This means that chains in $A$ get identified with the trivial chains in $C_{n}(X)$. Since the operator $\delta: C_{n}(X) \rightarrow$ $C_{n-1}(X)$ also takes $\delta: C_{n}(A) \rightarrow C_{n-1}(A)$, we get a natural boundary map on the quotient group $\delta: C_{n}(X, A) \rightarrow C_{n-1}(X, A)$, which give us the chain complex

$$
\cdots \rightarrow C_{n+1}(X, A) \stackrel{\delta_{n+1}}{\longrightarrow} C_{n}(X, A) \stackrel{\delta_{n}}{\longrightarrow} C_{n-1}(X, A) \rightarrow \cdots
$$

which is a chain complex because $\delta_{n+1} \circ \delta_{n}=0$. We define the relative homology groups $H_{n}(X, A)$ as the homology of this chain complex.

The following facts can then be established:

(a) The elements in $H_{n}(X, A)$ are represented by relative cycles, or $n$-chains $x$ in $C_{n}(X)$ such that $\delta_{n} x=C_{n+1}(X)$.

(b) A relative cycle $x$ is trivial iff it is a relative boundary, i.e. $x$ is the sum of a chain in $C_{n}(A)$ and the boundary of a chain in $C_{n+1}(X)$.

We will show that the relative homology groups $H_{n}(X, A)$ fit into the long exact sequence

$$
\cdots \rightarrow H_{n}(A) \rightarrow H_{n}(X) \rightarrow H_{n}(X, A) \rightarrow H_{n-1}(A) \rightarrow \cdots \rightarrow H_{0}(X, A) \rightarrow 0
$$


in an algebraically manner.

We start by considering the diagram of Figure B.2

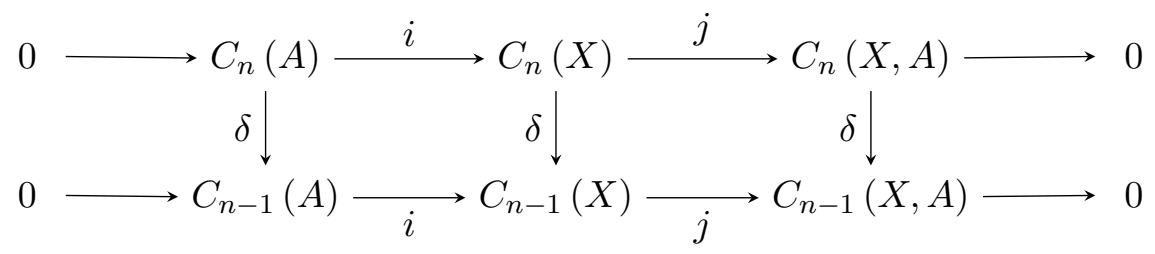

FIGURE B.2

where $i$ is the inclusion map $C_{n}(A) \hookrightarrow C_{n}(X)$, and $j$ is the quotient map $C_{n}(X) \rightarrow$ $C_{n}(X, A)$. The diagram is commutative, so we can perform the following concatenation

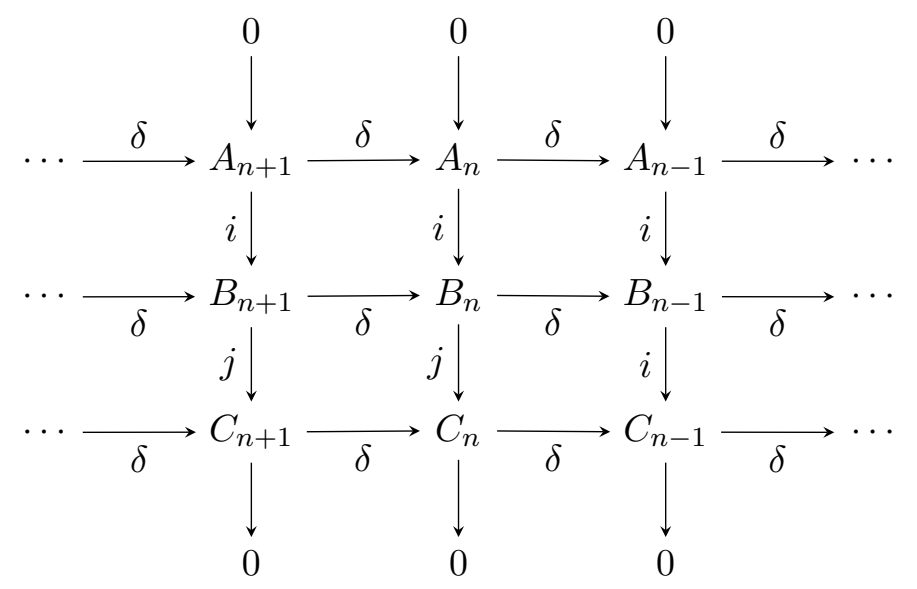

FigURE B.3

where the columns are short exact sequences and the rows are chain complexes of abelian groups $A_{i}, B_{i}$ and $C_{i}$. Notice that the diagram implies that $i$ and $j$ are chain maps, and so induce maps $i_{*}$ and $j_{*}$ on homology. We take some $c \in C_{n}$ to be cycle. Since $j$ is surjective, we have $c=j(b)$ for some $b \in B_{n}$. For $\delta b \in B_{n-1}, j(\delta b)=\delta j(b)$ by commutativity. Hence, $\delta j(b)=\delta c=0$, since $c$ is a cycle. Thus $\delta b \in \operatorname{ker}(j)$. Since the columns are exact, we have $\operatorname{ker}(j)=\operatorname{im}(i)$, therefore $\delta(b)=i(a)$ for some $a \in A_{n-1}$.nCommutativity gives $i(\delta(a))=\delta i(a)=\delta \delta b=0$, and so $i$ is injective $\Rightarrow \delta(a)=0$. Therefore $a$ is a cycle and represents an element $[a] \in H_{n-1}(A)$ of homology. We can now define $\delta: H_{n}(C) \rightarrow H_{n-1}(A)$ by sending the homology class of $[c]$ to the homology class of $[a], \delta[c]=[a]$. The latter construction is well-defined for the following reasons:

(1) $i$ is injective. Then $a$ is uniquely determined by $\delta b$. 
(2) Choosing $b^{\prime}$ instead of $b$ gives $j\left(b^{\prime}\right)=j(b) \Rightarrow j\left(b^{\prime}\right)-j(b)=0 \Rightarrow j\left(b^{\prime}-b\right)=$ $0 \Rightarrow b-b^{\prime} \in \operatorname{ker}(j)=\operatorname{im}(i)$. Hence, $b-b^{\prime}=i\left(a^{\prime}\right)$, or $b^{\prime}=b+i(a)$, and $\delta(b+i(a))=\delta(b)+\delta i\left(a^{\prime}\right)=i(a)+i \delta\left(a^{\prime}\right)=i\left(a+\delta a^{\prime}\right)$. But $\delta a^{\prime} \sim 0$, so $a+\delta a^{\prime} \sim a$.

(3) Choosing $c_{*}$ from the coset from the coset of $c$ implies $c_{*}=c+\delta c^{\prime} . c^{\prime}=j\left(b^{\prime}\right)$ for some $b^{\prime}$, then $c+\delta c^{\prime}=c+\delta j\left(b^{\prime}\right)=j(b)+j \delta\left(b^{\prime}\right)=j\left(b+\delta b^{\prime}\right)$. Thus changing $c$ has the effect of changing $b$ to a homologous element, which does not affect $a$ at all.

Proposition B.3.7. The map $\delta: H_{n}(C) \rightarrow H_{n-1}(A)$ defined above is a homomorphism.

Proof. If $\delta\left[c_{1}\right]=\left[a_{1}\right]$ and $\delta\left[c_{2}\right]=\left[a_{2}\right]$ via $b_{1}$ and $b_{2}$, as above, then we have $j\left(b_{1}+b_{2}\right)=$ $j\left(b_{1}\right)+j\left(b_{2}\right)=c_{1}+c_{2}$, and $i\left(a_{1}+a_{2}\right)=i\left(a_{1}\right)+i\left(a_{2}\right)=\delta\left(b_{1}\right)+\delta\left(b_{2}\right)=\delta\left(b_{1}+b_{2}\right)$. Hence, $\delta\left(\left[c_{1}\right]+\left[c_{2}\right]\right)=\left[a_{1}\right]+\left[a_{2}\right]$.

Proposition B.3.8. The sequence

$$
\cdots \rightarrow H_{n}(A) \stackrel{i_{*}}{\rightarrow} H_{n}(B) \stackrel{j_{*}}{\rightarrow} H_{n}(C) \stackrel{\delta}{\rightarrow} H_{n-1}(A) \stackrel{i_{*}}{\rightarrow} H_{n-1}(B) \rightarrow \cdots
$$

is exact.

Proof. There are six inclusions to be verified:

(1) $\operatorname{im}\left(i_{*}\right) \subset \operatorname{ker}\left(j_{*}\right): j i=0 \Rightarrow j_{*} i_{*}=0$.

(2) $\operatorname{im}\left(j_{*}\right) \subset \operatorname{ker}(\delta): \delta b=0$ by definition, so $\delta j_{*}=0$.

(3) $\operatorname{im}(\delta) \subset \operatorname{ker}\left(i_{*}\right): i_{*} \delta=0$ since $i_{*} \delta[c]=[\delta b]=0$

(4) $\operatorname{ker}\left(j_{*}\right) \subset \operatorname{im}\left(i_{*}\right):$ A homology class in $\operatorname{ker}\left(j_{*}\right)$ can be represented by a cycle $b \in B_{n}$ such that $j(b)=\delta c^{\prime}$ is a boundary for some $c^{\prime} \in C_{n+1}$. Surjectivity of $j$ gives $c^{\prime}=j\left(b^{\prime}\right)$ for some $b^{\prime} \in B_{n+1}$. But then $j(b)=\delta c^{\prime}=\delta j b^{\prime}=0$, and $b-\delta b^{\prime}=i(a)$ for some $a \in A_{n} . a$ is cycle, since $i \delta a=\delta i a=\delta\left(b-\delta b^{\prime}\right)=\delta b=0$ because $b$ is a cycle and $i$ injective. Therefore, $i_{*}[a]=[b]$, and the two inclusions give us $\operatorname{im}\left(i_{*}\right)=\operatorname{ker}\left(j_{*}\right)$.

(5) $\operatorname{ker}(\delta) \subset \operatorname{im}\left(j_{*}\right)$ : We take $c$ a representative of a homology class in $\operatorname{ker}(\delta)$. Then we have $a=\delta a^{\prime}$ for some $a^{\prime} \in A_{n} . \quad b-i\left(a^{\prime}\right)$ is a cycle because $\delta\left(b-i\left(a^{\prime}\right)\right)=$ $\delta(b)-\delta i\left(a^{\prime}\right)=\delta b-i\left(\delta^{\prime} a\right)=\delta b-i(a)=0$. We also have $j\left(b-i\left(a^{\prime}\right)\right)=j(b)-$ $j i\left(a^{\prime}\right)=j(b)=c$, so $\operatorname{ker}(\delta) \subset \operatorname{im}\left(j_{*}\right)$. 
(6) $\operatorname{ker}\left(i_{*}\right) \subset \operatorname{im}(\delta)$ : We take a cycle $a \in A_{n-1}$ such $i(a)=\delta b$ for some $b \in B_{n}$. $j(b)$ is a cycle, because $\delta(j(b))=j(\delta b)=j i(a)=0$. Thus $\delta[j(b)]=[a]$. Then $\operatorname{ker}\left(i_{*}\right) \subset \operatorname{im}(\delta)$.

Hence, $\operatorname{im}\left(i_{*}\right)=\operatorname{ker}\left(j_{*}\right), \operatorname{im}\left(j_{*}\right)=\operatorname{im}(\delta)$ and $\operatorname{im}(\delta)=\operatorname{ker}\left(i_{*}\right)$, that holds for a exact sequence.

Proposition B.3.9. The sequence:

$$
\cdots \rightarrow H_{n}(A) \stackrel{i_{*}}{\rightarrow} H_{n}(X) \stackrel{j_{*}}{\rightarrow} H_{n}(X, A) \stackrel{\delta}{\rightarrow} H_{n-1}(A) \rightarrow \cdots \rightarrow H_{0}(X, A) \rightarrow 0
$$

is exact.

Proof. The proof is analogous to the one given in the previous proposition with the remark that for a relative cycle $x$ in $H_{n}(X, A), \delta[x]$ is the class of the cycle $[\delta x] \in$ $H_{n-1}(A)$.

The following is a standard theorem that will be stated but not proved since its proof is out of the scope of this section.

Theorem B.3.10 (Excision Theorem). Given $Y \subset A \subset X$, with the closure of $Y$ contained in the interior of $A$, then $(X-Y, A-Y) \hookrightarrow(X, A)$ induces isomorphisms $H_{n}(X-A, A-Y) \rightarrow H_{n}(X, A)$ for all $n$.

Although not all topological spaces are homeomorphic to a simplicial complex, for the purpose of this work, every space we consider can be analyzed as a simplicial complex. In order to show the equivalence between singular and simplicial homology, we need to show the existence of an isomorphism between the two groups for all $n$. It is easy enough to see the existence of a homomorphism, recall the map $L_{n}(X) \rightarrow C_{n}(X)$ from the simplicial chain group to the singular chain group which sends each simplex of $X$ to $\sigma: \sigma^{n} \rightarrow X$. This induces a map between the singular and simplicial homology. We will use the following lemma:

Lemma B.3.11. In a commutative diagram of the form shown in Figure B.4 if $\alpha, \beta, \delta$,

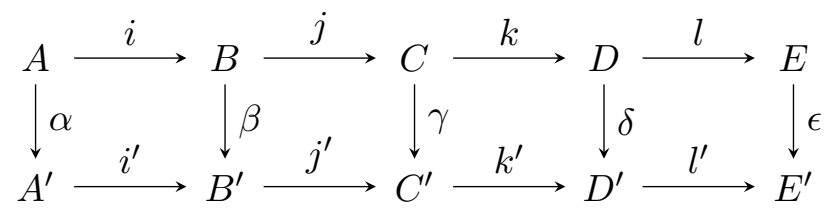

FiguRe B.4

and $\epsilon$ are all isomorphisms, and the two rows are exact, then $\gamma$ is also an isomorphism. 
Proof. Commutativity of the diagram gives us that $\gamma$ must be a homomorphism. Therefore, it suffices to show that $\gamma$ is a bijection. Take $c^{\prime} \in C^{\prime}$. Since $\delta$ is surjective, $k^{\prime}\left(c^{\prime}\right)=$ $\delta(d)$ for some $d \in D$. Injectivity of $\epsilon$ gives us that $\epsilon l(d)=l^{\prime} k^{\prime}\left(c^{\prime}\right)=0 \Rightarrow l(d)=0$. But the rows are exact, so we have $d=k(c)$ for some $c \in C$.

$k^{\prime}\left(c^{\prime}\right)-k^{\prime}(\gamma(c))=k^{\prime}\left(c^{\prime}\right)-\delta k(c)=k^{\prime}\left(c^{\prime}\right)-\delta(d)=0$, so $k^{\prime}\left(c^{\prime}-\gamma(c)\right)=0$, and, by exactness, $c^{\prime}-\gamma(c)=j^{\prime}\left(b^{\prime}\right)$ for $b^{\prime} \in B^{\prime}$. Surjectivity of $\beta$ gives $b^{\prime}=\beta(b)$ for some $b \in B$, so $\gamma(c+j(b))=\gamma(c)+\gamma(j(b))=\gamma(c)-j^{\prime}\left(b^{\prime}\right)=c^{\prime}$, so $\gamma$ is surjective.

For injectivity, suppose $\gamma(c)=0 . \delta$ is injectivity, so $\delta(k(c))=k^{\prime}(\gamma(c))=0 \rightarrow k(c)=0$. Then $c=j(b)$ for some $b \in B . \gamma(c)=\gamma(j(b))=j^{\prime}(\beta(b))$, so we have $\beta(b)=i^{\prime}\left(a^{\prime}\right)$ for some $a^{\prime} \in A^{\prime}$. Surjectivity of $\alpha$ gives $a^{\prime}=\alpha(a)$ for some $a \in A$. $\beta$ is injective, so $\beta(i(a)-b)=\beta(i(a))-\beta(b)=i^{\prime}(\alpha(a))-\beta(b)=i^{\prime}\left(a^{\prime}\right)-\beta(b)=0 \Rightarrow i(a)-b=0$. That is, $b=i(a)$, so $c=j(b)=j(i(a))=0$ by exactness of rows. Hence, $\gamma$ has trivial kernel and is therefore injective.

Theorem B.3.12 (Equivalence between singular and simplicial homology). For all $n$, the homomorphisms $H_{n}(X) \rightarrow \operatorname{ker}\left(\delta_{n}\right) / \mathrm{im}\left(\delta_{n-1}\right)$ are isomorphism. Thus the singular and simplicial homology groups are equivalent.

Proof. We take $X$ to be a simplicial complex. For $X^{k}$ the $k$-skeleton of $X$, we get the following commutative diagram of exact sequences, since $X^{k-1} \subset X^{k}$. For the sake of clarity, let us denote the simplicial homology by $H_{n}^{\Delta}$ to differentiate it from the singular homology $H_{n}$, then we have Figure B.5:

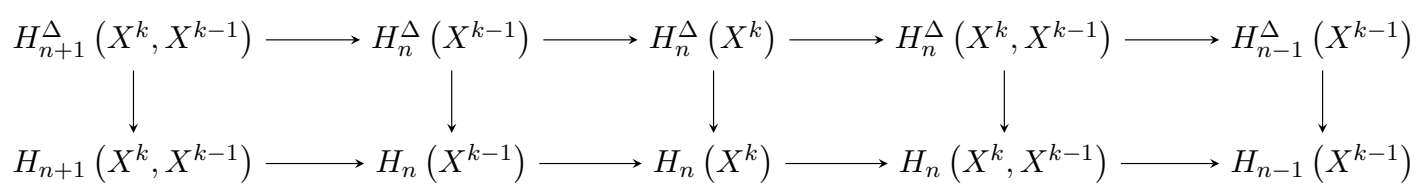

FiguRe B.5

The space $X^{k} / X^{k-1}$ contains only simplices of dimension $k$. Hence, for $n \neq k$, the group $L_{n}\left(X^{k}, X^{k-1}\right)$ is equal to zero. When $n=k, L_{n}\left(X^{k}, X^{k-1}\right)$ is a free abelian group with basis consisting of the $k$-simplices of $X$. Since the cycles $Z_{n}$ form a subgroup in $L_{n}$, and the boundary group $B_{n}$ is empty, $H_{n}^{\Delta}\left(X^{k}, X^{k-1}\right)$ has the same description as $L_{n}$, with the caveat that when $n=k$, the basis of $Z_{n}$ consists of $k$-cycles.

We observe that the characteristic maps $\sigma^{n} \rightarrow X$ for all the $k$-simplices of $X$ give us a map $\Phi: \sqcup_{i}\left(\sigma_{i}^{k}, \sigma_{i}^{k-1}\right) \rightarrow\left(X^{k}, X^{k-1}\right)$. It is then fairly clear that this map induces a homeomorphism $\Phi_{*}: \sqcup_{i} \sigma_{i}^{k} / \sqcup \sigma_{i}^{k-1} \rightarrow X^{k} / X^{k-1}$. But then, $H_{n}\left(\sqcup_{i} \sigma_{i}^{k} / \sqcup \sigma_{i}^{k-1}\right) \simeq$ $H_{n}\left(X^{k} / X^{k-1}\right)$. 
It is a consequence of the Theorem B.3.10 that there exits an isomorphism $H_{n}(X, A) \rightarrow$ $H_{n}(X / A)$ for all good pairs $(X, A)$. Then, we have

$$
H_{n} \sqcup_{i}\left(\sigma_{i}^{k}, \sigma_{i}^{k-1}\right) \simeq H_{n}\left(\sqcup_{i} \sigma_{i}^{k} / \sqcup \sigma_{i}^{k-1}\right) \quad \text { and } \quad H_{n}\left(X^{k}, X^{k-1}\right) \simeq H_{n}\left(X^{k} / X^{k-1}\right) .
$$

By transitivity, this gives us $H_{n} \sqcup_{i}\left(\sigma_{i}^{k}, \sigma_{i}^{k-1}\right) \simeq H_{n}\left(X^{k}, X^{k-1}\right)$.

This gives us that $H_{n}\left(X^{k}, X^{k-1}\right)$ is zero $n \neq k$ and a free abelian group with basis the relative cycles given by the maps $\sigma^{n} \rightarrow X$. Therefore the map $H_{n}^{\Delta}\left(X^{k}, X^{k-1}\right) \rightarrow$ $H_{n}\left(X^{k}, X^{k-1}\right)$ is an isomorphim. That is, the first and and fourth vertical maps in the diagram are isomorphisms. Induction and Lemma B.3.11 can be used to complete the argument, giving $H_{n}^{\Delta}\left(X^{k}\right) \rightarrow H_{n}\left(X^{k}\right)$ is also an isomorphism. As was to be shown. 


\section{Appendix C}

\section{Topics in Algebraic Topology}

This Appendix exposes the main mathematical structures used to define the topological model studied.

\section{C.1 The universal coefficient Theorem}

We start by considering the split exact sequence

$$
0 \rightarrow A \rightarrow B \rightarrow C \rightarrow 0
$$

this is, there is an isomorphism $B \simeq A \oplus C$ such that the following diagram commutes:

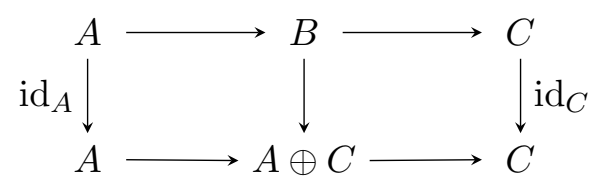

Then, for any group $G$, the sequence

$$
0 \rightarrow A \otimes G \rightarrow B \otimes G \rightarrow C \otimes G \rightarrow 0
$$

is also exact. This suggest that we can define the nnew chain complex

$$
C_{*}(X, G):=C_{*}(X) \otimes G,
$$

and

$$
H_{p}(X, G):=H_{p}\left(C_{*}(X) \otimes G\right)
$$


We will make sense of this definitions along the section.

Let $(X, A)$ be a pair of spaces, then

Lemma C.1.1. The long exact sequence of chain complexes

$$
o \rightarrow C_{*} A \rightarrow C_{*} X \rightarrow C_{*}(X, A) \rightarrow 0
$$

is split.

Proof. We have $\sigma^{p} X=\sigma^{p} A \cup R$, where $R=\left\{f: \sigma^{p} \rightarrow X \mid f\left(\sigma^{p}\right) \nsubseteq A\right\}$. Thus $C_{p} X=$ $\mathbb{Z} \sigma^{p} X=\left(\mathbb{Z} \sigma^{p} A\right) \oplus \mathbb{Z} R$. Sending all of $R$ to zero and elements of $\sigma^{p} A$ to themselves defines a splitting $C_{p} X \rightarrow C_{p} A$.

Using the previous Lemma, we find that

$$
0 \rightarrow C_{*}(A, G) \rightarrow C_{*}(X, G) \rightarrow C_{*}(X, A ; G) \rightarrow 0
$$

is still exact. Consequently, we have

Corollary C.1.2. A pair $(X, A)$ gives rise to a long exact sequence

$$
\cdots \rightarrow H_{p}(A, G) \rightarrow H_{p}(X, G) \rightarrow H_{p}(X, A ; G) \rightarrow H_{p-1}(A, G) \rightarrow \cdots
$$

In general, the structure of homology will not be affected by the tensor product with $G$, this is, we also have homotopy invariance, excision, and good behavior with respect to coproducts (i.e. Mayer -Vietoris)

On the other hand, if

$$
0 \rightarrow A \rightarrow B \rightarrow C \rightarrow 0
$$

is a short exact sequence, then

$$
0 \rightarrow \operatorname{hom}(C, G) \rightarrow \operatorname{hom}(B, G) \rightarrow \operatorname{hom}(A, G)
$$

is exact, but the last arrow is not necessarily surjective. Furthermore, if the sequence C.1 is also split, then

$$
\operatorname{hom}(B, G) \simeq \operatorname{hom}(A, G) \times \operatorname{hom}(C, G),
$$


and so $\operatorname{hom}(B, G) \rightarrow \operatorname{hom}(A, G)$ is surjective. Thus, if we define $C^{p}(X, G):=\operatorname{hom}\left(C_{p}(X), G\right)$, then Lemma C.1.1 implies that we have a short exact sequence of cochains complexes

$$
0 \rightarrow C^{*}(X, A ; G) \rightarrow C^{*}(X, G) \rightarrow C^{*}(A, G) \rightarrow 0
$$

and so a long exact sequence in cohomology

$$
\cdots \rightarrow H^{p}(X, A ; G) \rightarrow H^{p}(X, G) \rightarrow H^{p}(A, G) \rightarrow H^{p+1}(X, A ; G) \rightarrow \cdots
$$

And as before, the structure of cohomology pass through hom $(-, G)$ to give homotopy invariance, excision (i.e. Mayer- Vietoris).

We outline now the strategy to arrive at the universal coefficient theorem for chomology.

(1) We identify a class of groups, in this case the injectives, with the property that, if $I$ is injective, then

$$
H^{p}(X, I) \simeq \operatorname{hom}\left(H_{p} X, I\right) .
$$

(2) We show that if $G$ is any abelian group, then it admits an injective resolution, this is, a short exact sequence:

$$
0 \rightarrow G \rightarrow I_{0} \rightarrow I_{1} \rightarrow 0
$$

with $I_{0}$ and $I_{1}$ injective.

(3) We show that if $A$ is an abelian group and we let $\operatorname{Ext}(A, G)$ be the cokernel in the sequence

$$
0 \rightarrow \operatorname{hom}(A, G) \rightarrow \operatorname{hom}\left(A, I_{0}\right) \rightarrow \operatorname{hom}\left(A, I_{1}\right) \rightarrow \operatorname{Ext}(A, G) \rightarrow 0,
$$

then the $\operatorname{Ext}(A, G)$ depends on $A$ and $G$, and not on the resolution.

The following Lemma will be used to prove the main Theorem:

Lemma C.1.3. If $0 \rightarrow A \rightarrow B \rightarrow C \rightarrow 0$ is ashort exact sequence of abelian groups, then we have a long exact sequence in cohomology

$$
H^{p}(X, A) \rightarrow H^{p}(X, B) \rightarrow H^{p}(X, C) \rightarrow H^{p+1}(X, A) \rightarrow H^{p+1}(X, B) \rightarrow H^{p+1}(X, C)
$$


Proof. Since $C_{p} X$ is a free abelian group, the sequence

$$
0 \rightarrow C^{p}(X, A) \rightarrow C^{p}(X, B) \rightarrow C^{p}(X, C) \rightarrow 0
$$

is exact. The short exact sequence of cochain complexes gives a long exact sequence in cohomology.

Applying this into an injective resolution, we have

$$
H^{p-1}\left(X, I_{0}\right) \rightarrow H^{p-1}\left(X, I_{1}\right) \rightarrow H^{p}(X, G) \rightarrow H^{p}\left(X, I_{0}\right) \rightarrow H^{p}\left(X, I_{1}\right)
$$

Definition C.1.4 (Injectives). We say that $I$ is injective if, for every injection $A \rightarrow B$, the induced map hom $(B, I) \rightarrow \operatorname{hom}(A, I)$, is sujective.

In fact, the last definition is equivalent to say that, for every exact sequence

$$
0 \rightarrow A \rightarrow B \rightarrow C \rightarrow 0
$$

the sequence

$$
0 \rightarrow \operatorname{hom}(C, I) \rightarrow \operatorname{hom}(B, I) \rightarrow \operatorname{hom}(A, I) \rightarrow 0
$$

is also exact.

Our remaining task is to show that we can construct injective resolutions, and that $\operatorname{Ext}(A, G)$ depends on $A$ and $G$, and not the injective resolution. An observation is on demand, if $I$ is an injective abelian group then it is divisible: for all $x \in I$ and all $n \neq 0$, there exist a $y \in I$ such that $n y=x$. This allows to prove the standard result, that we only state here:

Proposition C.1.5. An abelian group is injective if and only if it is divisible, and every abelian group admits an injective resolution

$$
0 \rightarrow G \rightarrow I_{0} \rightarrow I_{1} \rightarrow 0
$$

This property illustrates immediately how to resolve finitely generated abelian groups, which is our case. So, let $R$ be a commutative ring, and let $G^{\prime}$ be an $R$-module. An injective resolution of $G$ is a long exact sequence

$$
0 \rightarrow G \rightarrow I_{0} \rightarrow I_{1} \rightarrow I_{2} \rightarrow \cdots
$$


with each $I_{k}$ injective. For a general $G$ and $R$, it may be be necessary for $I_{*}$ to go on indefinitely. The following Proposition is also stated without a proof:

Proposition C.1.6. Let $0 \rightarrow C \rightarrow J_{*}$ be an injection resolution, and let $0 \rightarrow G \rightarrow$ $R_{0} \rightarrow R_{1} \rightarrow \cdots$ be any long exact sequence. Then there is a map of complexes $R_{*} \rightarrow J_{*}$, making the diagram commute. Moreover, any two such maps are chain homotopic as

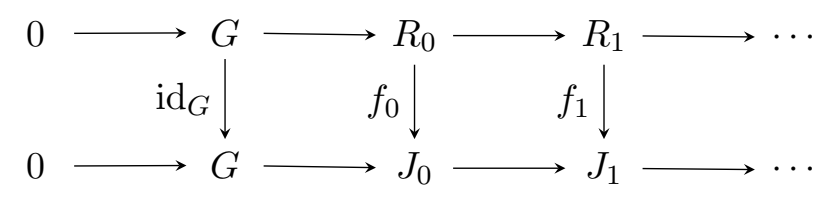

maps $R_{*} \rightarrow J_{*}$. If $0 \rightarrow G \rightarrow R_{*}$ is an injective resolution, then any map $f: R_{*} \rightarrow J_{*}$ as above participates in a chain homotopy equivalence.

It can be checked that follows from this lat Proposition that, if $G \rightarrow I_{*}$ and $G \rightarrow J_{*}$ are two injective resolutions, then $\operatorname{Ext}(A, G)$ defined using $I_{*}$ is canonically isomorphic to $\operatorname{Ext}(A, G)$ defined using $J_{*}$. The whole construction up to now leads to the universal coefficient theorem, which can then be stated as follows:

Theorem C.1.7 (Universal coefficient theorem). Consider the tensor product of modules $H_{p}(X, G) \otimes A$. The theorem states there is a short exact sequence

$$
0 \rightarrow H_{p}(X, G) \otimes A \stackrel{\mu}{\rightarrow} H_{p}(X, A) \rightarrow \operatorname{Ext}^{1}\left(H_{p-1}(X, G), A\right) \rightarrow 0 .
$$

Furthermore, this sequence splits, though not naturally. Here $\mu$ is a map induced by the bilinear map $H_{p}(X, G) \times A \rightarrow H_{p}(X, A)$.

Due to the splitting we can write the $p$-th cohomology group with coefficients in an abelian group $G$ in terms of the $p$-th and $(p-1)$-th homology groups and the group $G$ :

$$
H^{p}(X, G)=\operatorname{Hom}\left(H_{p}(X), G\right) \oplus \operatorname{Ext}^{1}\left(H_{k-1}(X), G\right) .
$$

\section{C.2 Cohomology with coefficients in a chain complex}

This section exposes the main mathematical structures used to define the topological model studied in a succinct manner. It is focused on the cohomology with coefficients in a chain complex, which will be used to find a closed expression for the ground state degeneracy over the next sections. It closely follows the treatment given by [96]. Some other technicalities are left as Appendices at the end of this paper. 
As a starting point, consider a finite abstract simplicial complex $K$ and denote by $K_{n} \subset K$ the set of n-simplexes. Let $C_{n}$ be the free abelian group with basis $K_{n}$ and $C=\left(C_{n}, \partial^{C}\right)$ the chain complex formed by these groups. Additionally, fix a chain complex of finite abelian groups $G=\left(G_{n}, \partial^{G}\right)$ so that

$$
\cdots \rightarrow C_{n} \stackrel{\partial_{n}^{C}}{\longrightarrow} C_{n-1} \rightarrow \cdots \quad \text { and } \quad \cdots \rightarrow G_{n} \stackrel{\partial_{n}^{G}}{\longrightarrow} G_{n-1} \rightarrow \cdots
$$

It is important to emphasize that the choice of a simplicial complex was done just for convenience as one could use any finite freely generated chain complex instead. Examples of alternatives would be to use a cubulation or a CW-complex as the source of chain complexes with a geometrical meaning. One might also consider chain complexes without an underlying geometry although such scenarios are yet to be explored. To summarize, the $C$ chain complex stands for the "geometrical" or "physical" content of the model whereas the $G$ chain complex of abelian groups is the mathematical structure that generalizes the notion of the gauge group. Naturally we need a generalized notion of gauge configuration, recall that in the case of discrete 1-gauge theories this corresponds to a map from the 1-simplices to the gauge group. Consequently, the equivalent of a gauge configuration in our setup consists of maps between the chain complexes $C$ and $G$. To make this more precise, consider the following technical definitions.

Definition C.2.1 $\left(\operatorname{hom}(C, G)_{p}\right)$. For all $p \in \mathbb{Z}$, let $\operatorname{hom}(C, G)_{p}$ be the following abelian group

$$
\operatorname{hom}(C, G)_{p}:=\prod_{n} \operatorname{Hom}\left(C_{n}, G_{n-p}\right)
$$

where $\operatorname{Hom}\left(C_{n}, G_{n-p}\right)$ denotes the abelian group formed by morphisms between $C_{n}$ and $G_{n-p}$. For convenience, the components of $f \in \operatorname{hom}(C, G)_{p}$ are denoted $f_{n}: C_{n} \rightarrow G_{n-p}$.

We show this construction for $f \in \operatorname{hom}(C, G)_{0}$ in FIG. C.1. Notice that in this case the maps connect each $C_{n}$ of the upper chain with its corresponding $G_{n}$ of the lower chain. In fact, the elements of $\operatorname{hom}(C, G)_{0}$ correspond to the generalized notion of gauge configuration. For $p \neq 0$, we can picture the situation as being a displacement of the images of the $f_{n}$ maps, such that they will connect each $C_{n}$ with a corresponding $G_{n-p}$ in the the lower chain. The cases when $p=-1,1$ are specially important since will be used to construct the gauge transformations and holonomy measurement operators in Chapters 4 and 5 .

We finish this section defining the main mathematical structure that will be used throughout this work. This includes a cochain complex constructed from the maps 


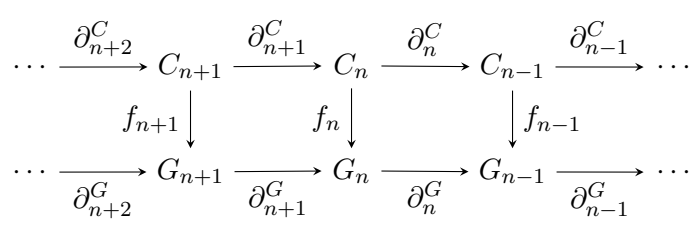

Figure C.1: Diagrammatic description for $f \in \operatorname{hom}(C, G)_{0}$. Notice that the diagrams are not necessarily commutative.

of Definition C.2.1 and its cohomology groups. We also include, at the end, a theorem by Brown [52] that will be important in Chapter 5:

Definition C.2.2 $\left(\operatorname{hom}(C, G)\right.$ cochain). Define $\operatorname{hom}(C, G)=\left(\operatorname{hom}(C, G)_{p}, \delta\right)$ as

$$
\ldots \rightarrow \operatorname{hom}(C, G)_{p} \stackrel{\delta_{p}}{\rightarrow} \operatorname{hom}(C, G)_{p+1} \rightarrow \ldots
$$

where $\delta_{p}$ is given by $\left(\delta_{p} f\right)_{n}:=f_{n-1} \partial_{n}^{C}-(-1)^{p} \partial_{n-p}^{G} f_{n}$.

One can also consider the dual groups and morphism to obtain

$$
\widehat{\operatorname{hom}}(C, G):=\left(\widehat{\operatorname{hom}}(C, G)_{p}, \widehat{\delta}\right),
$$

giving:

Definition C.2.3 $\widehat{(\widehat{\operatorname{hom}}}(C, G)$ chain). Define $\widehat{\operatorname{hom}}(C, G)=\left(\widehat{\operatorname{hom}}(C, G)_{p}, \hat{\delta}\right)$ as

$$
\cdots \leftarrow \widehat{\operatorname{hom}}(C, G)_{p} \stackrel{\hat{\delta}_{p+1}}{\longleftarrow} \widehat{\operatorname{hom}}(C, G)_{p+1} \leftarrow \ldots
$$

where $\hat{\delta}_{p+1}$ is the dual of the map $\delta_{p}$ from Definition C.2.2.

Lemma C.2.4. hom $(C, G)$ is a cochain complex and $\widehat{h o m}(C, G)$ is a chain complex.

Proof. It is straight forward from Definition C.2.2 to show that $\delta_{p+1} \circ \delta_{p} f=0$ for all $f \in \operatorname{hom}(C, G)_{p}$ so $\operatorname{hom}(C, G)$ is a cochain complex. It follows directly from Definition C.2.3 and the properties of the dualization $\hat{\delta}_{p} \circ \hat{\delta}_{p+1} t=0$ for all $t \in \widehat{\operatorname{hom}}(C, G$ ) (See Chapter 5) that $\widehat{\operatorname{hom}}(C, G)$ is a chain complex.

The latter lemma allows us to define the following cohomology groups:

Definition C.2.5 $\left(H^{p}(C, G)\right)$. The $p$-th cohomology of $C$ with coefficients in $G$ is defined as the $p$-th cohomology of $\operatorname{hom}(C, G)$, that is

$$
H^{p}(C, G)=\operatorname{ker}\left(\delta_{p}\right) / \operatorname{im}\left(\delta_{p-1}\right)
$$

where $\operatorname{ker}\left(\delta_{p}\right)$ is the kernel of the map $\delta_{p}$ and $\operatorname{im}\left(\delta_{p-1}\right)$ is the image of the map $\delta_{p-1}$. 


$$
\begin{aligned}
& \left(\delta_{p+1} \delta_{p} f\right)_{n}= \\
& \left(\delta_{p} f\right)_{n-1} \partial_{n}^{C}-(1)^{p+1} \partial_{n-p-1}^{G}\left(\delta_{p} f\right)_{n}= \\
& \left\{f_{n-2} \partial_{n-1}^{C}-(-1)^{p} \partial_{n-1-p}^{G} f_{n-1}\right\} \partial_{n}^{C}+ \\
& \quad-(1)^{p+1} \partial_{n-p-1}^{G}\left\{f_{n-1} \partial_{n}^{C}-(-1)^{p} \partial_{n-p}^{G} f_{n}\right\}= \\
& f_{n-2} \partial_{n-1}^{C} \partial_{n}^{C}-\partial_{n-p-1}^{G} \partial_{n-p}^{G} f_{n}+ \\
& \quad-(-1)^{p}\left\{\partial_{n-1-p}^{G} f_{n-1} \partial_{n}^{C}-\partial_{n-p-1}^{G} f_{n-1} \partial_{n}^{C}\right\}=0
\end{aligned}
$$

As we will see, the cohomology $H^{p}(C, G)$ is of special interest. This due to following theorem by Brown [52]:

Theorem C.2.6. For all chain complexes $C, G$ the groups

$$
H^{p}(C, G) \quad \text { and } \quad \prod_{n} H^{n}\left(C, H_{n-p}(G)\right)
$$

are isomorphic. The isomorphism is natural in $C$.

We will merely mention the theorem here, since its proof is out of the scope of this work. For a somewhat complementary reading, as well as a discussion about the associated classifying space for this cohomolgy, we refer to Møgelberg [96]. 


\section{Bibliography}

[1] F. Strocchi, "Symmetries, Symmetry Breaking, Gauge Symmetries," ArXiv eprints, Feb. 2015.

[2] Z. Nussinov, C. D. Batista, and E. Fradkin, "Intermediate Symmetries in Electronic Systems:. Dimensional Reduction, Order Out of Disorder, Dualities, and Fractionalization," International Journal of Modern Physics B, vol. 20, pp. 5239-5249, 2006.

[3] H. Georgii, Gibbs Measures and Phase Transitions. No. v. 9 in De Gruyter studies in mathematics, W. de Gruyter, 1988.

[4] "Complete list of $1 \mathrm{~d}$ landau's works," Physics-Uspekhi, vol. 41, no. 6, p. 621, 1998.

[5] R. B. Laughlin, "Anomalous quantum hall effect: An incompressible quantum fluid with fractionally charged excitations," Phys. Rev. Lett., vol. 50, pp. 1395-1398, May 1983.

[6] Z. Nussinov and G. Ortiz, "Sufficient symmetry conditions for Topological Quantum Order," Proceedings of the National Academy of Science, vol. 106, pp. 16944-16949, Sept. 2009.

[7] H. B. Thacker and G. Wong, "Bosonization and the Berry connection in twodimensional QED,” prd, vol. 91, p. 065009, Mar. 2015.

[8] W. Zhu, S. S. Gong, F. D. M. Haldane, and D. N. Sheng, "Topological Characterization of Non-Abelian Moore-Read State using Density-Matrix Renormailzation Group," ArXiv e-prints, Dec. 2014.

[9] E. Witten, "Topological quantum field theory," Communications in Mathematical Physics, vol. 117, no. 3, pp. 353-386, 1988.

[10] M. Atiyah, "Topological quantum field theories," Publications Mathématiques de l'Institut des Hautes Études Scientifiques, vol. 68, no. 1, pp. 175-186, 1988.

[11] R. Dijkgraaf and E. Witten, "Topological gauge theories and group cohomology," Communications in Mathematical Physics, vol. 129, no. 2, pp. 393-429, 1990. 
[12] Z.-C. Gu and X.-G. Wen, "Tensor-entanglement-filtering renormalization approach and symmetry-protected topological order," Phys. Rev. B, vol. 80, p. 155131, Oct 2009.

[13] X. Chen, Z.-C. Gu, Z.-X. Liu, and X.-G. Wen, "Symmetry-protected topological orders in interacting bosonic systems," Science, vol. 338, no. 6114, pp. 1604-1606, 2012.

[14] F. Pollmann, A. M. Turner, E. Berg, and M. Oshikawa, "Entanglement spectrum of a topological phase in one dimension," Phys. Rev. B, vol. 81, p. 064439, Feb 2010.

[15] F. Pollmann, E. Berg, A. M. Turner, and M. Oshikawa, "Symmetry protection of topological phases in one-dimensional quantum spin systems," Phys. Rev. B, vol. 85, p. 075125 , Feb 2012.

[16] L. Fidkowski and A. Kitaev, "Topological phases of fermions in one dimension," Phys. Rev. B, vol. 83, p. 075103, Feb 2011.

[17] M. Levin and Z.-C. Gu, "Braiding statistics approach to symmetry-protected topological phases," Phys. Rev. B, vol. 86, p. 115109, Sep 2012.

[18] X. Chen, Z.-C. Gu, Z.-X. Liu, and X.-G. Wen, "Symmetry protected topological orders and the group cohomology of their symmetry group," Phys. Rev. B, vol. 87, p. 155114, Apr 2013.

[19] A. Kapustin, "Symmetry protected topological phases, anomalies, and cobordisms: beyond group cohomology," arXiv preprint arXiv:1403.1467, 2014.

[20] B. Yoshida, "Topological phases with generalized global symmetries," Phys. Rev. B, vol. 93, p. 155131, Apr 2016.

[21] B. Yoshida, "Topological color code and symmetry-protected topological phases," Phys. Rev. B, vol. 91, p. 245131, Jun 2015.

[22] B. Yoshida, "Gapped boundaries, group cohomology and fault-tolerant logical gates," Annals of Physics, vol. 377, pp. 387-413, 2017.

[23] M. A. Levin and X.-G. Wen, "String-net condensation: a physical mechanism for topological phases," Phys. Rev. B, vol. 71, p. 045110, Jan 2005.

[24] A. Y. Kitaev, "Fault tolerant quantum computation by anyons," Annals Phys., vol. 303, pp. 2-30, 2003.

[25] H. Bombin and M. A. Martin-Delgado, "Family of non-Abelian Kitaev models on a lattice: Topological condensation and confinement," prb, vol. 78, p. 115421, Sept. 2008. 
[26] O. Buerschaper and M. Aguado, "Mapping Kitaev's quantum double lattice models to Levin and Wen's string-net models," prb, vol. 80, p. 155136, Oct. 2009.

[27] A. Y. Kitaev, "Quantum computations: algorithms and error correction," Russian Mathematical Surveys, vol. 52, no. 6, p. 1191, 1997.

[28] C. Aneziris, A. P. Balachandran, L. Kauffman, and A. M. Srivastava, "Novel Statistics for Strings and String "CHERN-SIMON" Terms," International Journal of Modern Physics A, vol. 6, pp. 2519-2558, 1991.

[29] D. L. Goldsmith, "The theory of motion groups.," Michigan Math. J., vol. 28, no. 1, pp. 3-17, 1981.

[30] D. L. Goldsmith, "Motions of links in the 3-sphere," Bull. Amer. Math. Soc., vol. 80, pp. 62-66, 011974.

[31] C. Damiani, "A journey through loop braid groups," ArXiv e-prints, May 2016.

[32] Z. Kadar, P. Martin, E. Rowell, and Z. Wang, "Local representations of the loop braid group," ArXiv e-prints, Nov. 2014.

[33] P. Bruillard, L. Chang, S.-M. Hong, J. Y. Plavnik, E. C. Rowell, and M. Y. Sun, "Low-dimensional representations of the three component loop braid group," Journal of Mathematical Physics, vol. 56, p. 111707, Nov. 2015.

[34] G. Kuperberg, "Involutory Hopf algebras and 3-manifold invariants," ArXiv Mathematics e-prints, May 1992.

[35] V. Turaev and O. Viro, "State sum invariants of 3-manifolds and quantum 6jsymbols," Topology, vol. 31, no. 4, pp. 865 - 902, 1992.

[36] A. Davydov, "Modular invariants for group-theoretical modular data. i," Journal of Algebra, vol. 323, no. 5, pp. 1321 - 1348, 2010.

[37] J. Milnor and J. Stasheff, Characteristic Classes, by John W. Milnor and James D. Stasheff. Annals of Mathematics Studies, No. 76, 1974.

[38] C.-H. Lin and M. Levin, "Loop braiding statistics in exactly soluble 3D lattice models," ArXiv e-prints, Feb. 2015.

[39] R. Oeckl, Discrete Gauge Theory: From Lattices to TQFT. Imperial College Press, 2005.

[40] J. W. Barrett and B. W. Westbury, "Invariants of Piecewise-Linear 3-Manifolds," ArXiv High Energy Physics - Theory e-prints, Nov. 1993. 
[41] N. Y. Reshetikhin and V. G. Turaev, "Ribbon graphs and their invariants derived from quantum groups," Comm. Math. Phys., vol. 127, no. 1, pp. 1-26, 1990.

[42] M. Lackenby, "A polynomial upper bound on Reidemeister moves," ArXiv e-prints, Feb. 2013.

[43] X.-G. Wen, Quantum field theory of many-body systems: from the origin of sound to an origin of light and electrons. Oxford University Press on Demand, 2004.

[44] S. D. Sarma and A. Pinczuk, Perspectives in quantum hall effects: Novel quantum liquids in low-dimensional semiconductor structures. John Wiley \& Sons, 2008.

[45] P. Anderson, "The resonating valence bond state in la2cuo4 and superconductivity," 1987.

[46] X.-G. Wen, "Vacuum degeneracy of chiral spin states in compactified space," Physical Review B, vol. 40, no. 10, p. 7387, 1989.

[47] X.-G. Wen, "Topological orders in rigid states," International Journal of Modern Physics B, vol. 4, no. 02, pp. 239-271, 1990.

[48] C. Nayak, S. H. Simon, A. Stern, M. Freedman, and S. D. Sarma, "Non-abelian anyons and topological quantum computation," Reviews of Modern Physics, vol. 80, no. 3, p. 1083, 2008.

[49] M. Freedman, A. Kitaev, M. Larsen, and Z. Wang, "Topological quantum computation," Bulletin of the American Mathematical Society, vol. 40, no. 1, pp. 31-38, 2003.

[50] M. J. Bernabé Ferreira, P. Padmanabhan, and P. Teotonio-Sobrinho, "2D Quantum Double Models From a 3D Perspective," ArXiv e-prints, Oct. 2013.

[51] P. Padmanabhan, J. P. Ibieta Jimenez, M. J. Bernabé Ferreira, and P. TeotonioSobrinho, "More Solvable \$2D $\$$ Quantum Models from Lattice Gauge Theories," ArXiv e-prints, Aug. 2014.

[52] R. Brown, "Cohomology with chains as coefficients," Proc. London Math. Soc. (3), vol. 14, pp. 545-565, 1964.

[53] K. G. Wilson, "Confinement of quarks," Phys. Rev. D, vol. 10, pp. 2445-2459, Oct 1974.

[54] C. Nash and S. Sen, Topology and geometry for physicists. Academic Press, 1983.

[55] J. C. Baez and U. Schreiber, "Higher Gauge Theory," ArXiv Mathematics e-prints, Nov. 2005. 
[56] J. C. Baez and J. Huerta, "An invitation to higher gauge theory," General Relativity and Gravitation, vol. 43, pp. 2335-2392, Sept. 2011.

[57] A. Ashtekar and J. Lewandowski, "Differential geometry on the space of connections via graphs and projective limits," J. Geom. Phys., vol. 17, pp. 191-230, 1995.

[58] J. C. Baez and S. Sawin, "Functional integration on spaces of connections," 1995.

[59] J. Lewandowski and T. Thiemann, "Diffeomorphism invariant quantum field theories of connections in terms of webs," Class. Quant. Grav., vol. 16, pp. 2299-2322, 1999.

[60] J. H. C. Whitehead, "Combinatorial homotopy. I," Bull. Amer. Math. Soc., vol. 55, pp. 213-245, 1949.

[61] S. MacLane and J. H. C. Whitehead, "On the 3-type of a complex," Proc. Nat. Acad. Sci. U. S. A., vol. 36, pp. 41-48, 1950.

[62] J. C. Baez and A. D. Lauda, "Higher-dimensional algebra. V. 2-groups," Theory Appl. Categ., vol. 12, pp. 423-491, 2004.

[63] L. Crane, L. H. Kauffman, and D. Yetter, "Evaluating the Crane-Yetter invariant," 1993.

[64] L. Crane and D. Yetter, "A categorical construction of 4d topological quantum field theories," Quantum topology, vol. 3, 1993.

[65] F. Alexander Bais, P. van Driel, and M. de Wild Propitius, "Quantum symmetries in discrete gauge theories," Physics Letters B, vol. 280, pp. 63-70, Apr. 1992.

[66] R. Dijkgraaf, V. Pasquier, and P. Roche, "Quasi-quantum groups related to orbifold models," 1990.

[67] A. Kitaev and L. Kong, "Models for Gapped Boundaries and Domain Walls," Communications in Mathematical Physics, vol. 313, pp. 351-373, July 2012.

[68] G. James and M. Liebeck, Representations and Characters of Groups. Cambridge mathematical textbooks, Cambridge University Press, 2001.

[69] S. Beigi, P. W. Shor, and D. Whalen, "The Quantum Double Model with Boundary: Condensations and Symmetries," Communications in Mathematical Physics, vol. 306, pp. 663-694, Sept. 2011.

[70] P. Etingof, D. Nikshych, and V. Ostrik, "On fusion categories," ArXiv Mathematics e-prints, Mar. 2002. 
[71] E. Rowell, R. Stong, and Z. Wang, "On classification of modular tensor categories," ArXiv e-prints, Dec. 2007.

[72] J. Serre, Linear Representations of Finite Groups. Graduate texts in mathematics, Springer-Verlag, 1996.

[73] W. Fulton and J. Harris, Representation Theory: A First Course. Graduate Texts in Mathematics, Springer New York, 1991.

[74] M. Forrester-Barker, "Group Objects and Internal Categories," ArXiv Mathematics e-prints, Dec. 2002.

[75] A. Hatcher, Algebraic Topology. Cambridge University Press, 2002.

[76] A. Kitaev, "Anyons in an exactly solved model and beyond," Annals of Physics, vol. 321, pp. 2-111, Jan. 2006.

[77] C. W. von Keyserlingk, F. J. Burnell, and S. H. Simon, "Three-dimensional topological lattice models with surface anyons," Phys. Rev. B, vol. 87, p. 045107, Jan 2013.

[78] A. S. Crans and F. Wagemann, "Crossed modules of racks," ArXiv e-prints, Oct. 2013.

[79] M. Hastings, "Quantum codes from high-dimensional manifolds," arXiv preprint arXiv:1608.05089, 2016.

[80] A. R. Calderbank and P. W. Shor, "Good quantum error-correcting codes exist," Physical Review A, vol. 54, no. 2, p. 1098, 1996.

[81] S. J. Devitt, W. J. Munro, and K. Nemoto, "Quantum error correction for beginners," Reports on Progress in Physics, vol. 76, p. 076001, July 2013.

[82] H. Bombin and M. A. Martin-Delgado, "Homological error correction: Classical and quantum codes," Journal of Mathematical Physics, vol. 48, no. 5, p. 052105, 2007.

[83] H. Bombin, "An introduction to topological quantum codes," arXiv preprint arXiv:1311.0277, 2013.

[84] S. Bravyi and M. B. Hastings, "Homological product codes," in Proceedings of the 46th Annual ACM Symposium on Theory of Computing, pp. 273-282, ACM, 2014.

[85] S. B. Bravyi and A. Y. Kitaev, "Quantum codes on a lattice with boundary," arXiv preprint quant-ph/9811052, 1998. 
[86] P. Vrana and M. Farkas, "Homological codes and abelian anyons," arXiv preprint arXiv:1505.01001, 2015.

[87] J. T. Anderson, "Homological stabilizer codes," Annals of Physics, vol. 330, pp. 122,2013 .

[88] A. R. Calderbank and P. W. Shor, "Good quantum error-correcting codes exist," Phys. Rev. A, vol. 54, pp. 1098-1105, Aug. 1996.

[89] P. W. Shor, "Scheme for reducing decoherence in quantum computer memory," Phys. Rev. A, vol. 52, pp. R2493-R2496, Oct 1995.

[90] A. Steane, "Multiple-Particle Interference and Quantum Error Correction," Proceedings of the Royal Society of London Series A, vol. 452, pp. 2551-2577, Nov. 1996.

[91] A. Kapustin and R. Thorngren, "Higher symmetry and gapped phases of gauge theories," arXiv preprint arXiv:1309.4721, 2013.

[92] G. Naber, Topology, Geometry and Gauge fields: Foundations. Texts in Applied Mathematics, Springer, 2010.

[93] M. Nakahara, Geometry, Topology and Physics, Second Edition. Graduate student series in physics, Taylor \& Francis, 2003.

[94] S. Kobayashi and K. Nomizu, Foundations of Differential Geometry. No. v. 2 in A Wiley Publication in Applied Statistics, Wiley, 1996.

[95] M. Spivak, A comprehensive introduction to differential geometry. No. v. 2 in A Comprehensive Introduction to Differential Geometry, Publish or Perish, inc., 1979.

[96] R. E. Mø gelberg, "A natural classifying space for cohomology with coefficients in a finite chain complex," Topology Proc., vol. 28, no. 1, pp. 199-217, 2004. Spring Topology and Dynamical Systems Conference. 